\title{
A COMPARATIVE STUDY OF THE MECHANICAL PERFORMANCE OF PLA SPECIMENS MANUFACTURED USING COMPRESSION MOLDING AND 3D PRINTING.
}

\author{
by \\ Vikas Chandran \\ Bachelor of Engineering, Visvesvaraya Technological University (2011)
}

\author{
A project \\ presented to Ryerson University \\ in partial fulfilment of the \\ requirements for the degree of \\ Master of Engineering \\ in the program of \\ Aerospace Engineering
}

Toronto, Ontario, Canada, 2019

OCVikas Chandran 2019 


\section{AUTHOR'S DECLARATION FOR ELECTRONIC SUBMISSION OF A PROJECT}

I hereby declare that I am the sole author of this project. This is a true copy of the project, including any required final revisions, as accepted by my examiners.

I authorize Ryerson University to lend this project to other institutions or individuals for scholarly research.

I further authorize Ryerson University to reproduce this project by photocopying or by other means, in total or in part, at the request of other institutions or individuals for the purpose of scholarly research.

I understand that my project may be made electronically available to the public. 


\title{
A COMPARATIVE STUDY OF THE MECHANICAL PERFORMANCE OF PLA SPECIMENS MANUFACTURED USING COMPRESSION MOLDING AND 3D PRINTING.
}

\author{
Vikas Chandran \\ Master of Engineering, Aerospace Engineering, Ryerson University, Toronto (2019)
}

\begin{abstract}
Compression molding is known to be one of the most cost-effective method to manufacture Polymer-based composite parts including pure thermoplastics, e.g. PolyLactic Acid (PLA). One of the current research questions is how a new innovative technology like 3D printing compares to compression molding in terms of mechanical performance of final parts.

This study aims at comparing the mechanical performance of dog-bone tensile coupons manufacturing using compression molding and 3D printing. The compression molding manufacturing process parameters are optimized to obtain maximum mechanical properties. This study investigates the effect of each parameter, including processing temperature, processing pressure, and dwell times on tensile modulus and strength of a pure PLA part coupon per ASTM D638-14. The entire study is conducted using a total of 54 specimens manufactured in a set of 9 batches for various combination of process parameters per Design Of Experiment (DOE). Optimum process parameters for PLA 3D printing have been obtained from previous studies and are used for comparison purposes. A comparison of mechanical performance of ASTM D638 coupons manufactured using optimum compression molding and 3D printing techniques is performed.
\end{abstract}




\section{ACKNOWLEDGEMENTS}

First, I would like to thank my supervisors Dr. Habiba Bougherara of the Department of Mechanical and Industrial Engineering and Dr. Kazem Fayazbakhsh of the Department of Aerospace Engineering at Ryerson University. Their valued guidance and support helped me advance my knowledge and understanding in the field of Compression molding, thermoplastics, testing standards and advanced composite materials. Their continuous feedbacks have been very significant in steering my project work towards the right direction.

I thank my fellow graduate students Ahmed Sarwar, Zainab Al-Hajaj and Wilfred Ekeoseye for their assistance and thoughts that helped me better execute this project. Likewise, I would like to extent my gratitude to Mr. Chris Chu and Mr. Allan Machin for their contribution during the manufacturing and testing phases of my project.

Finally, I would like to thank my family for their understanding, support and encouragement throughout the course of this project. 
TABLE OF CONTENTS

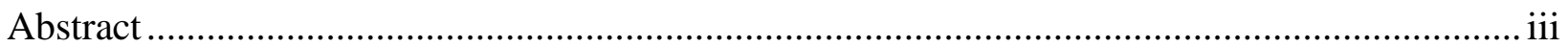

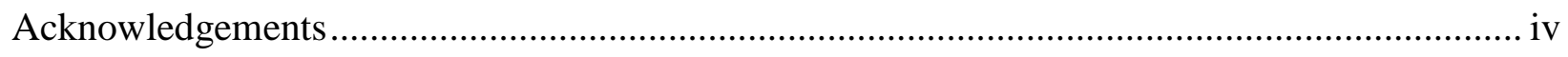

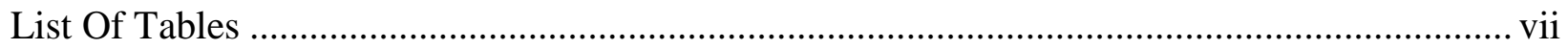

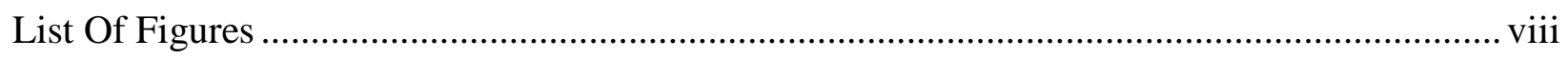

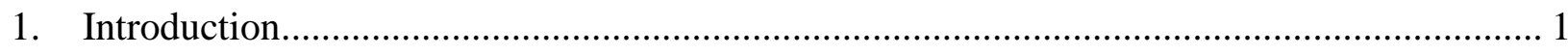

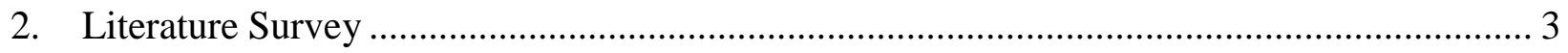

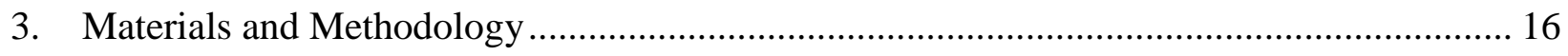

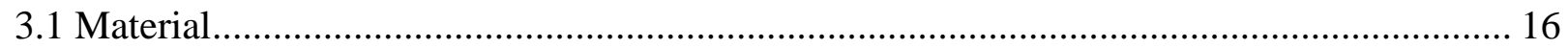

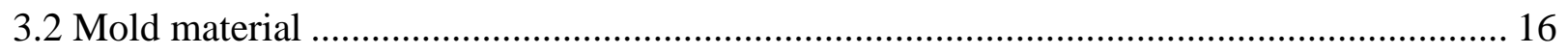

3.3 Compression Molding Machine ……………………..................................................... 17

3.4 Compression molding procedure .............................................................................. 20

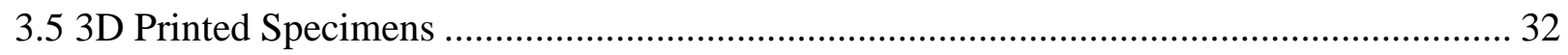

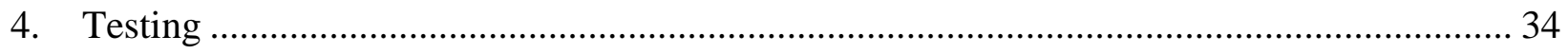

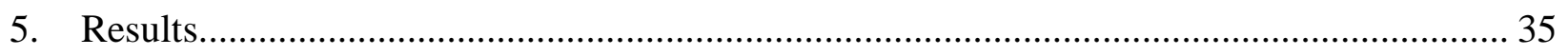

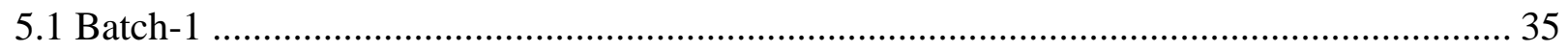

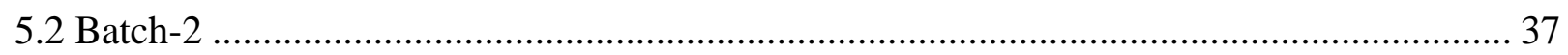

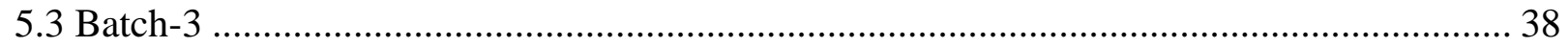

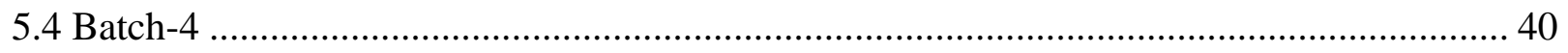


5.6 Batch-6 43

5.7 Batch-7 44

5.8 Batch-8 46

5.9 Batch-9 48

6. Discussion. 52

7. Comparison with 3D printed PLA specimens 54

8. Conclusion and Future Scope 59

9. References. 


\section{LIST OF TABLES}

Table 1: Strength of various composites showing the effect of fiber orientation [9]................. 7

Table 2: A summary of the literature review investigating the impact of process parameters on

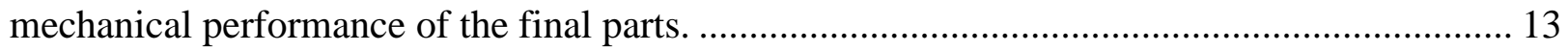

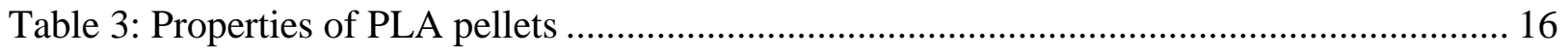

Table 4: Manufacturing Parameters of Compression molding in this study........................... 19

Table 5: Test plan for different Processing temperatures, Pressure and Dwell times............... 20

Table 6: Pressure applied in the study for compression molding ........................................ 22

Table 18: Manufacturing and design parameters for specimen 3D printing ........................... 33

Table 7: Tensile strength, modulus and failure strain for Batch-1 specimens. ........................ 35

Table 8: Tensile strength, modulus and failure strain for Batch-2 specimens ......................... 37

Table 9: Tensile strength, modulus and failure strain for Batch-3 specimens ........................ 38

Table 10: Tensile strength, modulus and failure strain for Batch-4 specimens. ....................... 40

Table 11: Tensile strength, modulus and failure strain for Batch-5 specimens. ..................... 41

Table 12: Tensile strength, modulus and failure strain for Batch-6 specimens. ...................... 43

Table 13: Tensile strength, modulus and failure strain for Batch-7 specimens. ...................... 44

Table 14: Tensile strength, modulus and failure strain for Batch-8 specimens. ....................... 46

Table 15: Tensile strength, modulus and failure strain for Batch-9 specimens. ...................... 48

Table 16: Averaged Tensile strength, Modulus and failure strain values for all batches .......... 49

Table 17: Weighted average property (WAP) comparison of all batches............................... 53

Table 19: Tensile strength, tensile modulus and failure strain of 3D printed specimens........... 54

Table 20: Comparison of Compression molded PLA specimen and 3D printed PLA specimen. 56

Table 21: Weighted average property comparison of compression molded specimens and 3D

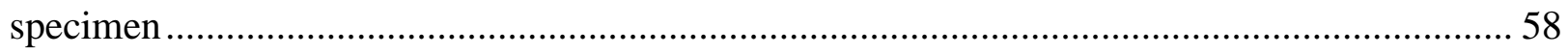




\section{LIST OF FIGURES}

Figure 1: Various synthesis methods of PLA [1] ........................................................... 3

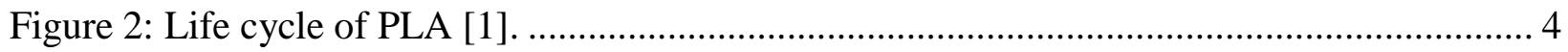

Figure 3: Drawing of the $12 \times 12$ in. mold with eleven coupon cavities: (a) Front view; (b) Side

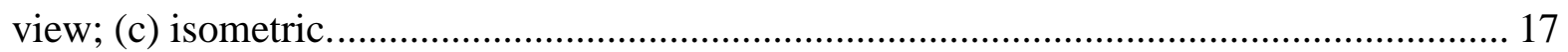

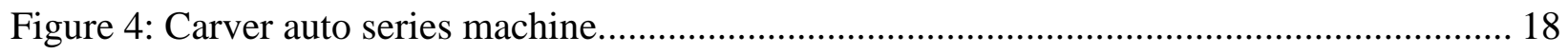

Figure 5: PLA pellets in mold cavity......................................................................... 21

Figure 6: Picture showing the mold containing the PLA pellets in the machine....................... 21

Figure 7: The mold with the metal tabs. ........................................................................... 22

Figure 8: Batch-1: a) temperature and pressure cycle; b) Fabricated Batch-1 specimens after

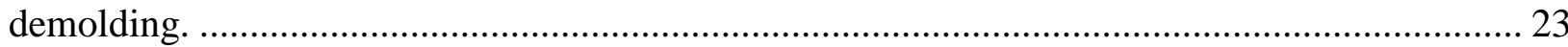

Figure 9: Batch-2: a) temperature and pressure cycle; b) Fabricated Batch-2 specimens after

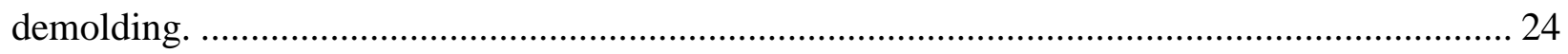

Figure 10: Batch-3: a) temperature and pressure cycle; b) Fabricated Batch-3 specimens after

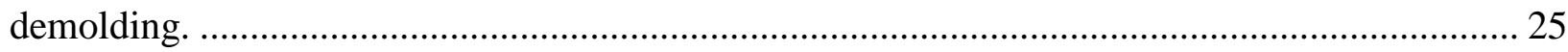

Figure 11: Batch-4: a) temperature cycle and pressure cycle; b) Fabricated batch- 4 specimens after

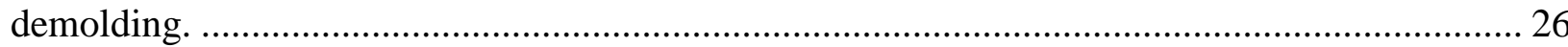

Figure 12: Batch-5: a) temperature cycle, and pressure cycle; b) Fabricated batch-5 specimens after

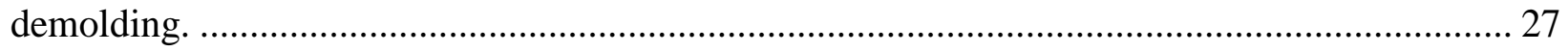
Figure 13: Batch-6: a) temperature cycle, and pressure cycle; b) Fabricated batch-6 specimens after demolding. 28

Figure 14: Batch-7: a) temperature cycle, and pressure cycle; b) Fabricated batch-7 specimens after demolding. .....

Figure 15: Batch-8: a) temperature cycle, and pressure cycle; b) Fabricated batch-8 specimens after demolding. ..... 30

Figure 16: Batch-9: a) temperature cycle, and pressure cycle; b) Fabricated batch-9 specimens after

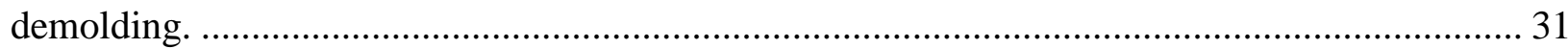

Figure 17: Materials deposition visualization in Simplify3D for $0^{\circ}$ layer-specimen................. 32

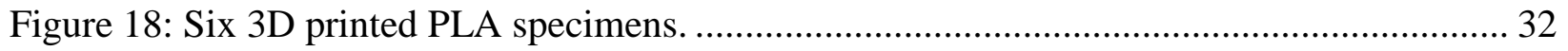

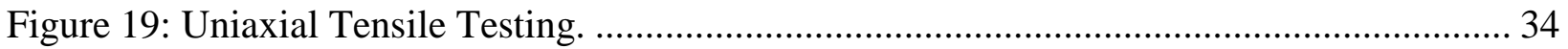

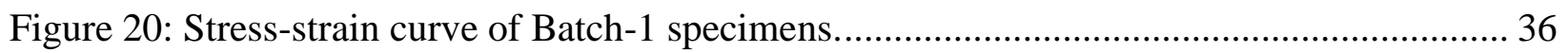


Figure 21: Fractured specimens of Batch-1 after tensile test............................................... 36

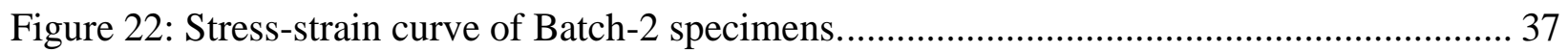

Figure 23: Fractured specimens of Batch-2 after tensile test............................................. 38

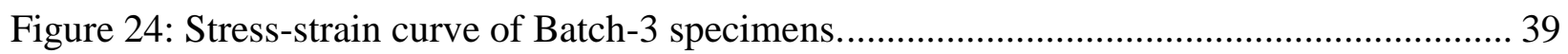

Figure 25: Fractured specimens of Batch-3 after tensile test................................................ 39

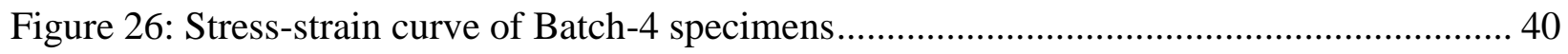

Figure 27: Fractured specimens of Batch-4 after tensile test.............................................. 41

Figure 28: Stress-strain curve of Batch-5 specimens...................................................... 42

Figure 29: Fractured specimens of Batch-5 after tensile test.............................................. 42

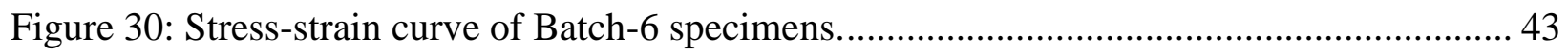

Figure 31: Fractured specimens of Batch- 6 after tensile test................................................ 44

Figure 32: Stress-strain curve of Batch-7 specimens....................................................... 45

Figure 33: Fractured specimens of Batch-7 after tensile test............................................ 45

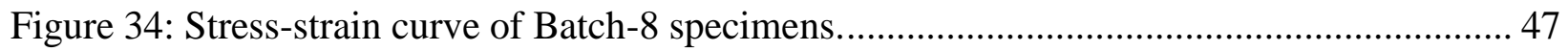

Figure 35: Fractured specimens of Batch-8 after tensile test............................................ 47

Figure 36: Stress-strain curve of Batch-9 specimens....................................................... 48

Figure 37: Fractured specimens of Batch-9 after tensile test............................................ 49

Figure 38: Comparison of averaged tensile strength values for all batches. ........................... 50

Figure 39: Averaged tensile modulus comparison of all batches. ......................................... 51

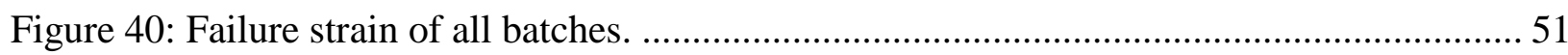

Figure 41: Stress- Strain graph of 3D printed specimens. ............................................... 55

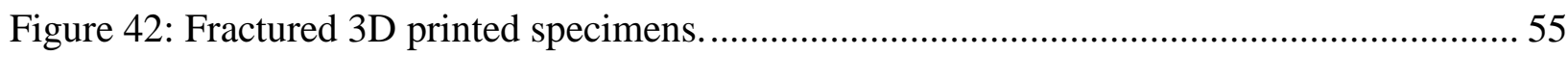

Figure 43 : Stress- Strain curve comparison of CM specimen vs 3D specimen........................ 57 


\section{NOMENCLATURE}

PBS Polybutylene succinate

PLA Polylactic acid

PHB Polyhydroxy Butyrate

$\mathrm{CM}$ Compression molding

DT Dwell Time

FDM Fused deposition modelling

FFF Fused filament fabrication

IM Injection Molding

$\mathrm{V}_{\mathrm{f}} \quad$ Fiber volume fraction

$\mathrm{Wt} \%$ Weight percentage of fiber 


\section{INTRODUCTION}

The fundamental change in dependence on conventional materials have been in motion for over a decade now, and the focus have been the new-generation green/bio composites with both natural fibers and a biodegradable matrix system. The emphasis for non-toxic, biodegradable materials for everyday packaging requirement in food industry to structural stability in Aerospace industry has risen due to the lack of reuse and recyclability of the current polymer-based composites. Unsurprisingly, using green composites takes the burden from the petro-based plastics for manufacturing composites.

Bio composites reinforced with natural fibers have shown a good improvement in mechanical properties and this is partially credited to the use of polymers as matrix. Besides fibers in a composite, the next obvious and vital constituent is the matrix material. A good matrix material improves the strength of the composite by transferring loads to the fibers and holding them together, which gives the composite its distinct mechanical properties. Polymer based composites besides being eco-friendly have definite edge over metal-based composites for being light weight, have lower cost of production and reduced manufacturing time. Several polymers that are currently used in industry are Polyethylene, epoxies, and Polypropylene which are not entirely biodegradable and have led to the need for bio-polymers like PLA, polyhydroxy butyrate (PHB), etc. [1].

Among thermoplastics, PolyLactic Acid (PLA) has gained great attention due to its high strength and high modulus properties as well as the ease of processing to make composites using conventional methods such as compression molding, injection molding or even additive manufacturing. The popularity of PLA when compared to other thermoplastics like Polypropylene and Polyamide (PA 12), credited to the ease of production, which is done by synthesizing the lactic acid of renewable resources through a few biological processes. In addition, the flexibility to make changes to the manufacturing process to obtain various molecular weights depending on application has made it more desirable [1]. PLA is thermally stable and have low melting temperature which makes it easier for composite fabrication with natural fibers, as this will not result in fiber strength degradation. Furthermore, the degradation of PLA requires no catalyst or enzymes as it is purely made of ester bonds that degrade with time; therefore, PLA is environmentally friendly and safe. 
Composite manufacturing methodologies greatly influence the mechanical property that PLA imparts to the composite. Several studies investigated polymer composites manufactured through conventional methods, like compression molding, injection molding, hand layup, etc. Compression molding unlike other conventional process produces uniform and isotropic components and has advantage of lower production cost and shorter manufacturing period. Pure PLA polymer and PLA based composites tend to have greater mechanical properties when manufactured by compression molding. Also, there is a certain degree of liberty to customize large parts as per requirement when compared to injection molding and Fused Deposition Modelling (FDM) methods.

Many researchers explored how different fibers strengthen PLA based composites and how process parameters impacted them. However, the study of pure PLA and the influence of several parameters on its strength is not yet investigated. This project focuses on this gap and the lack of research on this topic by investigating how several manufacturing factors like temperature, pressure, and dwell time influence the properties of pure PLA when manufactured by compression molding. In addition, a comparative study of the mechanical properties of compression molded PLA parts against 3D printed PLA parts is another focus of this research project. 


\section{LITERATURE SURVEY}

\section{PLA: Synthesis and life cycle of a Biopolymer}

PLA exists in semi-crystalline or amorphous structure, depending on the method of synthesizing the lactic acid from bacterial fermentation process of starch and polysaccharides, which are abundant in agricultural products like corn, soya, sugar cane and various other bio masses. In addition to biological methods, lactic acids are produced by chemical processes too. Lactic acid exists in terms of two active monomer configurations D-enantiomers and L-enantiomers and polymers obtained from both configurations are generally denoted as PLA [1].

Polymerization of Lactic acid can be performed in four methods which dictates the type of PLA, PLA with either high molecular weight or low molecular weight, that are produced as shown in Figure 1.

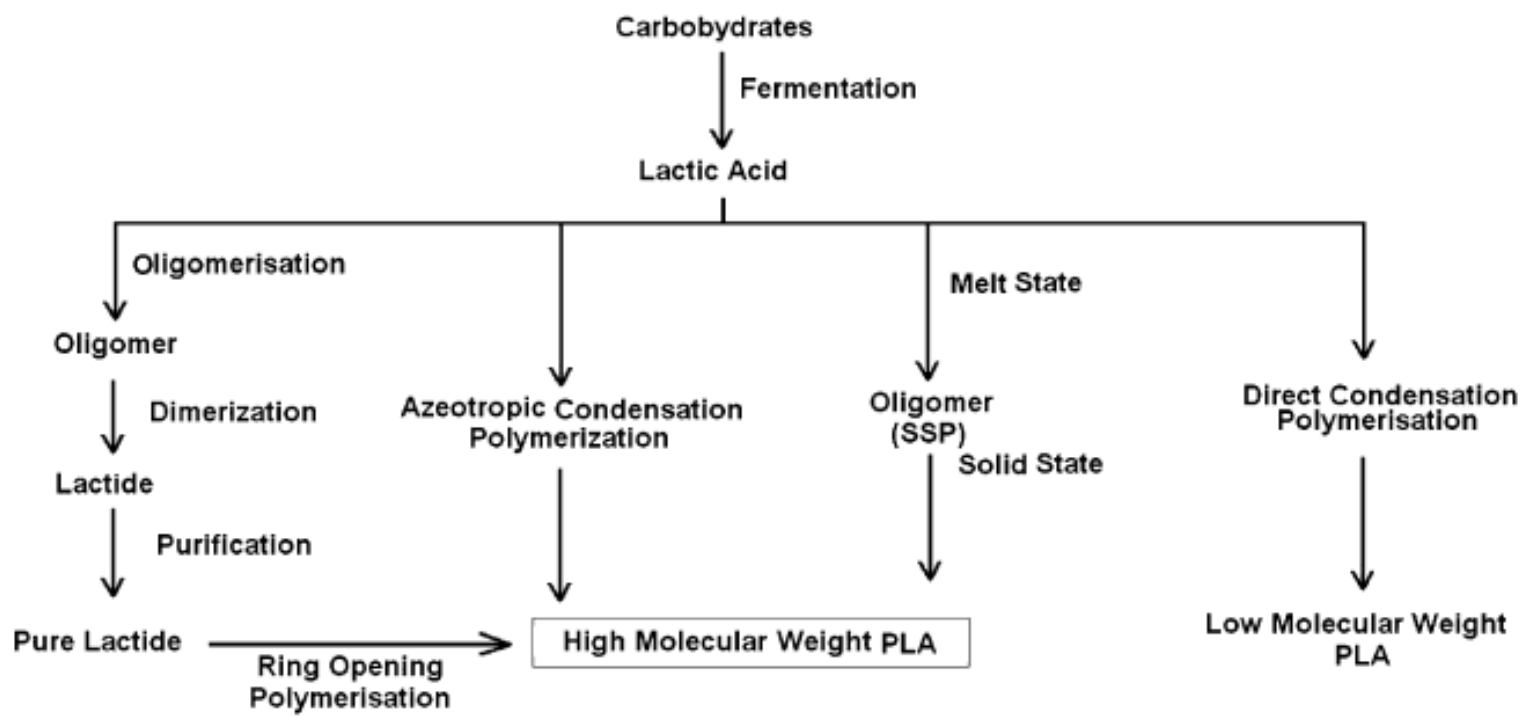

Figure 1: Various synthesis methods of PLA [1].

Mechanical properties of PLA vary depending on the molecular weight ranging from soft, elastic plastic to stiff and high strength plastic. In biomedical application, PLA for orthopedic implants requires higher molecular weight since the implants needs to have high strength. For pharmaceutical applications, where PLA is used as carrier for drugs, it is required that it has slower degradation time, thus lower molecular weight PLA molecules are necessary. This contrasting 
demand are met by modifying the synthesis process of lactic acid by either condensation or ring opening polymerization of cyclic lactide.

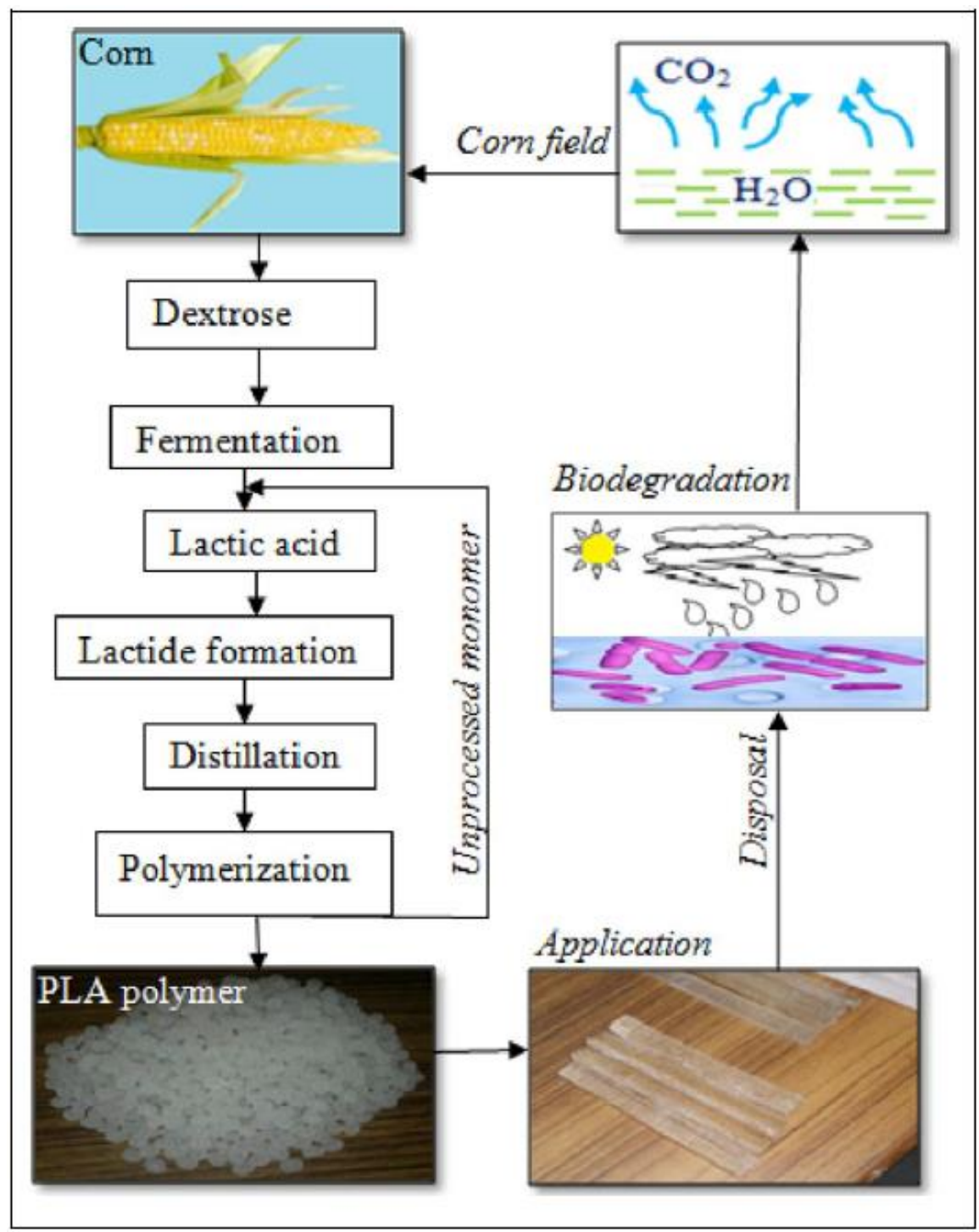

Figure 2: Life cycle of PLA [1].

Biodegradation of PLA is ensured solely by bio organism that breaks it to non-toxic metabolites by hydrolysis of the ester groups to water soluble oligomers and monomers. The bi-products of this degradation is just water and carbon dioxide that goes back into nourishing the biomass that PLA originated from. This degradation process is dependent on time, temperature and $\mathrm{pH}$ content in the environment. Lack of controllability in the degradation process is a drawback, and to 
overcome this drawback, research suggests manufacturing several blends of PLA in combination with other bio polymers without decreasing the mechanical property of already brittle PLA [1].

\section{PLA- Green composites}

Composites based on PLA, as the matrix, has found a foot hold across industries owing to its unique strength properties and ease of processing. PLA-based composites can be mass-produced by conventional methods like hand layup, tape layup, compression molding, transfer molding, and injection molding, or by the relatively new methods like FDM. Among the bio-degradable polyesters that are presently used in engineering industry, PLA possess better mechanical properties and this is demonstrated by Shibata et al. [2] by comparing polymers Poly(butylene succinate) PBS, poly(3-hydroxybutyrate-co-3-hydroxyvarelate) PHBV prepared by compression molding. Depending on their melting temperature, polymers were processed for different temperatures like 160, 170 and $160{ }^{\circ} \mathrm{C}$ for PHBV, PBS and PLA, respectively and for a uniform pressure of $3 \mathrm{MPa}$. Tensile strength and modulus of pure PHBV specimens were $16.3 \mathrm{MPa}$ and 0.44GPa, respectively. Likewise, PBS specimens showed tensile strength and modulus of 37.6 $\mathrm{MPa}$ and 0.63GPa, respectively. While, PLA specimens showed 45.2MPa and 2.82GPa of tensile strength and modulus, respectively. PLA based composites has advantage of the strength and property that PLA imparts to the composites, however this largely depend on various aspects and conditions like:

\section{$>$ Type of Fiber as reinforcement}

○ Fiber weight (wt.\%)

- Fiber length

$\bigcirc$ Fiber surface treatment

\section{Manufacturing process employed,}

Processing conditions- Temperature, Pressure and Time 


\section{Fiber type}

Strength and properties of PLA are improved when reinforced with fibers. Application of natural fibers have numerous advantages such as environmentally friendly, offers an alternate solution to the conventional reinforcing fibers, inexpensive, low density and good specific strength. The most desired properties in a natural fiber are the higher cellulose content and a low micro fibril angle [3]. Despite these advantages, natural fibers have certain limitations like fluctuations of fiber fineness and strength along the length of the fiber, difficult to impregnate with a thermoplastic resin and lower mechanical property when compared to synthetic fibers [3]. Strength of a green composite is determined by the type of natural fiber used, fiber content (wt.\%) used and the how well the fiber is impregnated by the resin which governs the interfacial property and how effective is the load transfer between the fiber and the matrix.

Graupner et al. [9] illustrated a comprehensive study of PLA based composites made of natural fibers such as Jute, Cotton, Hemp, Kenaf, and Lyocell comparing strength and properties in both $0^{\circ}$ and $90^{\circ}$. Samples were fabricated by compression molding with pressure of $4.2 \mathrm{MPa}$ at $180{ }^{\circ} \mathrm{C}$ for 20mins. For $0^{\circ}$, highest tensile strength was reported for Lyocell/PLA and Hemp/PLA with 81.8 $\pm 6.6 \mathrm{MPa}$ and 57.5 $\pm 3.6 \mathrm{MPa}$, respectively, followed by Kenaf/PLA and Cotton/PLA with $52.9 \pm 4.5 \mathrm{MPa}$ and 41.2 $\pm 2 \mathrm{MPa}$, respectively. All $0^{\circ}$ fiber reinforced PLA composite exceeded tensile strength of pure PLA sample's tensile strength of $30.1 \pm 8 \mathrm{MPa}$, however this is not true when fibers were oriented $90^{\circ}$. Except for Lyocell/PLA with tensile strength of $31.9 \pm 6 \mathrm{MPa}$ every other composite had lower values than pure PLA sample. To improve the strength in transverse direction, author suggests mixing of fibers, Lyocell 20\% + hemp 20\% and hemp 20\% + kenaf $20 \%$, which yielded better values of $41.4 \pm 9.8 \mathrm{MPa}$ and $34.4 \pm 5.8 \mathrm{MPa}$, respectively. 
Table 1: Strength of various composites showing the effect of fiber orientation [9].

\begin{tabular}{|c|c|c|c|c|}
\hline & \multirow{2}{*}{$\begin{array}{c}\text { Fiber } \\
\text { Orientation }\end{array}$} & \multicolumn{2}{|c|}{ Tensile Strength } & \multirow{2}{*}{$\begin{array}{c}\text { Young's Modulus } \\
\text { (MPa) }\end{array}$} \\
\hline & & $\begin{array}{c}\mathbf{n} \\
\text { (sample count) }\end{array}$ & MPa & \\
\hline Pure PLA sample & - & 10 & $30.1( \pm 8.00)$ & $3820.2( \pm 166.3)$ \\
\hline \multirow[t]{2}{*}{ Cotton-PLA } & $0^{\circ}$ & 6 & $41.2( \pm 2.0)$ & $4242.3( \pm 635.0)$ \\
\hline & $90^{\circ}$ & 5 & $26.1( \pm 3.3)$ & $3490.0( \pm 541.0)$ \\
\hline \multirow[t]{2}{*}{ Kenaf-PLA } & $0^{\circ}$ & 9 & $52.9( \pm 4.5)$ & $7138.6( \pm 1329.9)$ \\
\hline & $90^{\circ}$ & 5 & $24.1( \pm 1.3)$ & $4248.7( \pm 322.8)$ \\
\hline \multirow[t]{2}{*}{ Hemp-PLA } & $0^{\circ}$ & 8 & $57.5( \pm 3.6)$ & $8064.2( \pm 1125.4)$ \\
\hline & $90^{\circ}$ & 5 & $28.9( \pm 1.5)$ & $4238.6( \pm 523.0)$ \\
\hline \multirow[t]{2}{*}{ Lyocell-PLA } & $0^{\circ}$ & 6 & $81.8( \pm 6.6)$ & $6783.8( \pm 522.9)$ \\
\hline & $90^{\circ}$ & 5 & $31.9( \pm 6.0)$ & $4720.7( \pm 529.1)$ \\
\hline \multirow[t]{2}{*}{ Hemp/kenaf-PLA } & $0^{\circ}$ & 6 & $61.0( \pm 7.3)$ & $7763.8( \pm 354.0)$ \\
\hline & $90^{\circ}$ & 6 & $34.4( \pm 5.8)$ & $4920.8( \pm 661.5)$ \\
\hline \multirow{2}{*}{$\begin{array}{c}\text { Hemp/Lyocell- } \\
\text { PLA }\end{array}$} & $0^{\circ}$ & 11 & $71.5( \pm 8.7)$ & $7034.9( \pm 1037.0)$ \\
\hline & $90^{\circ}$ & 11 & $41.4( \pm 9.8)$ & $4642.9( \pm 711.2)$ \\
\hline
\end{tabular}

Results shown above assert how mechanical properties and strength of PLA based composites vary with the different types of natural fibers and the fact that combining multiple fibers with can yield improvement in strength properties in transverse direction. Another study with Ramie fiber and Jute fiber for different fiber content in a PLA based composite shows that beyond certain fiber weight percentage, the addition of fibers can weaken the strength rather than improving [16]. Ramie/PLA and Jute /PLA composite, with different fiber contents in the range of $10 \%$ to $50 \%$ manufactured by compression molding with $20 \mathrm{MPa}$ pressure at 4 mins and $170{ }^{\circ} \mathrm{C}$ temperature, was subjected to testing. In the case of Ramie/PLA, with 10, 20 and 30\% fiber content the tensile strength obtained was 50.51 and 53MPa which was better than that of pure PLA, 45MPa. However, with $40 \%$ and $50 \%$ fiber content, the strength was reduced to 46 and $44 \mathrm{MPa}$, respectively. Similar results recorded for Jute/PLA, 47, 49 and $50 \mathrm{MPa}$ for 10, 20 and 30\% fiber respectively, and a strength reduction to 47 and $43 \mathrm{MPa}$ for $40 \%$ and $50 \%$. This variation of strength was also observed 
in flexural and bending strengths of these composites. Another comparable study of Kenaf/PLA [15] ,for three different fiber wt.\% content, i.e. 30\%, 50\% and 70\% reported tensile strengths of 130.5, 210.9 and 223.3MPa respectively against the theoretical value calculated by rule of mixture 178.2, 297 and 415.8MPa respectively. Strength values reported for $70 \%$ fiber wt.\% was half of the theoretical value which states that fiber weight must be below $70 \%$ for fabricating Kenaf/PLA composites. This reduction is attributed to be caused due to insufficient resin content in the composite, which results in poor dissipation of fibers, consecutively results in formation of voids.

An alternate method to improve the strength of natural fiber reinforced composites without compromising the resin content is by heat treating the fibers [4]. Luffa/PLA composite with heat treated $15 \%$ luffa fiber reported tensile strength of 40.2 MPa against untreated luffa fiber for same fiber weight of only $35 \mathrm{MPa}$. This is because heat treatment of fibers partially removes hemicellulose that reduces the hydrophilic nature of the fiber, resulting in improved fiber-matrix bonding strength. Significant improvement in strength properties are observed with better interfacial adhesion property between the matrix and the fiber. Another comparative study of weaved fibers [5], suggests that adhesion can be improved by employing weaved fibers. This was verified with basket weave flax fiber and plain weave fibers. Results shows basket weave flax/PLA composite had better tensile strength as the area available for fiber matrix adhesion increased resulting in stronger composite. The interfacial adhesion and impregnation of fibers facilitates load transfer from matrix to the more load capable fibers.

Strength and properties of a composite are determined by the grade of consolidation between the fibers and the matrix. The higher the wettability of the fibers by the matrix, the stronger is the bond between the fiber and the matrix. Which will result in better capability of load transfer to the fibers. Generally, PLA is available as pellets or in amorphous forms. However, there are studies of PLA in fiber forms, sheet forms and, fabric forms to improve impregnability, reduce voids and eventually the composite strength. Weaving PLA fibers with Jute fiber was performed by Memon et al. [6].Winding the woven yarns of both PLA and Jute fibers on a metallic frame and then placing it on a heated mold then compression mold was at temperatures ranging from $185{ }^{\circ} \mathrm{C}$ to $235{ }^{\circ} \mathrm{C}$ and at a constant pressure of 1.33 MPa were used. They defined an achievement ratio which is calculated by normalizing the experimental value with theoretical value (rule of mixture). The achievement ratio of tensile modulus of the composite improved until $195^{\circ} \mathrm{C}$ as the viscosity 
reduced resulting better impregnation, but strength reduced on further increasing the temperature. This showed that although the tensile strength and elastic modulus improved with an increase in processing temperature because of a better impregnation, beyond $195^{\circ} \mathrm{C}$ Jute fibers deteriorated resulting in lower strength.

Another comparable study of PLA /Jute spun yarn on fiber impregnation in a PLA composite, and how surface treatment of the fibers influence was performed in [8] by 'micro-braiding' the fibers together. Braiding resin fibers alongside the reinforcement fiber was termed as 'Micro-braided' yarn, this way impregnation of the fiber was excellent since resin was closely placed with fiber. Jute fibers used were spun into a yarn to reduce the variability in individual fiber fineness, length and strength. These micro-braided fibers were then treated with various concentrations of shellac resin, from $0.2 \mathrm{wt} \%$ to $5 \mathrm{wt} \%$.

Influence of surface treatment was performed by compression molding at a pressure of $1.33 \mathrm{MPa}$ for a molding time of 8 mins at a temperature of $190{ }^{\circ} \mathrm{C}$. The achievement ratios of both tensile strength and elastic modulus were increased until shellac concentration of $2.0 \mathrm{wt} \%$ and began to reduce significantly for $5.0 \mathrm{wt} \%$. The resin adhesion is negligible for concentration below $0.5 \mathrm{wt} \%$ and was improved for $2.0 \mathrm{wt} \%$, an increase of $90 \%$ in the tensile strength. It was confirmed that for $5.0 \mathrm{wt} \%$ of shellac the resin was not able to penetrate the fiber and thus the impregnation was poor. This verifies that fiber treatment with shellac resin improves the tensile properties by improving the interfacial property; however, this is not the case if the concentration is too high.

\section{Manufacturing process- PLA composites}

Conventional methods like Compression molding and injection molding are very popular for manufacturing polymer-based composites. Manufacturing process in both methodologies applies a combination of pressure and temperature to fabricate composites with better mechanical properties. Investigations of newer manufacturing technique like FDM shows potential for competing with conventional methods in terms of ease of use and minimum human interface in the process. However, processes like FDM have been limited to making of prototypes and mockups because of inability to produce products for mass production. The mechanical performance of 3D printed parts depends on manufacturing process and design parameters, e.g. nozzle temperature, bed temperature, printing speed, raster angle, build orientation, layer thickness, etc. 
These parameters can be optimized to achieve maximum improvement in tensile strength and modulus.

Carneiro et al. [12] addressed how FDM has the potential of competing with conventional methods by comparing the mechanical properties of a glass fiber reinforced polypropylene composite manufactured by compression molding and FDM method. Tensile modulus and strength of pure polypropylene manufactured by compression molding were reported as $1.5 \mathrm{GPa}$ and $42 \mathrm{MPa}$, respectively against $1.0 \mathrm{GPa}$ and $27 \mathrm{MPa}$ of tensile modulus and strength respectively by FDM. Same trend was observed in the results of glass reinforced polypropylene (GRPP) composite where compression molded coupons obtained tensile modulus of $2.2 \mathrm{GPa}$ and tensile strength of $55 \mathrm{MPa}$ in comparison to composites from FDM with $1.4 \mathrm{GPa}$ and $40 \mathrm{MPa}$ of tensile modulus and strength, respectively. The loss of mechanical strength reported for printed samples is in the range of 20$30 \%$, and author concludes that further investigations are required to make this process efficient to compete with the conventional methods.

Assessment among compression molding and injection molding have been documented in numerous researches but very few documents its impact in a PLA based natural fiber reinforced polymer composites. Srebrenkoska et al. [3] performed an investigation on how compression molding and injection molding methodologies influence a PLA based composites with Kenaf fibers and Rice hull fibers for different levels of temperature and pressure and their strength values. Factors influencing in a compression molding procedure are only molding temperature, pressure, and the dwell time, whereas in an injection molding system several factors like temperatures in the hopper, feeding zone, injecting nozzle along with pressure in the nozzle, compressing zone and packing stages influence the properties of the component manufactured. On comparison of the physical and mechanical properties of composites from both methods i.e. compression molded Kenaf /PLA and Ricehull/PLA at a temperature of $175{ }^{\circ} \mathrm{C}$ and a pressure of $15 \mathrm{MPa}$, exhibited tensile strength of $28.3 \pm 6.54 \mathrm{MPa}$ and $26.7 \pm 1.49 \mathrm{MPa}$, respectively. On the other hand, injection molded Kenaf/PLA and Ricehull/PLA had a strength of 21.8 \pm 1.02 and 20.6 $\pm 0.91 \mathrm{MPa}$ respectively. This clearly shows that there is a $25 \%$ reduction of strength when parts are injection molded than compression molded, [3]. The same trend was observed for flexural and compression strengths of parts.

A similar study documented by Graupner et al. [10] on lyocell/PLA composite's tensile and impact strength when fabricated by Compression Molding (CM) and Injection Molding (IM). Pure PLA 
fabricated by $\mathrm{CM}$ had a tensile strength of 51.9 MPa and IM had 59.9 MPa; however, results were reversed when PLA was reinforced with lyocell fibers. Composite with fiber content of $40 \%$ by weight from CM reported strength in the range of 92-118 $\mathrm{MPa}$, whereas IM composites had a strength in the range of 45-66 MPa. Even though CM composite may have more voids and gaps because of degassing and evaporation, variance in strength was investigated to be the lack in provision to control fiber alignment in an injection molded composite along with shorter fiber length when compared to composite manufactured from compression molding. Study emphasized that just ensuring that length of fibers is barely greater than critical length, $L>L_{c}$, is not enough instead fibers must be reasonably long enough for proper load transfer from matrix. This limitation of employing only shorter fibers in injection molding gives compression molding an edge. These results states that there is a great influence of processing technique on the mechanical strength of the fabricated composites and compression molding is ideal for manufacturing, especially when a thermoplastic resin system is used.

\section{Processing conditions: Temperature and Pressure}

Generally PLA have melting temperature in the range of $150-160{ }^{\circ} \mathrm{C}$ depending in the grade of PLA and glass transition temperature of PLA lies in the range of $60{ }^{\circ} \mathrm{C}$ which is higher compared to other polymers like PBS $\left(20-30{ }^{\circ} \mathrm{C}\right)$ and PHBV $\left(-32^{\circ} \mathrm{C}\right)$ therefore crystallization of PLA at room temperature is very slow [2]. Mechanical strength of PLA based composites manufactured by compression molding are characterized by the processing temperature, processing pressure, and the duration of pressure applied. Influence of process condition demonstrated by Kakar et al. [4] for various combination of processing temperature ranging between $150^{\circ} \mathrm{C}$ to $170^{\circ} \mathrm{C}$ and dwell time between 15 to 45 minutes for a constant $5 \mathrm{MPa}$ pressure shows how strength and properties are dependent of processing conditions. The optimized process parameters arrived after several iterations were compression molding pressure of $5 \mathrm{MPa}$ with temperature of $160^{\circ} \mathrm{C}$ and for a dwell time of 30 minutes. Pure PLA dog-bone sample fabricated with these parameters yielded a tensile strength of $46.8 \mathrm{MPa}$ this is due to complete melting of the PLA pellets and better compaction resulting in no voids. It is crucial to have processing temperature to be at either the melting temperature of PLA or for the duration long enough to ensure melting of PLA pellets and yet crucial to not over heat causing evaporation and subsequent bubble entrapment resulting voids. 
Similar study on effects of processing pressure, carried out by Rubio et al. [1] on the mechanical properties of PLA based flax composite for over a range of $8 \mathrm{MPa}$ and $32 \mathrm{MPa}$ pressure and constant temperature of $185{ }^{\circ} \mathrm{C}$ showed that the strength increased up to $8 \mathrm{MPa}$ of pressure and reduced beyond $32 \mathrm{MPa}$ that can be related to the fiber damage as result of high pressure. Processing temperature of $185^{\circ} \mathrm{C}$ was attained after observing fiber damages caused due to temperatures beyond $200{ }^{\circ} \mathrm{C}$ and incomplete melting of PLA pellets below $175^{\circ} \mathrm{C}$. Importance of manufacturing composites with right parameters of temperature and pressure is absolute necessary to ensure that both fiber and PLA retain their strength and properties. Outlining the exact temperature and pressure for each kind of natural fiber is vital and this is defined in Ochi [15] for Kenaf fibers in a compression molded Kenaf/PLA composite. To establish the ideal molding temperature for kenaf fibers, mechanical properties of Kenaf fibers at various temperature ranges of 160,180 , and $200^{\circ} \mathrm{C}$ were considered and for a duration of 15,30 , and 60 minutes, respectively. Tensile strength of Kenaf fiber decreased significantly when it was heated to $200{ }^{\circ} \mathrm{C}$ but remained unchanged at $180^{\circ} \mathrm{C}$ for 30 mins and at $160^{\circ} \mathrm{C}$ for longer durations. This confirms that processing temperature for Kenaf fiber for retaining integrity must be between 160 and $180{ }^{\circ} \mathrm{C}$, depending on the melting temperature of PLA grade used and for any duration above 30 mins the temperature should be maintained at $160^{\circ} \mathrm{C}$. Processing temperature also influences the interfacial adhesion of fiber and matrix, by reduced viscosity of PLA the fiber impregnation improves and making it possible to accommodate more fiber volume fraction [6]. The thickness of Jute/PLA composite with $\mathrm{V}_{\mathrm{f}}$ of $37.39 \%$ was $1.47 \mathrm{~mm}$ manufactured at $185^{\circ} \mathrm{C}$, but composite manufactured at $235{ }^{\circ} \mathrm{C}$ with $\mathrm{V}_{\mathrm{f}}$ of $53.65 \%$ was only $1.03 \mathrm{~mm}$ thick also reported better elastic modulus. This affirms that resin viscosity improves with processing temperature and ensures better impregnation even with larger fiber content.

The above findings conclude that the strength of a composite material largely depends on the fabrication conditions and affects the load carrying capacity of the fiber. However, there is lack of a similar study on different processing temperature and pressure on mechanical properties of Pure PLA coupons out of compression molding. An independent study of pure PLA and how processing temperature and pressure in compression molding influences its characteristics in comparison to a PLA part that is manufactured by 3D printing is lacking and this gap is the focus of this research project. Table 2 below summarizes several studies investigating compression molding process parameters and their impact on mechanical performance of the final parts. 
Table 2: A summary of the literature survey investigating the impact of process parameters on mechanical performance of the final parts.

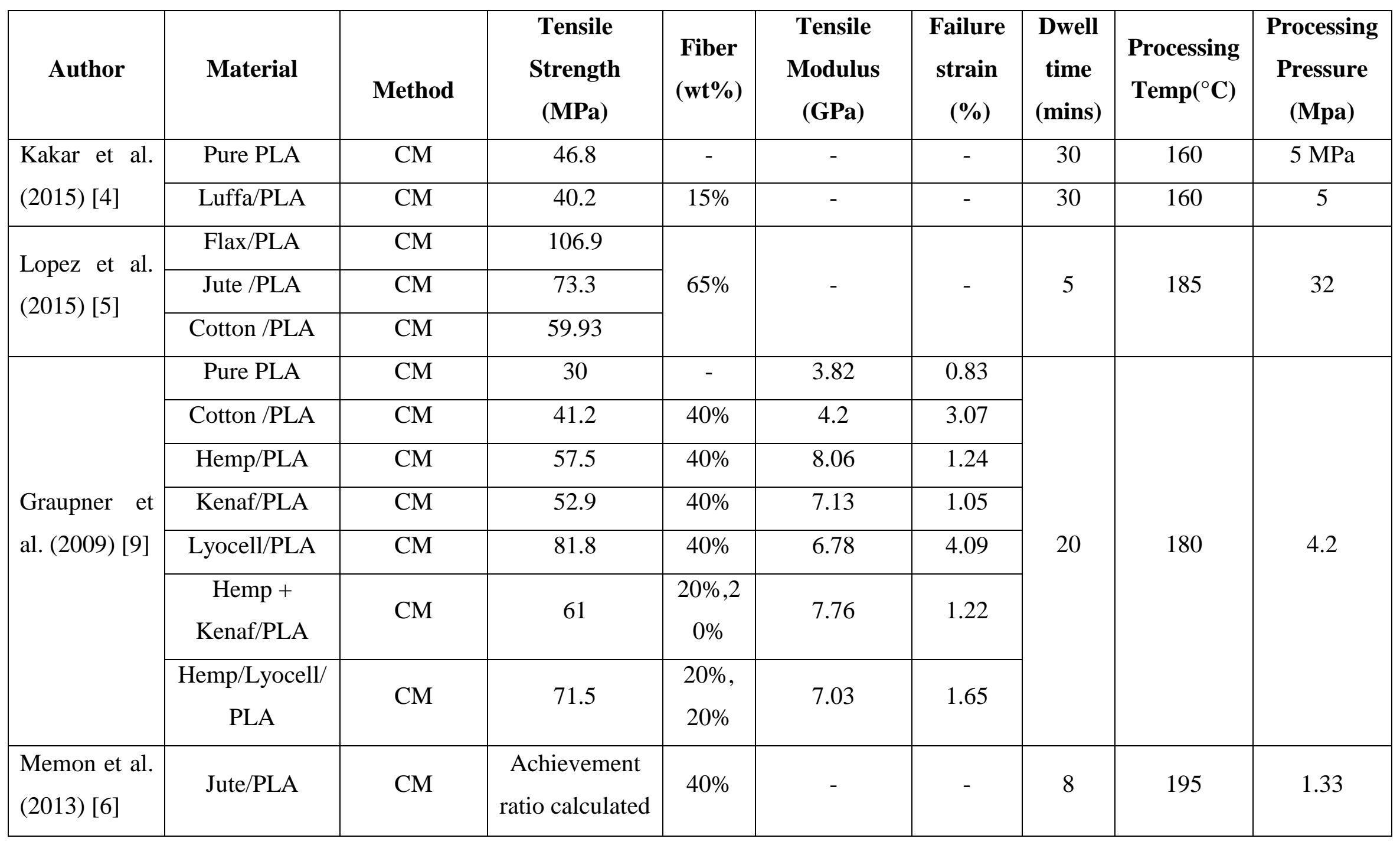




\begin{tabular}{|c|c|c|c|c|c|c|c|c|c|}
\hline Author & Material & Method & $\begin{array}{c}\text { Tensile } \\
\text { Strength } \\
\text { (MPa) }\end{array}$ & $\begin{array}{l}\text { Fiber } \\
(w t \%)\end{array}$ & $\begin{array}{c}\text { Tensile } \\
\text { Modulus } \\
\text { (GPa) }\end{array}$ & $\begin{array}{c}\text { Failure } \\
\text { strain } \\
(\%)\end{array}$ & $\begin{array}{l}\text { Dwell } \\
\text { time } \\
(\text { mins })\end{array}$ & $\begin{array}{l}\text { Processing } \\
\operatorname{Temp}\left({ }^{\circ} \mathrm{C}\right)\end{array}$ & $\begin{array}{c}\text { Processing } \\
\text { Pressure } \\
\text { (Mpa) }\end{array}$ \\
\hline $\begin{array}{l}\text { Ochin (2008) } \\
{[15]}\end{array}$ & Kenaf/PLA & Hot Press & 223 & $70 \%$ & - & - & 10 & 160 & 10 \\
\hline \multirow{2}{*}{$\begin{array}{l}\text { Srebrenkoska } \\
\text {.et al. } \\
\text { (2009) [3] }\end{array}$} & Kenaf/PLA & \multirow[b]{2}{*}{$\mathrm{CM}$} & $28.3 \pm 4.8$ & $30 \%$ & $2.87 \pm 0.2$ & - & 10 & 175 & 5 \\
\hline & Rice hull/PLA & & $26.7 \pm 2.0$ & $30 \%$ & $2.76 \pm 0.1$ & - & & & \\
\hline \multirow{2}{*}{$\begin{array}{l}\text { Graupner et } \\
\text { al. (2016) } \\
{[10]}\end{array}$} & Lyocell/PLA & $\mathrm{CM}$ & $92-118$ & 40 & - & - & 5 & $180 / 25$ & 17.5 \\
\hline & Lyocell/PLA & IM & $45-66$ & 40 & - & - & - & 180 & 48 \\
\hline $\begin{array}{l}\text { Raj et al. } \\
(2018)[14]\end{array}$ & Pure PLA & FDM & 48.6 & & 3.1 & - & - & - & - \\
\hline \multirow{2}{*}{$\begin{array}{c}\text { L.G Blok } \\
\text { (2018) } \\
{[7]}\end{array}$} & $\begin{array}{c}\text { Short Carbon/ } \\
\text { PLA }\end{array}$ & FDM & $\begin{array}{c}33.5 \\
( \pm 2.5 \%)\end{array}$ & 6 & $\begin{array}{c}1.85 \\
( \pm 2.5 \%)\end{array}$ & \multirow{2}{*}{-} & \multirow{2}{*}{-} & \multirow{2}{*}{ - } & \multirow{2}{*}{ - } \\
\hline & $\begin{array}{c}\text { Cont. Carbon/ } \\
\text { PLA }\end{array}$ & FDM & $\begin{array}{c}986 \\
( \pm 8.3 \%)\end{array}$ & 27 & $\begin{array}{c}62.5 \\
( \pm 4.9 \%)\end{array}$ & & & & \\
\hline
\end{tabular}




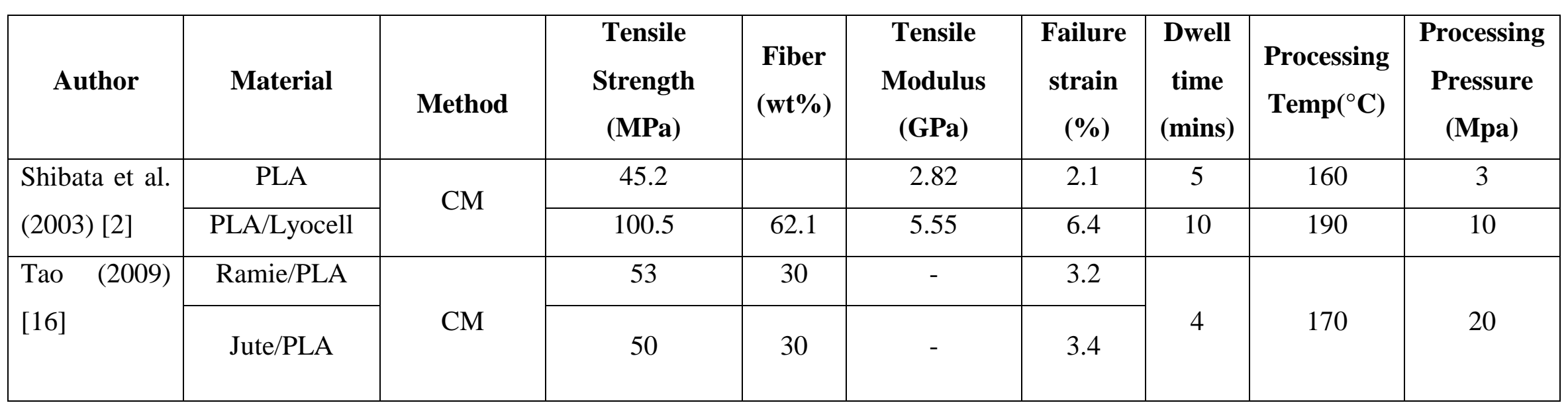




\section{MATERIALS AND METHODOLOGY}

This section describes the material, equipment for manufacturing PLA specimens and testing of PLA specimens as per ASTM D4703-16 [18] in order to determine its tensile strength properties for different operating levels of temperature, pressure and dwell time were considered as the control factors.

\subsection{MATERIAL}

PLA polymer pellets, grade Ingeo 4043D, used for this study are supplied by NatureWorks LLc with a peak melting temperature between 145 to $160^{\circ} \mathrm{C}$, and glass transition temperature of $55-60{ }^{\circ} \mathrm{C}$. The properties of PLA are listed in Table 3 below:

\section{Table 3: Properties of PLA pellets.}

\begin{tabular}{|l|c|}
\hline \multicolumn{2}{|c|}{ Physical Properties (Ingeo Resin) } \\
\hline Specific Gravity, g/cc & 1.24 \\
\hline MFR, g/10 min & 6 \\
\hline Relative Viscosity & 4.0 \\
\hline Clarity & Transparent \\
\hline Peak Melt Temperature, ${ }^{\circ} \mathrm{C}$ & $145-160$ \\
\hline Glass Transition Temperature, ${ }^{\circ} \mathrm{C}$ & $55-60$ \\
\hline
\end{tabular}

\subsection{MOLD MATERIAL}

The mold used for compression molding is a 12 in. $\times 12$ in. plate with 0.5 in. thickness. Considering ease of machining and ability to retain strength at high temperatures, the mold was machined on a low carbon steel plate. 
As per ASTM D638-14, Standard test method for Tensile properties of Plastic, Type-I specimen are considered for testing. A total of eleven specimen cavities for standard dog-bone shaped test specimens which are modeled using SolidWorks. Each specimen cavity has a depth of 0.14in., which is within the specified thickness limit of $3.2 \pm 0.4 \mathrm{~mm}$ as shown in Figure 3 .

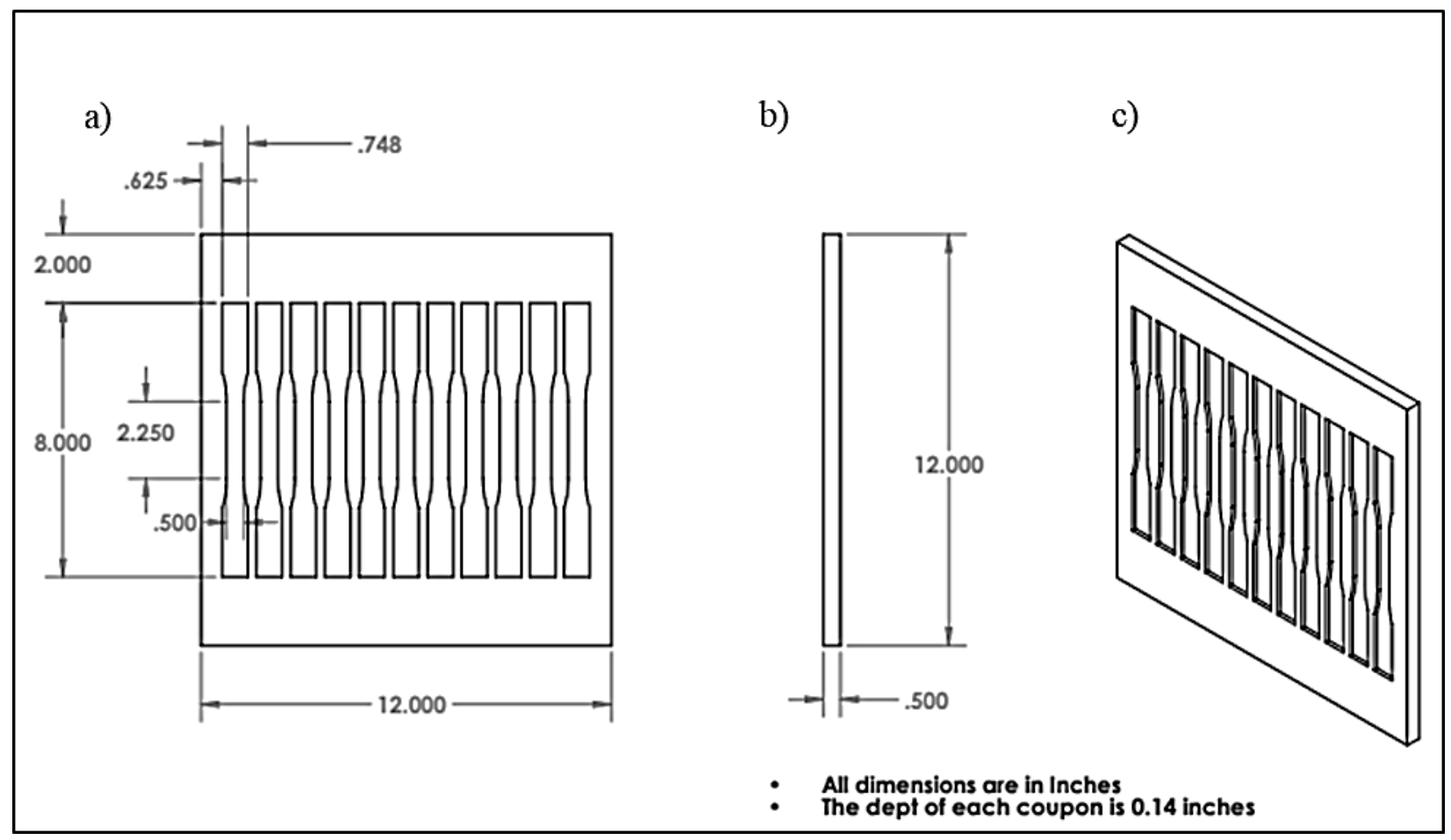

Figure 3: Drawing of the $12 \times 12$ in. mold with eleven coupon cavities: (a) Front view; (b) Side view; (c) isometric.

\subsection{COMPRESSION MOLDING MACHINE}

Compression molding machine used is Carver auto series, with two heated platens of $15 \times 15 \mathrm{in}$. dimensions and can provide a maximum clamping force of 30 ton.lbf. It also has a built-in control panel which allows modifying each cycle of fabrication by changing the temperature and pressure as required. 


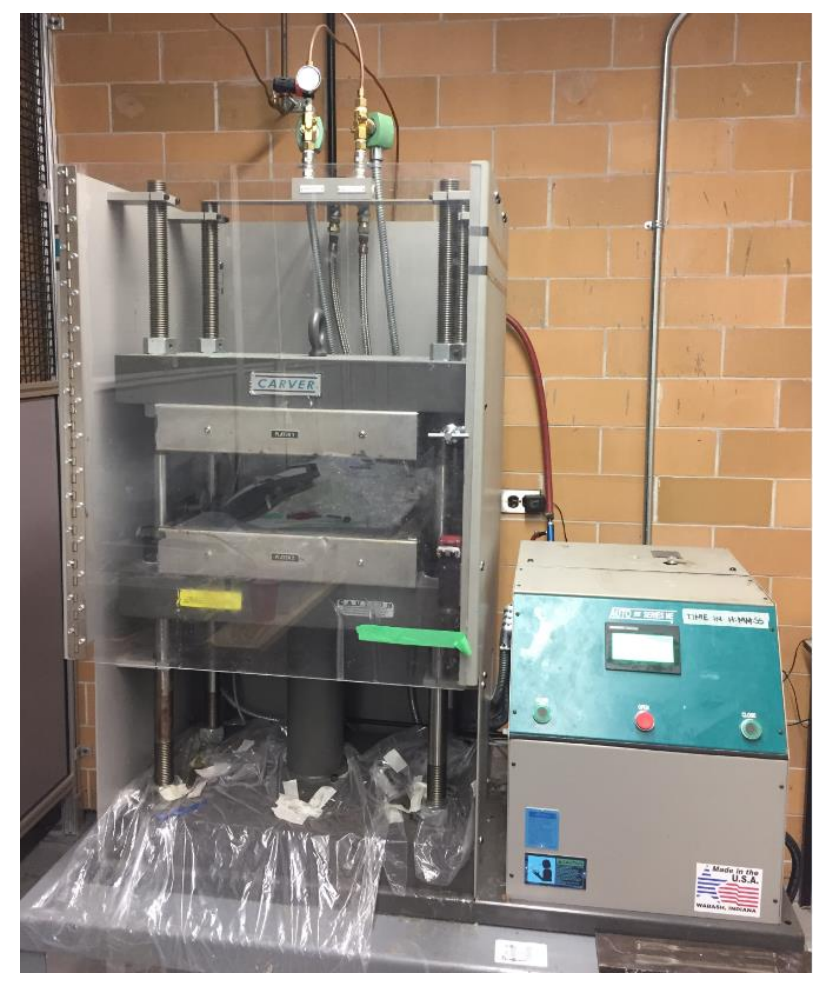

Figure 4: Carver auto series machine.

As discussed in literature survey section, several manufacturing parameters that influence the mechanical properties and quality of the finished material in compression molding methodology are:

- Processing temperature

- Processing pressure

- Dwell time

- Cooling time.

Based on the literature survey, it is understood that there is a need to optimize a quick and efficient processing methodology for thermoplastics. With this as one of the prime objectives, various molding temperatures, molding pressure with different dwell times were investigated. The cooling temperature for all specimens have been performed with the automated cooling cycle assisted by the compression molding machine and over same period of 10 mins. Table 4 summarizes the manufacturing parameters of the compression molding process used in this study. 


\section{Table 4: Manufacturing Parameters of Compression molding in this study.}

\begin{tabular}{|c|c|c|c|c|}
\hline \multirow{2}{*}{ Levels } & \multicolumn{4}{|c|}{ Factors } \\
\cline { 2 - 5 } & Temp $\left({ }^{\circ} \mathbf{C}\right)$ & $\begin{array}{c}\text { Pressure } \\
(\mathbf{M P a})\end{array}$ & $\begin{array}{c}\text { Dwell Time } \\
(\mathbf{m i n s})\end{array}$ & $\begin{array}{c}\text { Cooling Time } \\
\text { (mins) }\end{array}$ \\
\hline 1 & 150 & 2 & 10 & 10 \\
\hline 2 & 160 & 2.5 & 20 & 10 \\
\hline 3 & 170 & 3 & 30 & 10 \\
\hline
\end{tabular}

To effectively study the effect of each processing parameter, Taguchi's Design of Experiments (DOE) was utilized to optimize the number of required experiments.

The minimum number of experiment to be conducted based on Taguchi's design [14]:

$$
N_{\text {Taguchi }}=1+\sum_{i=1}^{N V}\left(L_{i}-1\right)
$$

Where, $\mathrm{NV}$ is the number of variables,

$L_{i}$ is the number of levels identified for each variable.

$L_{i}$ is three in this case since we have three levels for each factor. Using the above equation, the calculation suggests at least seven experiments are to be performed, but due to the balancing property of Taguchi's orthogonal array, number of experiments must be a multiple of three, thus a total of nine experiment trials are carried out as shown in Table 5. 
Table 5: Test plan for different Processing temperatures, Pressure and Dwell times.

\begin{tabular}{|c|c|c|c|c|}
\hline Expt. No. & $\begin{array}{c}\text { Temp } \\
(\mathbf{C})\end{array}$ & $\begin{array}{c}\text { Pressure } \\
\text { (Mpa) }\end{array}$ & $\begin{array}{c}\text { Dwell Time } \\
\text { (mins) }\end{array}$ & $\begin{array}{c}\text { Cooling } \\
\text { Time } \\
(\mathbf{m i n s})\end{array}$ \\
\hline $\mathbf{1}$ & 150 & 2 & 10 & 10 \\
\hline $\mathbf{2}$ & 150 & 2.5 & 20 & 10 \\
\hline $\mathbf{3}$ & 150 & 3 & 30 & 10 \\
\hline $\mathbf{4}$ & 160 & 2 & 20 & 10 \\
\hline $\mathbf{5}$ & 160 & 2.5 & 30 & 10 \\
\hline $\mathbf{6}$ & 160 & 3 & 10 & 10 \\
\hline $\mathbf{7}$ & 170 & 2 & 30 & 10 \\
\hline $\mathbf{8}$ & 170 & 2.5 & 10 & 10 \\
\hline $\mathbf{9}$ & 170 & 3 & 20 & 10 \\
\hline
\end{tabular}

\subsection{COMPRESSION MOLDING PROCEDURE}

A detailed step-by-step procedure followed in the process to manufacture the PLA specimens is described below:

\section{Step 1:}

The coupon cavity in the mold is compactly filled with PLA pellets, weight equivalent of $20 \mathrm{~g}$ in each cavity, such that on melting there is adequate material to fill the cavity. The mold is then placed between the two hot platens of the Compression molding machine, Carver Auto Series press with 30 tons of clamping force capacity. The mold, with PLA pellets, is heated from room temperature to the processing temperature as shown in the test plan Table 5. 


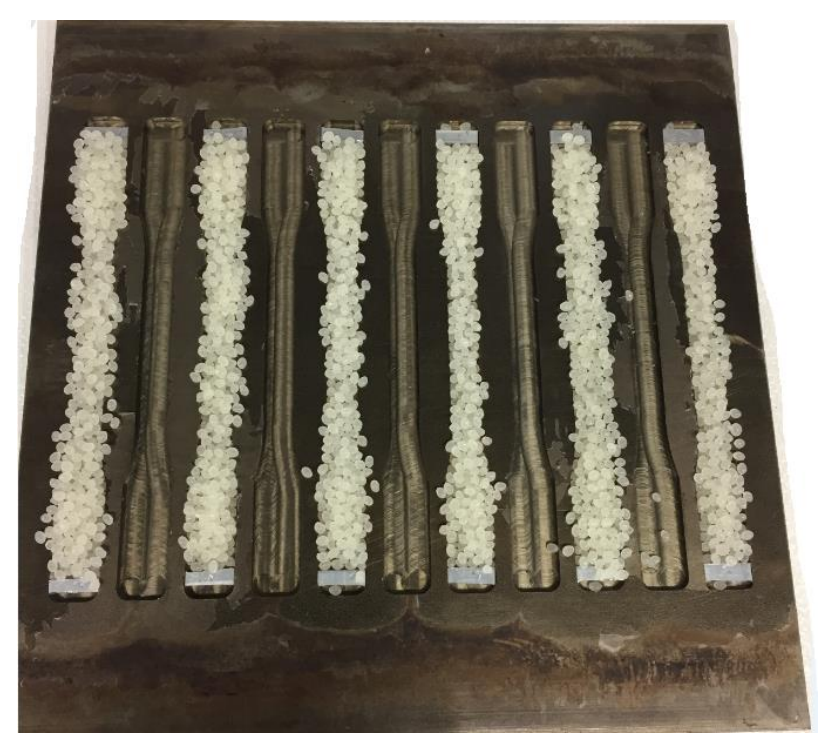

Figure 5: PLA pellets in mold cavity.

\section{Step 2:}

During Pre-heating, when the two platens of the compression molding get to the set required temperature, a thermocouple is used to ensure that the mold has also reached the desired temperature per trials. Once the desired temperature is attained, it is held for 10 minutes to allow even heating of the PLA.

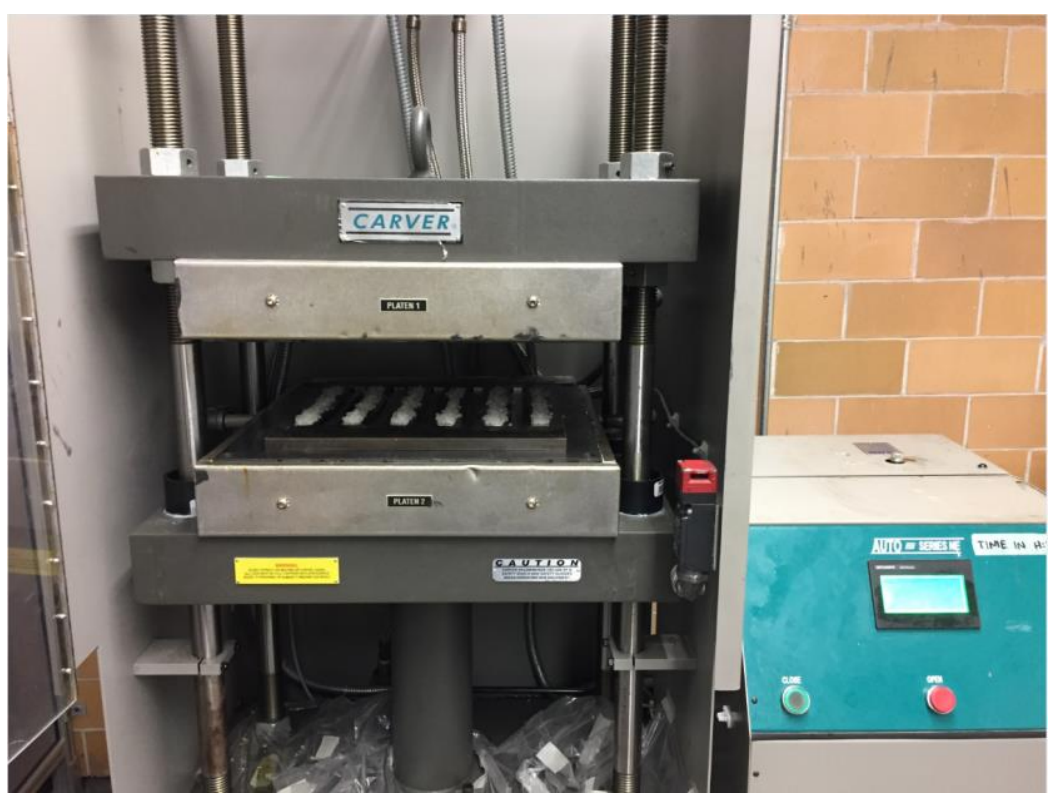

Figure 6: Picture showing the mold containing the PLA pellets in the machine. 


\section{Step 3:}

The top and bottom platens, with mold filled with PLA centrally aligned and covered with an aluminum plate, is closed to apply the desired force on the mold to generate the required pressure. The contact area between the platen and the mold is: 11.5 in. $\times 12$ in. $=138 \mathrm{in}^{2}$ As a result, the required load to reach processing pressure per Table 5 can be calculated as below

Table 6: Pressure applied in the study for compression molding.

\begin{tabular}{|c|c|c|}
\hline \multirow{2}{*}{$\begin{array}{c}\text { Load } \\
\text { (lbf) }\end{array}$} & \multicolumn{2}{|c|}{ Pressure } \\
\cline { 2 - 3 } & psi & MPa \\
\hline 40000 & 289.86 & 2.00 \\
\hline 50000 & 362.32 & 2.50 \\
\hline 60000 & 434.78 & 3.00 \\
\hline
\end{tabular}

\section{Step 4:}

The last segment of the manufacturing cycle is the cooling cycle where the PLA specimens are cooled from the processing temperature to room temperature $\left(30^{\circ} \mathrm{C}\right)$ in 10 minutes, at a rate 12 $14{ }^{\circ} \mathrm{C} / \mathrm{min}$ depending on the process temperature $\left(150-170{ }^{\circ} \mathrm{C}\right)$.

\section{Step 5:}

Demolding the PLA samples after they are cooled to room temperature in the machine. For easy removal of specimens, small metal tabs added on the either side of the cavity, this ensures no damage to the specimen due to demolding.

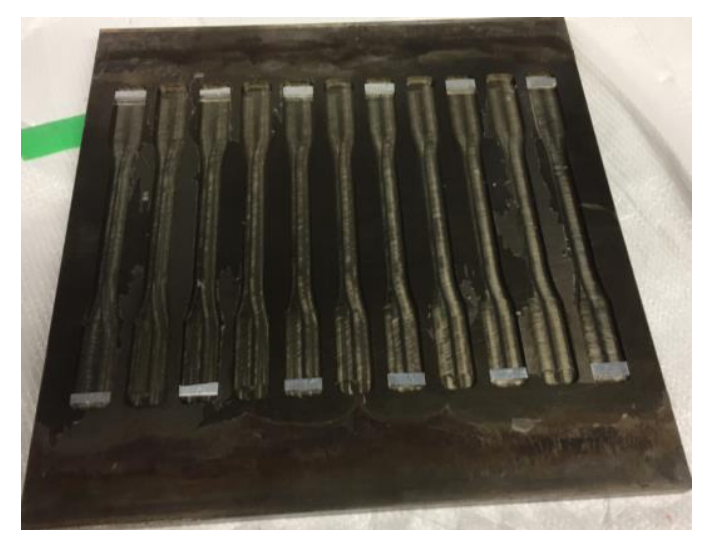

Figure 7: The mold with the metal tabs. 
Fabrication of the sample as per the test plan in Table 5 is carried out and details of process cycle (temperature and pressure cycles) of each batch is discussed below:

\section{Batch-1:}

Manufacturing cycle of Batch-1 is shown in Figure 8. The platens of the compression molding machine are heated to the processing temperature of $150{ }^{\circ} \mathrm{C}$. This temperature is held constant for 10 mins to ensure uniform heating of PLA pellets. A thermocouple is used to ensure that the mold has attained the processing temperature. Then the platens are closed and a pressure of $2.0 \mathrm{MPa}$ is applied. A steady curing stage at $150{ }^{\circ} \mathrm{C}$ and $2 \mathrm{MPa}$ pressure, is performed for $10 \mathrm{mins}$ followed by cooling stage from $150{ }^{\circ} \mathrm{C}$ to room temperature in $10 \mathrm{mins}$ under constant pressure of $2 \mathrm{MPa}$. The finished specimens are demolded from the mold.
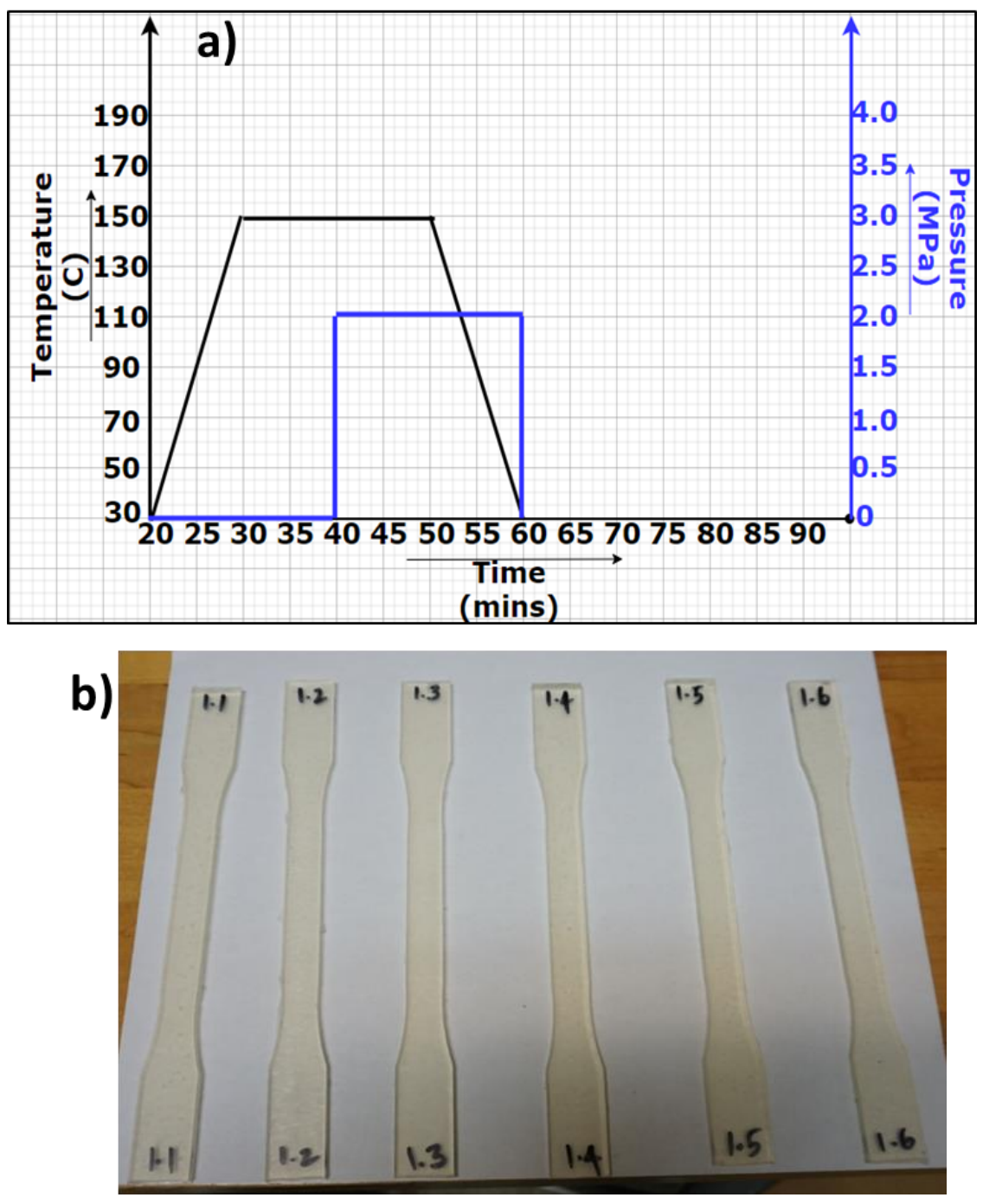

Figure 8: Batch-1: a) temperature and pressure cycle; b) Fabricated Batch-1 specimens after demolding. 


\section{Batch-2:}

Manufacturing cycle of Batch-2 is shown in Figure 9. The platens of the compression molding machine are heated to the processing temperature of $150{ }^{\circ} \mathrm{C}$. This temperature is held constant for 10 mins to ensure uniform heating of PLA pellets. A thermocouple is used to ensure that the mold has attained the processing temperature. Then the platens are closed and a pressure of $2.5 \mathrm{MPa}$ is applied. A steady curing stage at $150{ }^{\circ} \mathrm{C}$ and $2.5 \mathrm{MPa}$ pressure, is performed for $20 \mathrm{mins}$, followed by cooling stage from $150^{\circ} \mathrm{C}$ to room temperature in 10 mins under constant pressure of $2.5 \mathrm{MPa}$. The finished specimens are demolded from the mold.
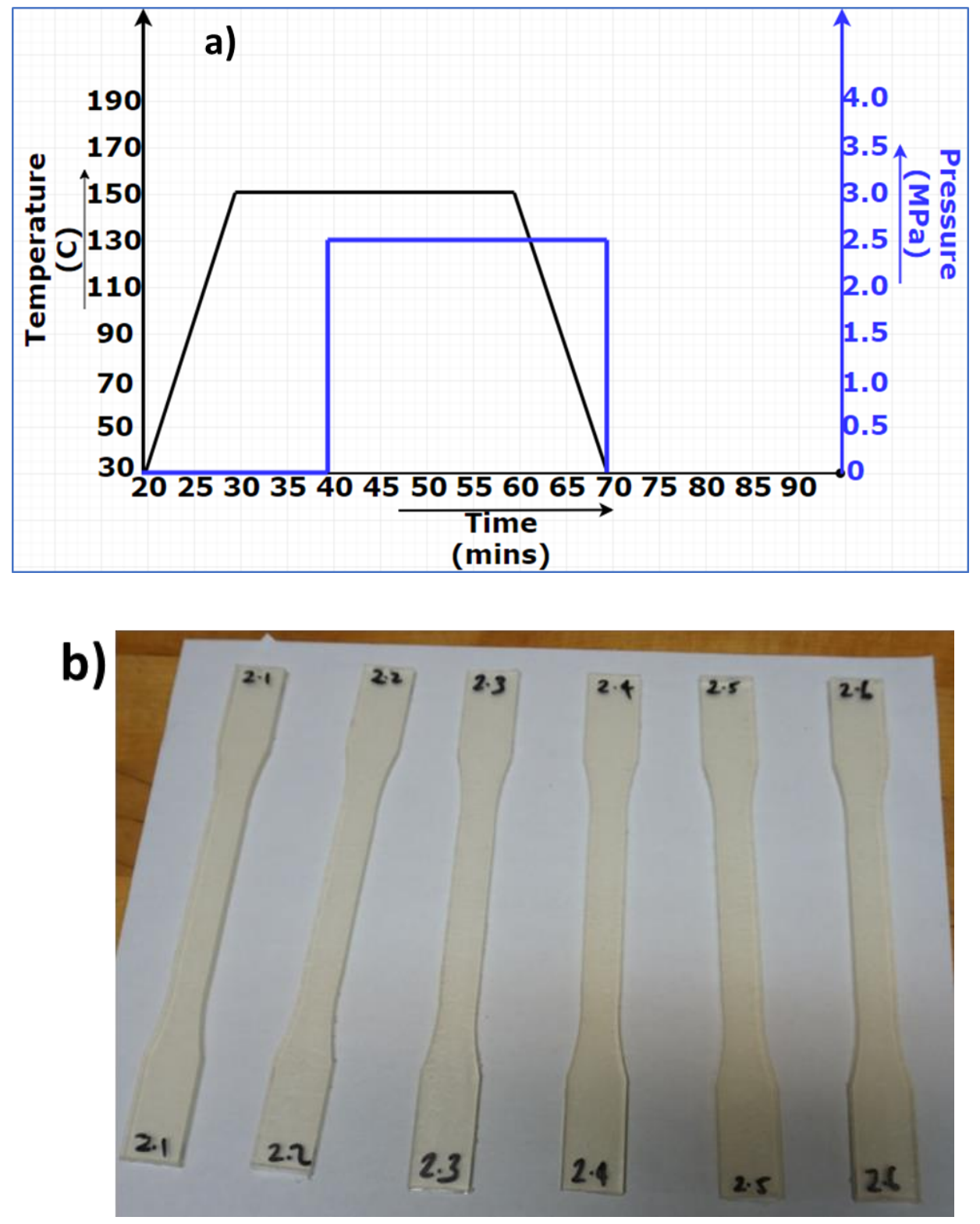

Figure 9: Batch-2: a) temperature and pressure cycle; b) Fabricated Batch-2 specimens after demolding. 


\section{Batch-3:}

Manufacturing cycle of Batch-3 is shown in Figure 10. The platens of the compression molding machine are heated to the processing temperature of $150{ }^{\circ} \mathrm{C}$. This temperature is held constant for 10 mins to ensure uniform heating of PLA pellets. A thermocouple is used to ensure that the mold has attained the processing temperature. Then the platens are closed and a pressure of $3 \mathrm{MPa}$ is applied. A steady curing stage at $150{ }^{\circ} \mathrm{C}$ and $3 \mathrm{MPa}$ pressure, is performed for $30 \mathrm{mins}$, followed by cooling stage from $150{ }^{\circ} \mathrm{C}$ to room temperature in 10 mins under constant pressure of $3 \mathrm{MPa}$. The finished specimens are demolded from the mold.
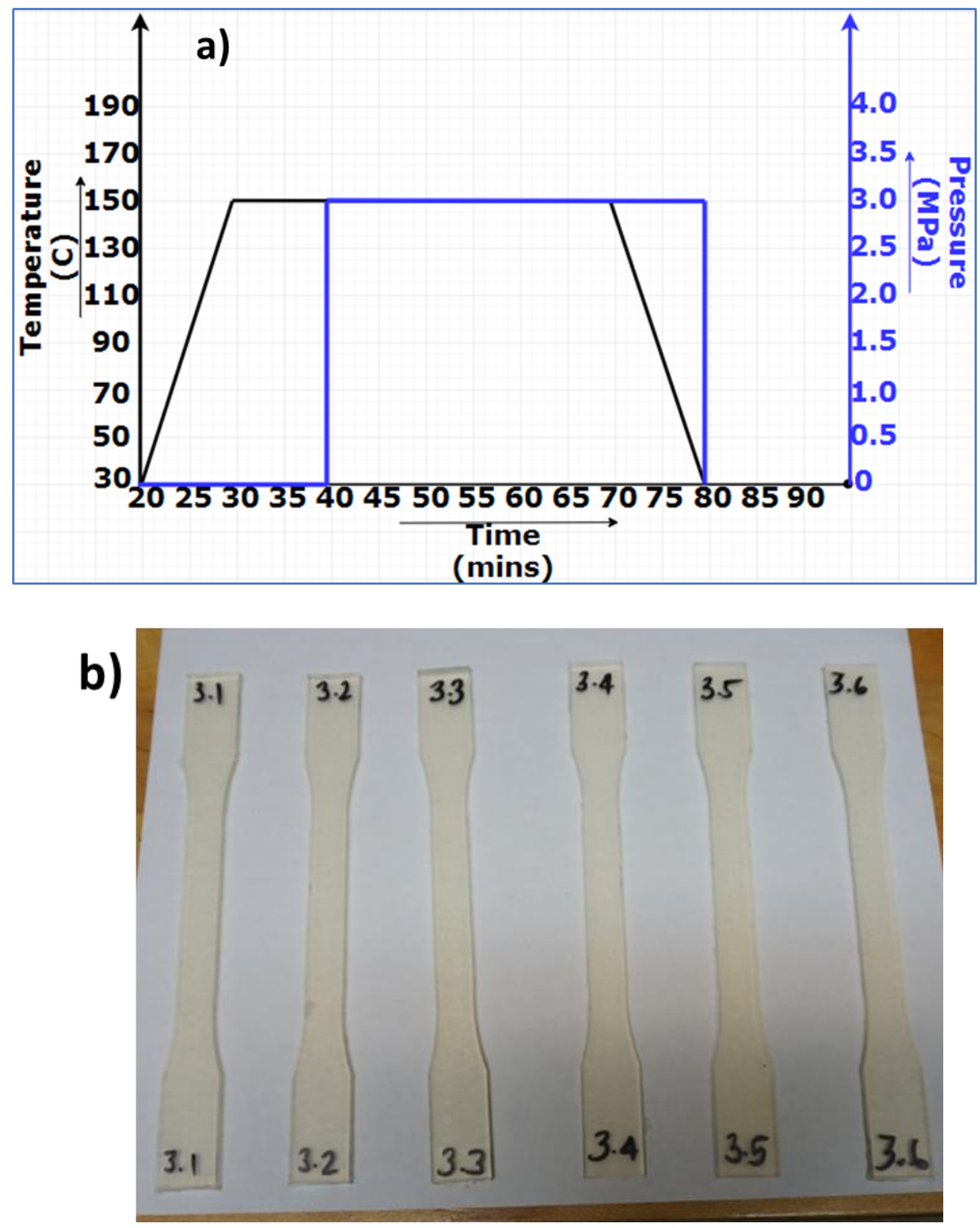

Figure 10: Batch-3: a) temperature and pressure cycle; b) Fabricated Batch-3 specimens after demolding. 


\section{Batch-4:}

Manufacturing cycle of Batch-4 is shown in Figure 11. The platens of the compression molding machine are heated to the processing temperature of $160{ }^{\circ} \mathrm{C}$. This temperature is held constant for 10 mins to ensure uniform heating of PLA pellets. A thermocouple is used to ensure that the mold has attained the processing temperature. Then the platens are closed and a pressure of $2 \mathrm{MPa}$ is applied. A steady curing stage at $160{ }^{\circ} \mathrm{C}$ and $2 \mathrm{MPa}$ pressure, is performed for $20 \mathrm{mins}$, followed by cooling stage from $150{ }^{\circ} \mathrm{C}$ to room temperature in 10 mins under constant pressure of $2 \mathrm{MPa}$. The finished specimens are demolded at room temperature from the mold.
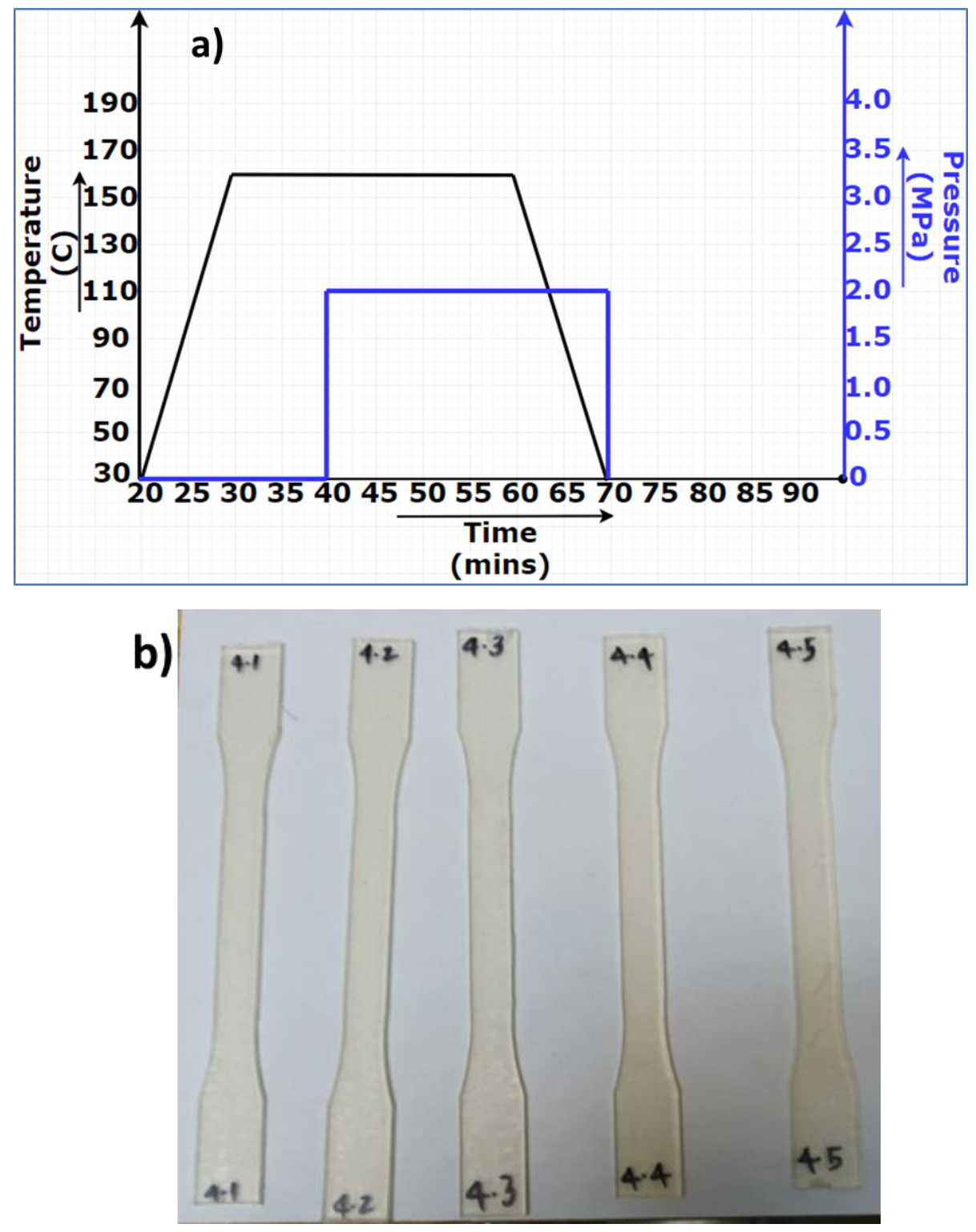

Figure 11: Batch-4: a) temperature cycle and pressure cycle; b) Fabricated batch- 4 specimens after demolding. 


\section{Batch-5:}

Manufacturing cycle of Batch-5 is shown in Figure 12. The platens of the compression molding machine are heated to the processing temperature of $160{ }^{\circ} \mathrm{C}$. This temperature is held constant for 10 mins to ensure uniform heating of PLA pellets. A thermocouple is used to ensure that the mold has attained the processing temperature. Then the platens are closed and a pressure of $2.5 \mathrm{MPa}$ is applied. A steady curing stage at $160{ }^{\circ} \mathrm{C}$ and $2.5 \mathrm{MPa}$ pressure, is performed for $30 \mathrm{mins}$, followed by cooling stage from $160^{\circ} \mathrm{C}$ to room temperature in 10 mins under constant pressure of $2.5 \mathrm{MPa}$. The finished specimens are demolded from the mold.
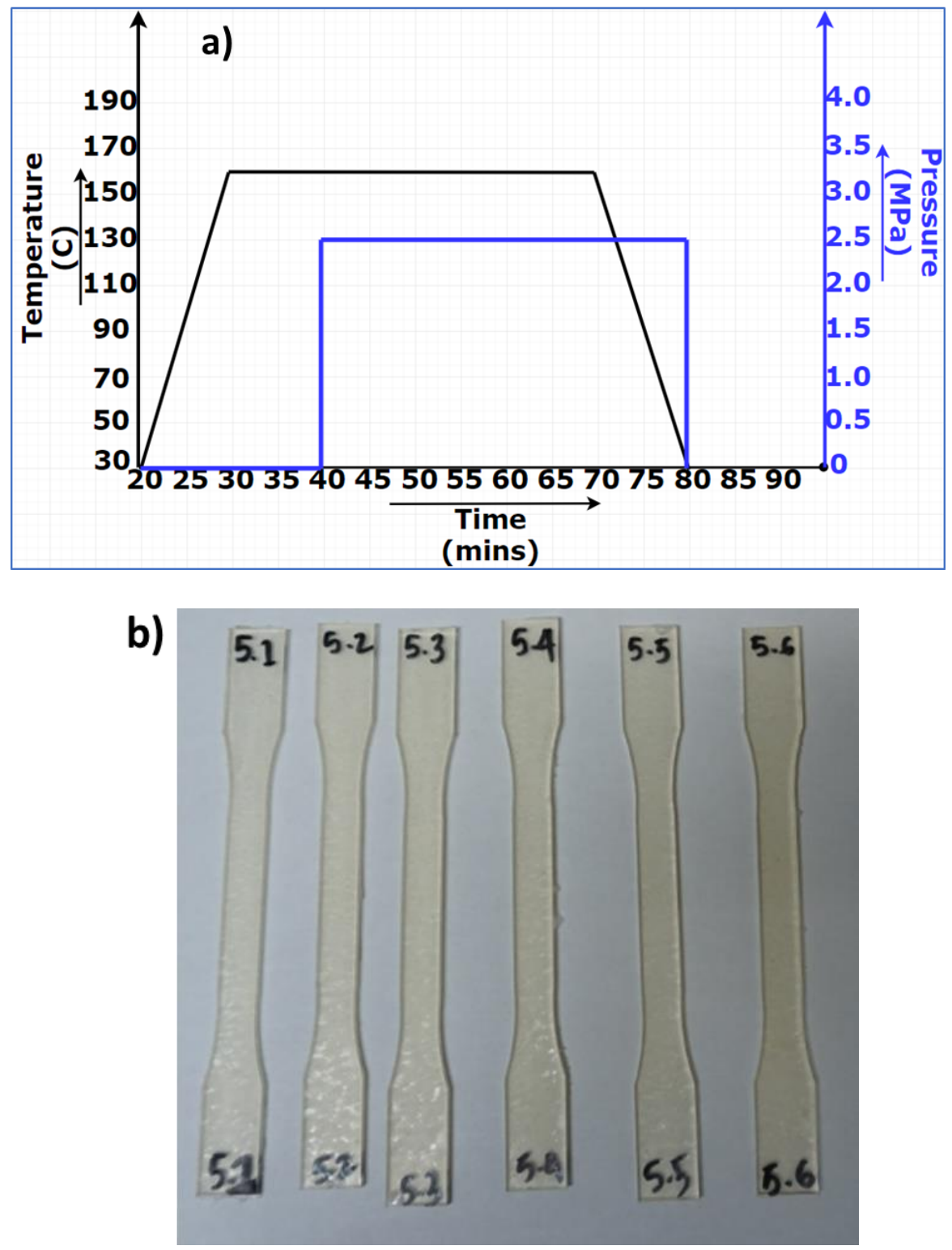

Figure 12: Batch-5: a) temperature cycle, and pressure cycle; b) Fabricated batch-5 specimens after demolding. 


\section{Batch-6:}

Manufacturing cycle of Batch-6 is shown in Figure 13. The platens of the compression molding machine are heated to the processing temperature of $160{ }^{\circ} \mathrm{C}$. This temperature is held constant for 10 mins to ensure uniform heating of PLA pellets. A thermocouple is used to ensure that the mold has attained the processing temperature. Then the platens are closed and a pressure of $3 \mathrm{MPa}$ is applied. A steady curing stage at $150{ }^{\circ} \mathrm{C}$ and $3 \mathrm{MPa}$ pressure, is performed for $10 \mathrm{mins}$, followed by cooling stage from $160{ }^{\circ} \mathrm{C}$ to room temperature in 10 mins under constant pressure of $3 \mathrm{MPa}$. The finished specimens are demolded from the mold.
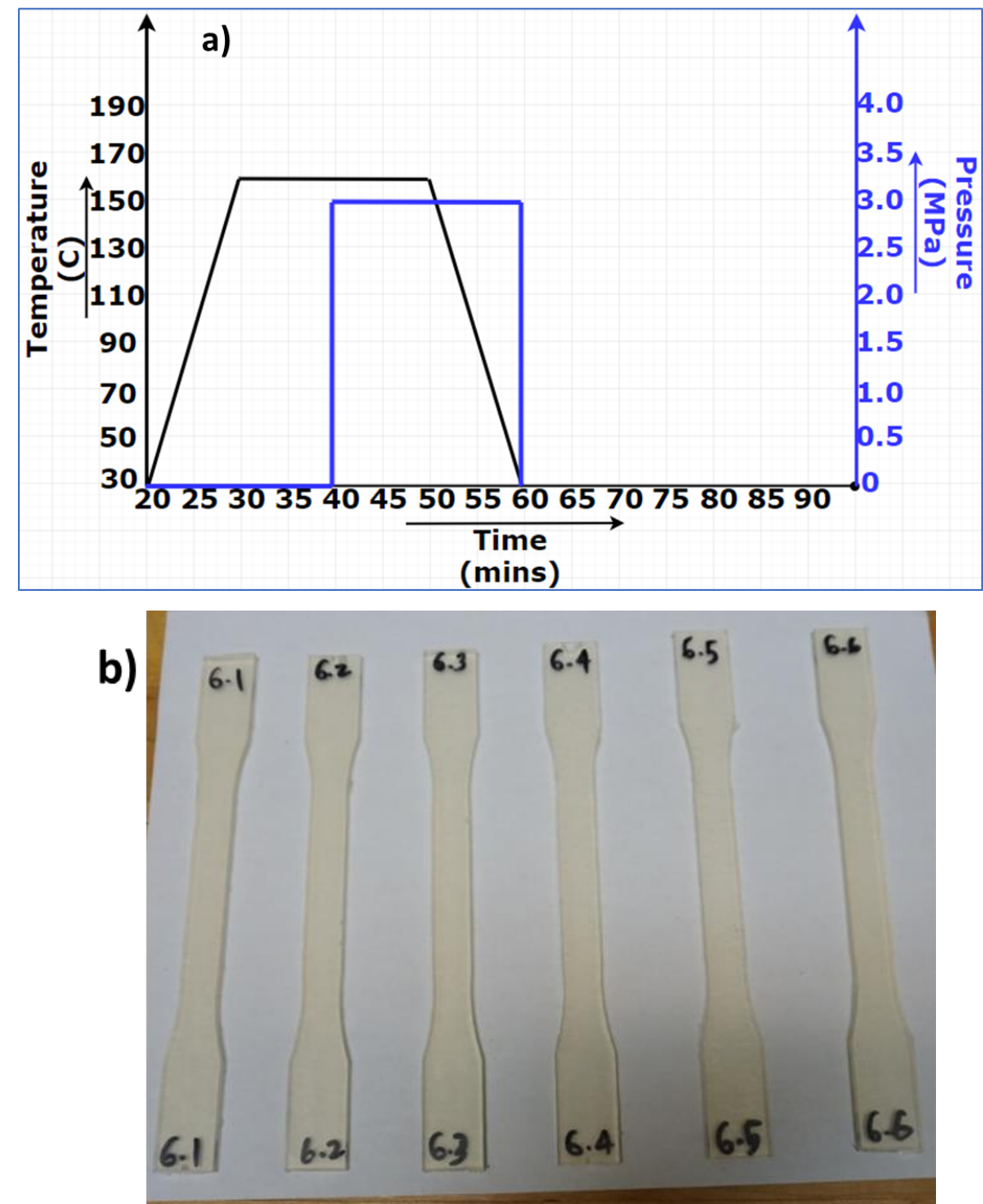

Figure 13: Batch-6: a) temperature cycle, and pressure cycle; b) Fabricated batch-6 specimens after demolding. 


\section{Batch-7:}

Manufacturing cycle of Batch-7 is shown in Figure 14. The platens of the compression molding machine are heated to the processing temperature of $170{ }^{\circ} \mathrm{C}$. This temperature is held constant for 10 mins to ensure uniform heating of PLA pellets. A thermocouple is used to ensure that the mold has attained the processing temperature. Then the platens are closed and a pressure of $2 \mathrm{MPa}$ is applied. A steady curing stage at $170{ }^{\circ} \mathrm{C}$ and $2 \mathrm{MPa}$ pressure, is performed for $30 \mathrm{mins}$. Followed by cooling stage from $170{ }^{\circ} \mathrm{C}$ to room temperature in 10 mins under constant pressure of $2 \mathrm{MPa}$. The finished specimens are demolded from the mold.
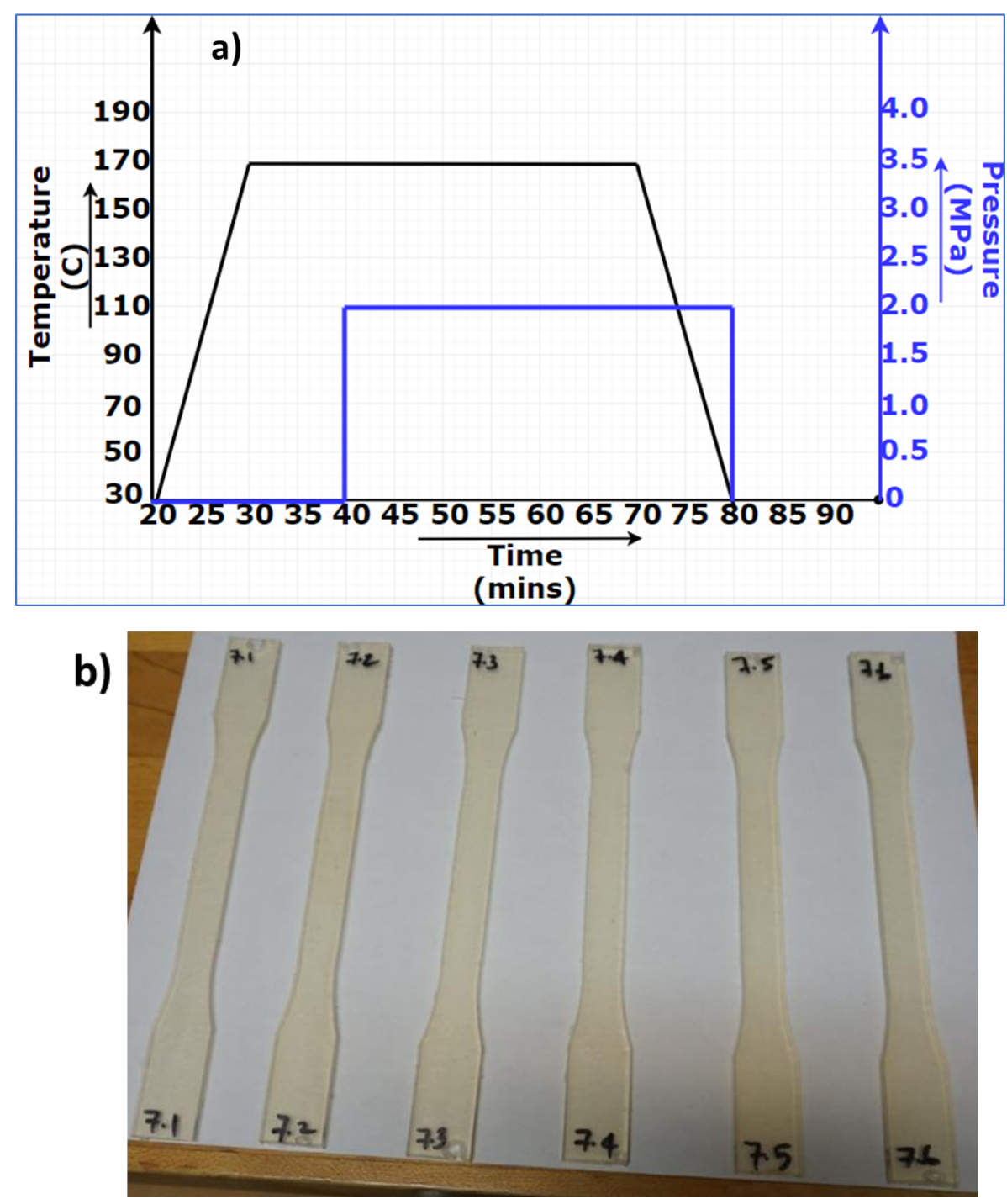

Figure 14: Batch-7: a) temperature cycle, and pressure cycle; b) Fabricated batch-7 specimens after demolding. 


\section{Batch-8:}

Manufacturing cycle of Batch-8 is shown in Figure 15. The platens of the compression molding machine are heated to the processing temperature of $170{ }^{\circ} \mathrm{C}$. This temperature is held constant for 10 mins to ensure uniform heating of PLA pellets. A thermocouple is used to ensure that the mold has attained the processing temperature. Then the platens are closed and a pressure of $2.5 \mathrm{MPa}$ is applied. A steady curing stage at $170{ }^{\circ} \mathrm{C}$ and $2.5 \mathrm{MPa}$ pressure, is performed for $10 \mathrm{mins}$, followed by cooling stage from $170{ }^{\circ} \mathrm{C}$ to room temperature in 10 mins under constant pressure of $2.5 \mathrm{MPa}$. The finished specimens are demolded from the mold.
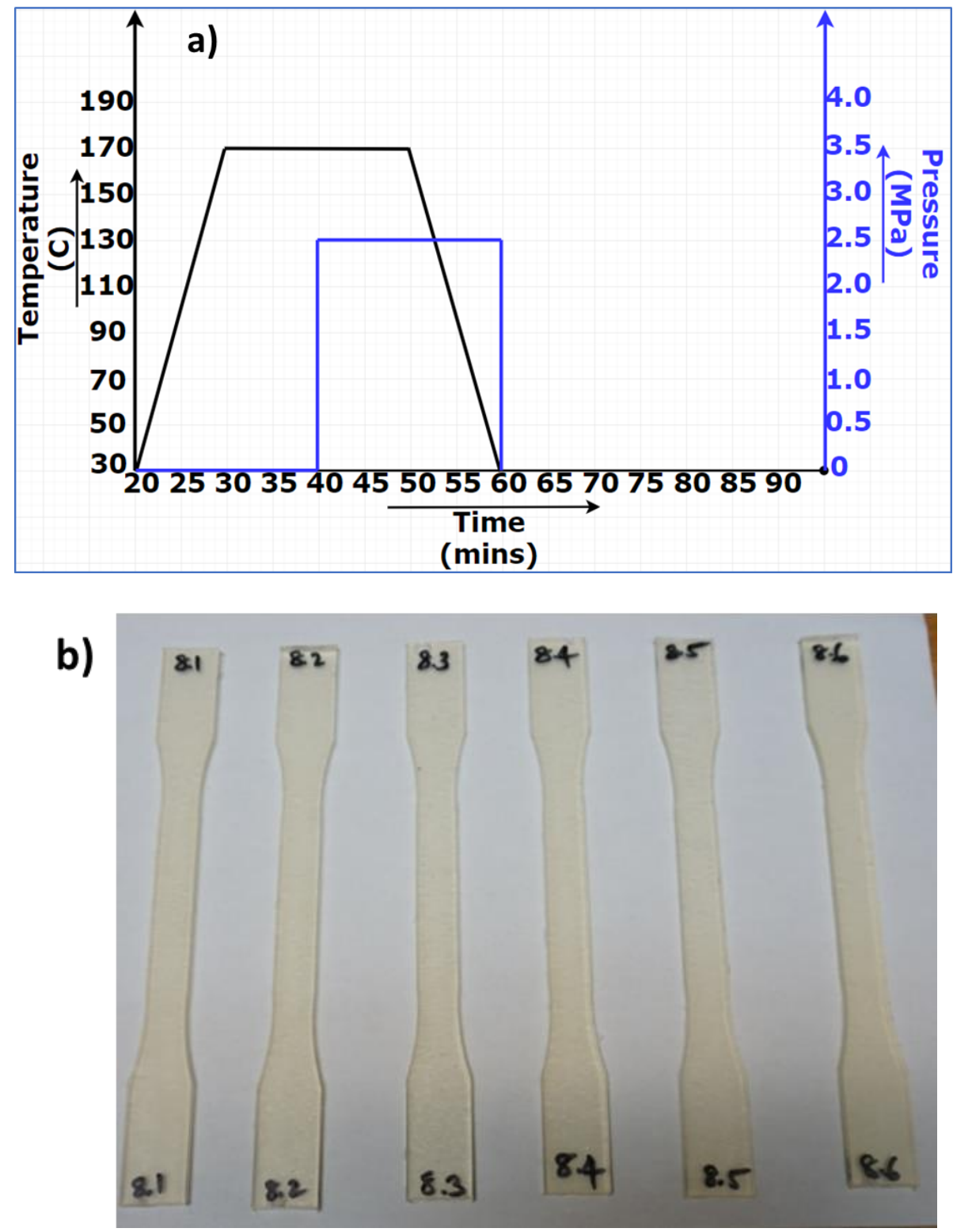

Figure 15: Batch-8: a) temperature cycle, and pressure cycle; b) Fabricated batch-8 specimens after demolding. 


\section{Batch-9:}

Manufacturing cycle of Batch-9 is shown in Figure 16. The platens of the compression molding machine are heated to the processing temperature of $150{ }^{\circ} \mathrm{C}$. This temperature is held constant for 10 mins to ensure uniform heating of PLA pellets. A thermocouple is used to ensure that the mold has attained the processing temperature. Then the platens are closed and a pressure of $2.5 \mathrm{MPa}$ is applied. A steady curing stage at $150{ }^{\circ} \mathrm{C}$ and $2.5 \mathrm{MPa}$ pressure, is performed for 20 mins. Followed by cooling stage from $150{ }^{\circ} \mathrm{C}$ to room temperature in 10 mins while under constant pressure of 2.5 MPa. The finished specimens are demolded from the mold.
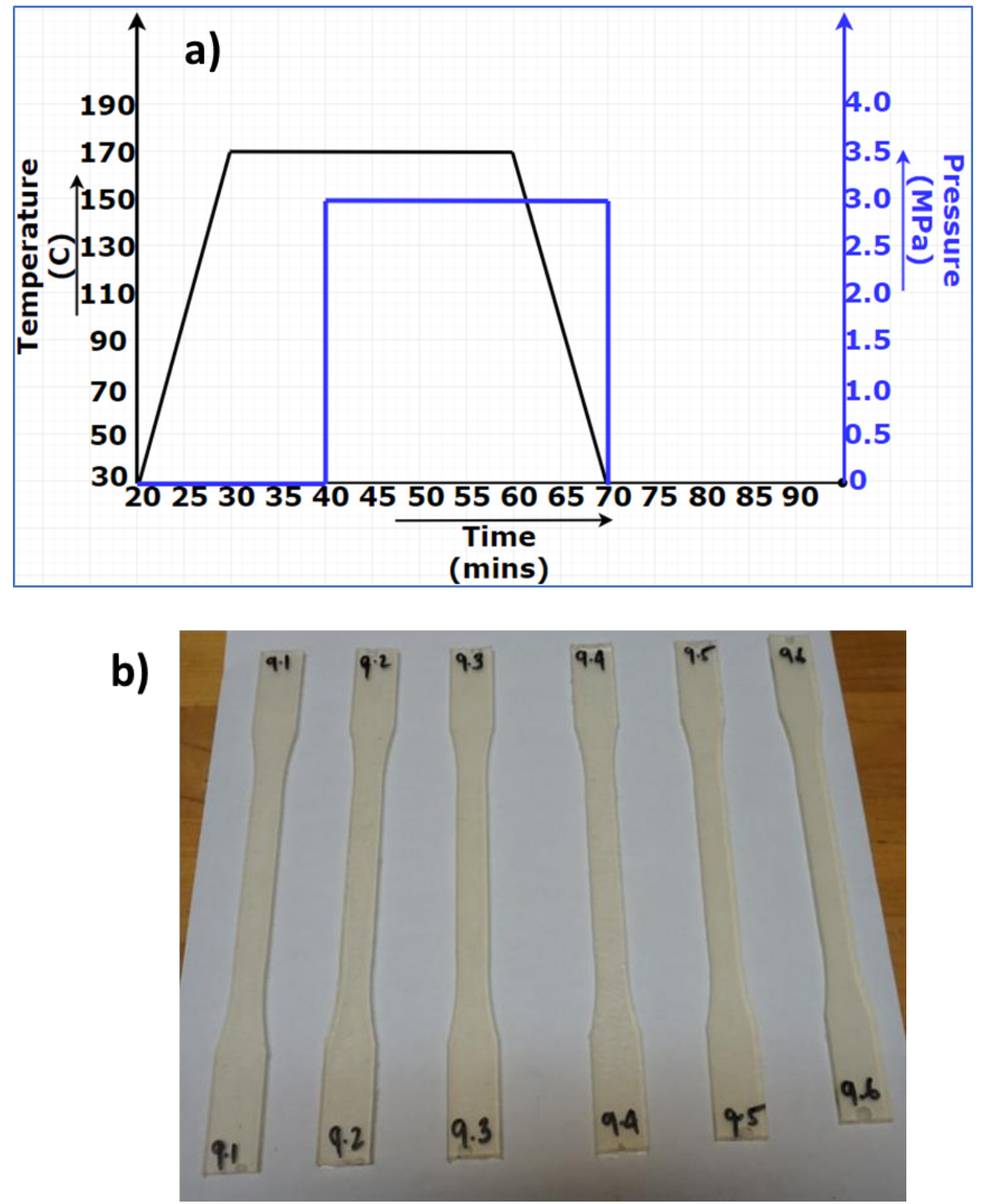

Figure 16: Batch-9: a) temperature cycle, and pressure cycle; b) Fabricated batch-9 specimens after demolding. 


\subsection{D PRINTED SPECIMENS}

Six pure PLA specimens manufactured by fused filament fabricated (3D printed) as per ASTM D638-14 specifications are tested for tensile strength properties. 3D specimens were manufactured using a Prusa i3 Mk2S printer. The 3D model of the specimen is passed to Simplify3D to prepare G-codes for FFF 3D printing. Figure 17 visualizes materials deposition for specimens in Simplify3D software. Raster angle of $0^{\circ}$ for all specimens.

Figure 17: Materials deposition visualization in Simplify3D for $0^{\circ}$ layer-specimen.

The material deposition orientations can be seen in the manufactured specimens (along the specimen length) that correspond to the simulated ones in Simplify3D. The 3D printed specimen used in this study is as shown in Figure 18.

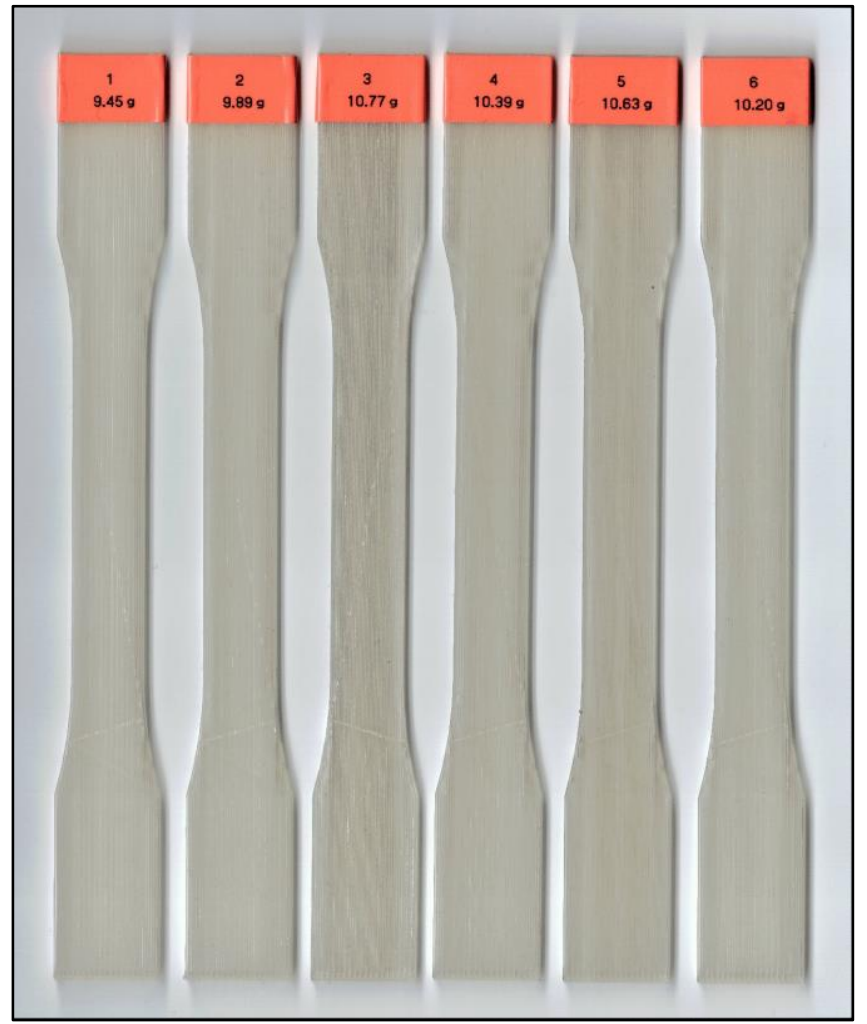

Figure 18: Six 3D printed PLA specimens. 
Optimized 3D printing process parameters were found in previous studies [18] and were used here to manufacture specimens per ASTM D638.Design and manufacturing process parameters are summarized in Table 7.

Table 7: Manufacturing and design parameters for specimen 3D printing.

\begin{tabular}{|l|c|l|c|}
\hline $\begin{array}{c}\text { Manufacturing/design } \\
\text { Parameter }\end{array}$ & Value & $\begin{array}{c}\text { Manufacturing/design } \\
\text { Parameter }\end{array}$ & Value \\
\hline Print direction & XYZ & Material & PLA \\
\hline Filament diameter & $1.75 \mathrm{~mm}$ & Nozzle diameter & $0.4 \mathrm{~mm}$ \\
\hline Layer height & $0.14 \mathrm{~mm}$ & Nozzle temperature & $215^{\circ} \mathrm{C}$ \\
\hline Bed temperature & 60 & Cooling & No fan cooling \\
\hline Printing speed & $\begin{array}{c}2400 \\
\mathrm{~mm} / \mathrm{min}\end{array}$ & Infill \% & $100 \%$ \\
\hline Raster angle & $0^{\circ}$ & \multicolumn{2}{|c|}{} \\
\hline
\end{tabular}




\section{TESTING}

Compression molded, and 3D printed PLA specimens are tested in a Universal testing machine as per ASTM D638-14 to determine tensile strength, tensile modulus, and failure strain of PLA specimens. A total of 54 compression molded specimens were manufactured as per the test plan in Table 5 for various levels of temperature pressure and dwell time. A united UTM machine as shown in Figure 19 is used for this test with 2000lbs load cell and a constant displacement rate of $5 \mathrm{~mm}(0.2 \mathrm{in}) / \mathrm{min}$ along with an extensometer to measure the elongation of the specimen.

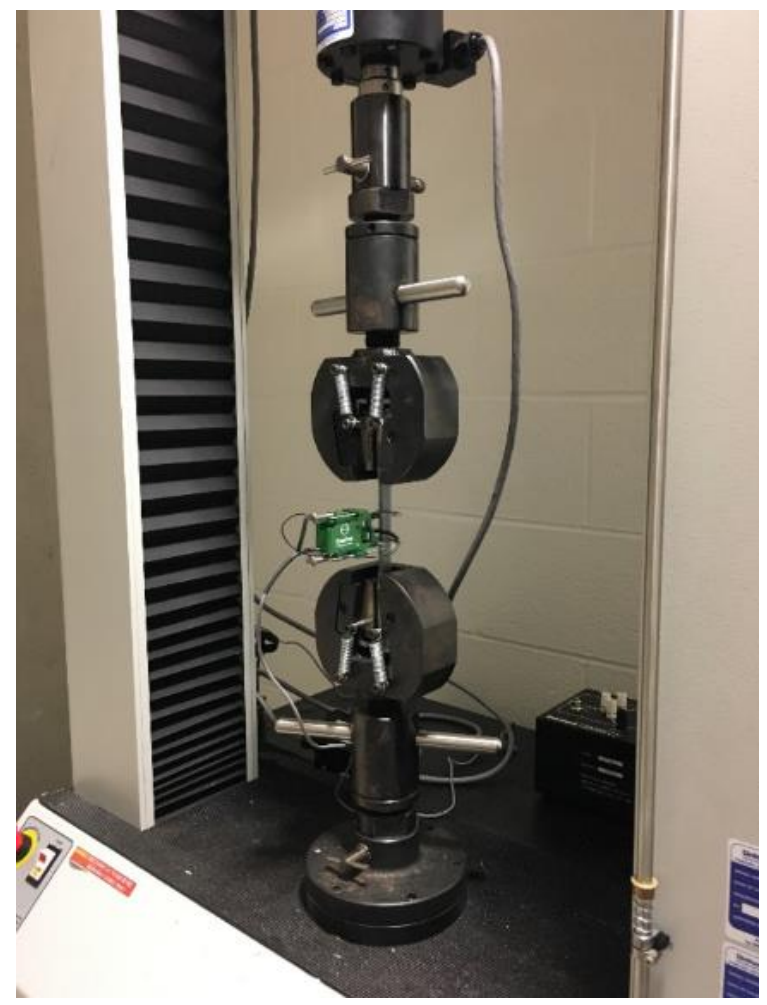

Figure 19: Uniaxial Tensile Testing. 


\section{RESULTS}

This section discusses tensile strength, tensile modulus and failure strain of all 54 compression molded specimens that are manufactured in 9 batches for all varying molding conditions of temperature, pressure and dwell time. A comparison of tensile testing results with 3D printed PLA specimens is performed and a brief discussion of both results is presented.

Results obtained for each batch along with the picture of specimens are discussed below. In order to obtain the value of tensile modulus, data points of the stress vs extension graph were fit to a regression value, $R^{2}$ of 0.999 (three significant figures per ASTM D638-14).

\subsection{BATCH-1}

Tensile strength, tensile modulus and failure strain of all specimens of Batch-1 are listed in Table 8 with the resulting stress-strain graph in Figure 20 and a picture of coupons after failure are shown in Figure 21. Tensile strength of all specimen in this batch are in the same range with highest tensile strength of $59.5 \mathrm{MPa}$ and the lowest is $57.5 \mathrm{MPa}$ for specimen \#1.3 and \#1.1, respectively. The highest tensile modulus of $3.36 \mathrm{GPa}$ was obtained for \#1.5 and the lowest for \#1.6 with 3.01GPa. Specimen \#1.6 with lowest tensile modulus of 3.01 GPa has the highest failure strain of $2.09 \%$.

Table 8: Tensile strength, modulus and failure strain for Batch-1 specimens.

\begin{tabular}{|c|c|c|c|c|c|c|}
\hline Batch no: & Specimen \# & $\begin{array}{c}\text { Maximum } \\
\text { Force } \\
(\mathbf{l b s})\end{array}$ & $\begin{array}{c}\text { Area } \\
\left(\mathbf{i n}^{2}\right)\end{array}$ & $\begin{array}{c}\text { Strength } \\
(\mathbf{M P a})\end{array}$ & $\begin{array}{c}\text { Tensile } \\
\text { Modulus } \\
(\mathbf{G P a})\end{array}$ & $\begin{array}{c}\text { Failure } \\
\text { Strain } \\
(\%)\end{array}$ \\
\hline & 1.1 & 662.7 & 0.08 & 57.5 & 3.21 & 1.93 \\
& 1.2 & 602.7 & 0.07 & 58.4 & 3.19 & 1.98 \\
1 & 1.3 & 653.6 & 0.08 & 59.5 & 3.21 & 2.04 \\
& 1.4 & 664.9 & 0.08 & 57.6 & 3.33 & 1.97 \\
& 1.5 & 660.4 & 0.08 & 58.2 & 3.36 & 1.95 \\
& 1.6 & 572.7 & 0.07 & 59.1 & 3.01 & 2.09 \\
\hline
\end{tabular}




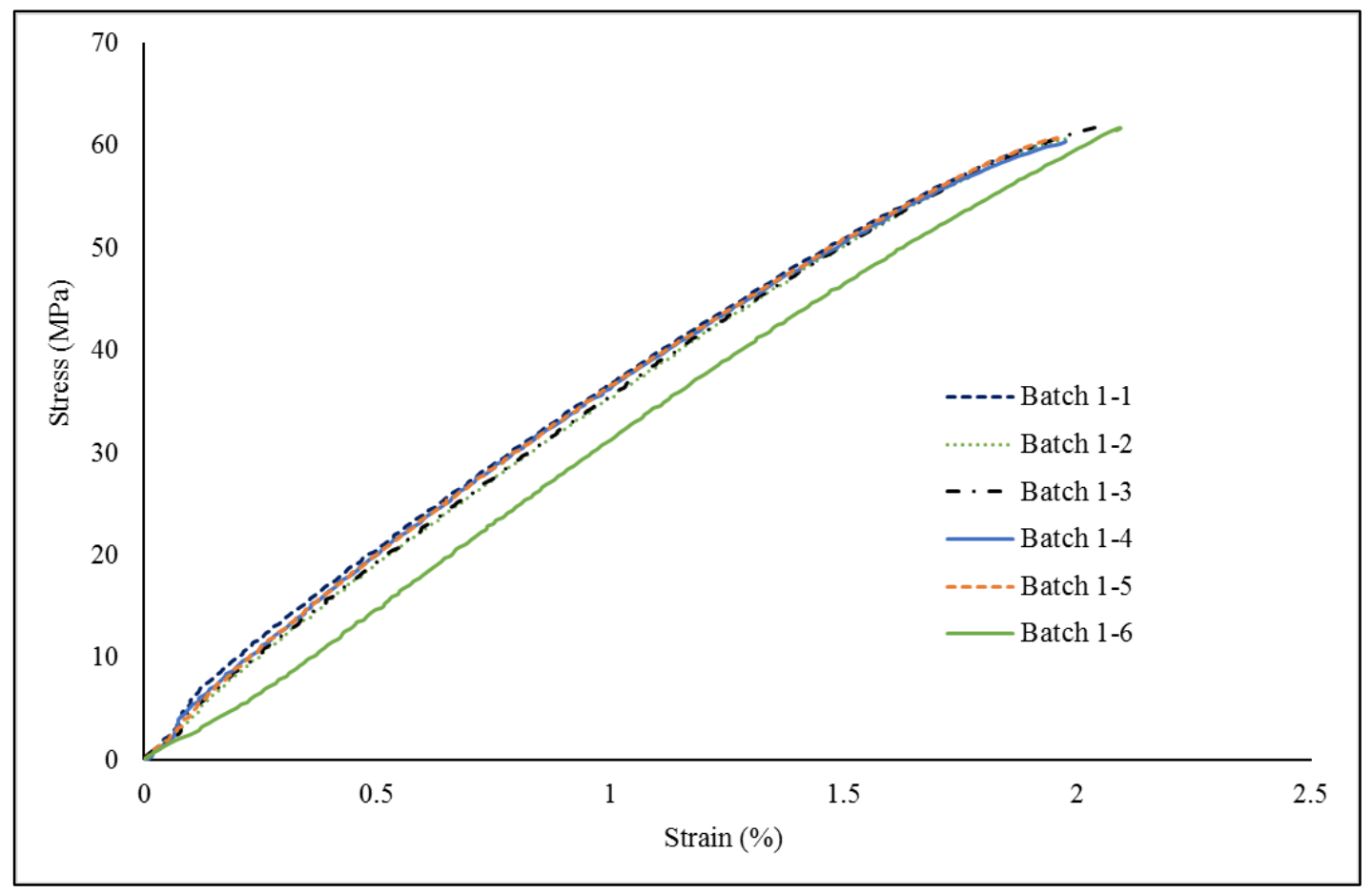

Figure 20: Stress-strain curve of Batch-1 specimens.

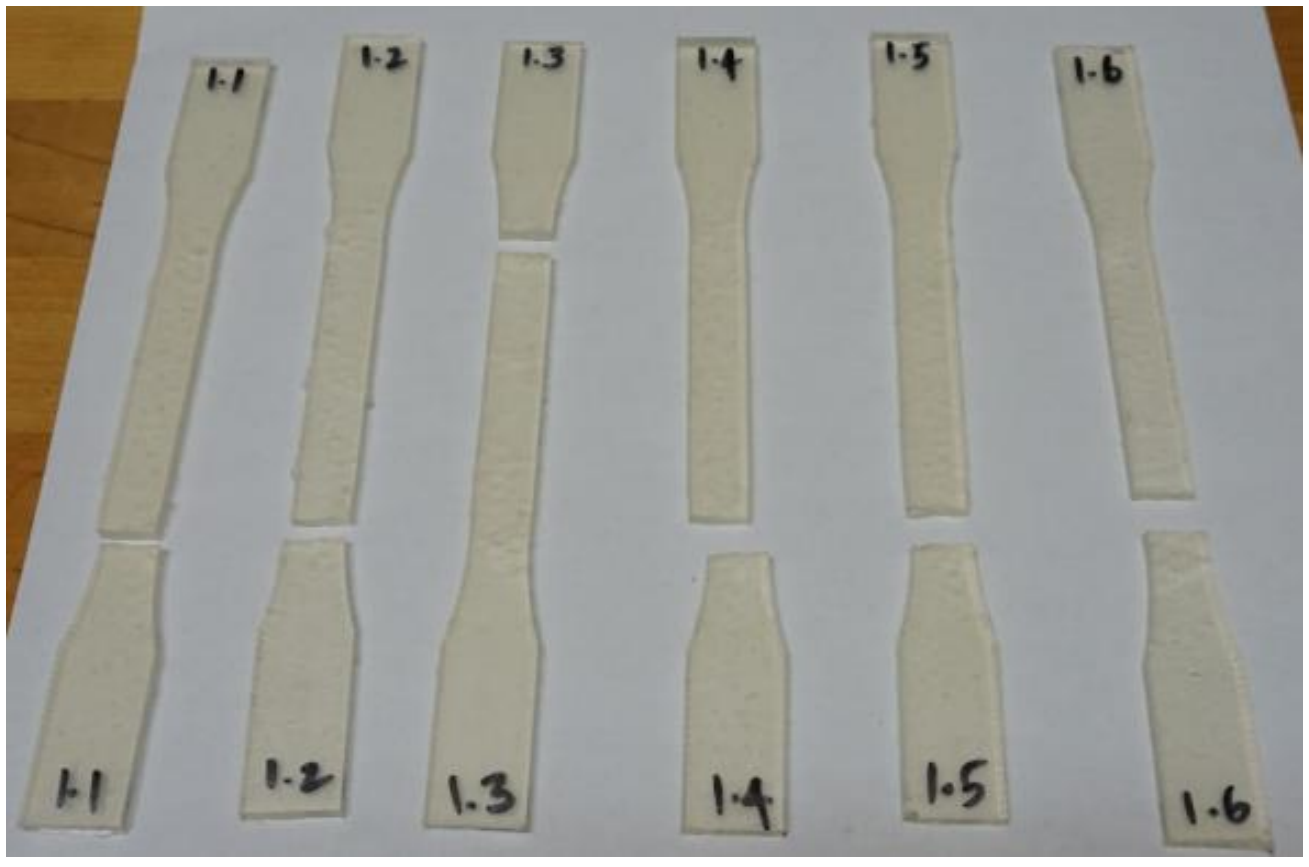

Figure 21: Fractured specimens of Batch-1 after tensile test. 


\section{2 ВАТCH-2}

Tensile strength, tensile modulus and failure strain of all specimens of Batch-2 are listed in Table 9 with the resulting stress-strain graph shown in Figure 22 and a picture of coupons after failure are shown in Figure 23. Tensile strength of all specimen in this batch are in the same range with the highest tensile strength recorded was $60.7 \mathrm{MPa}$ for specimen \#2.4 with an elongation of $2.2 \%$. The lowest tensile strength value in this batch was $56.0 \mathrm{MPa}$ with an elongation of $1.94 \%$ for specimen \#2.2. The highest tensile modulus was obtained for specimen \#2.3 with $3.5 \mathrm{GPa}$ and 59.9 MPa of tensile strength. Specimen \#2.3 recorded the highest failure strain of 2.22\%.

Table 9: Tensile strength, modulus and failure strain for Batch-2 specimens.

\begin{tabular}{|c|c|c|c|c|c|c|}
\hline Batch no: & Specimen \# & $\begin{array}{c}\text { Maximum } \\
\text { Force } \\
(\mathbf{l b s})\end{array}$ & $\begin{array}{c}\text { Area } \\
\left.\mathbf{( i n}^{2}\right)\end{array}$ & $\begin{array}{c}\text { Strength } \\
(\mathbf{M P a})\end{array}$ & $\begin{array}{c}\text { Tensile } \\
\text { Modulus } \\
(\mathbf{G P a})\end{array}$ & $\begin{array}{c}\text { Failure } \\
\text { Strain } \\
(\mathbf{\%})\end{array}$ \\
\hline & 2.1 & 681.4 & 0.08 & 59.4 & 3.10 & 2.18 \\
& 2.2 & 573.7 & 0.07 & 56.0 & 3.13 & 1.94 \\
2 & 2.3 & 576.5 & 0.07 & 59.9 & 3.52 & 2.22 \\
& 2.4 & 660.3 & 0.08 & 60.7 & 3.20 & 2.20 \\
& 2.5 & 683.7 & 0.08 & 60.5 & 3.25 & 2.21 \\
& 2.6 & 694.1 & 0.08 & 60.4 & 3.45 & 2.17 \\
\hline
\end{tabular}

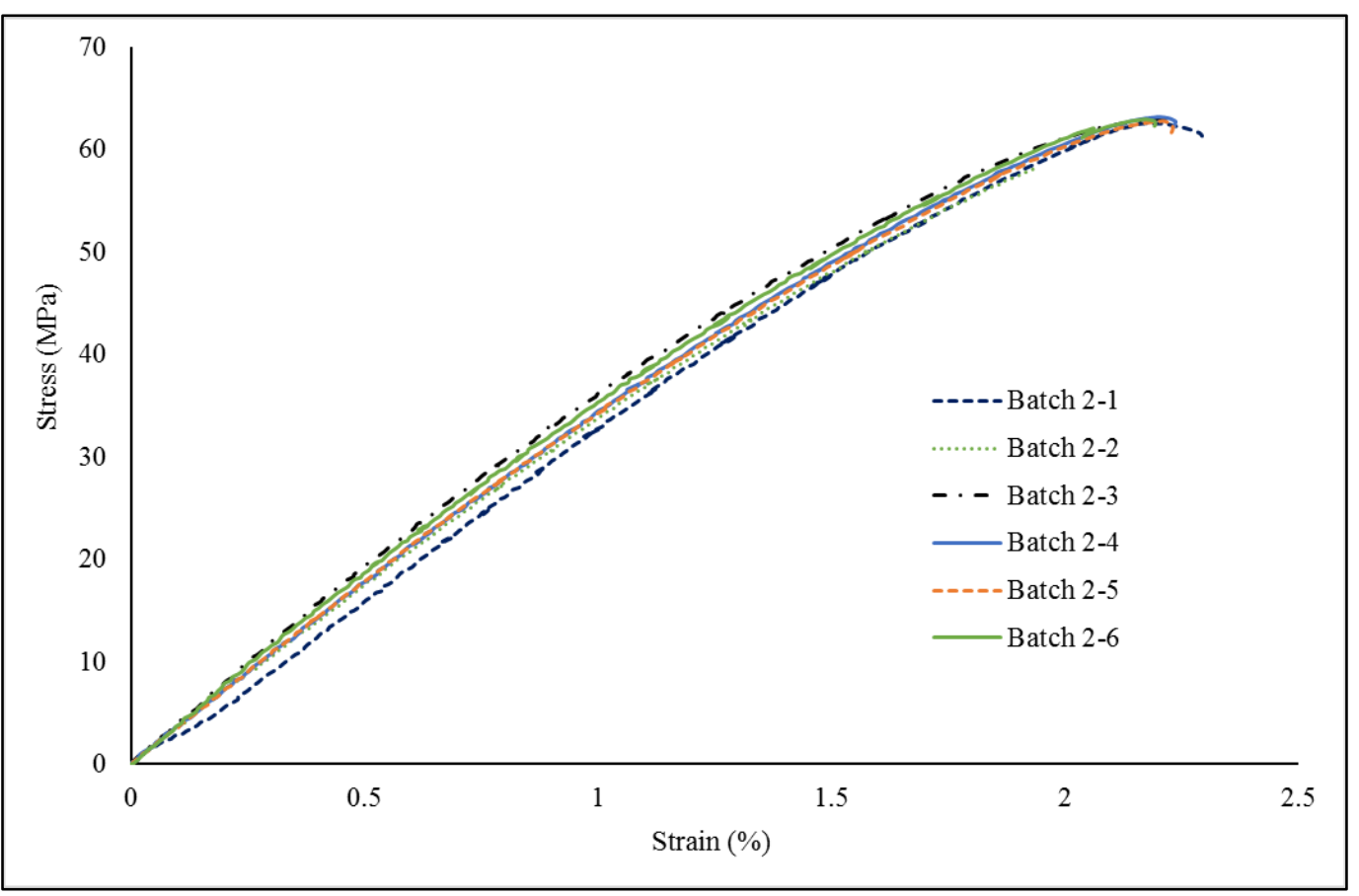

Figure 22: Stress-strain curve of Batch-2 specimens. 


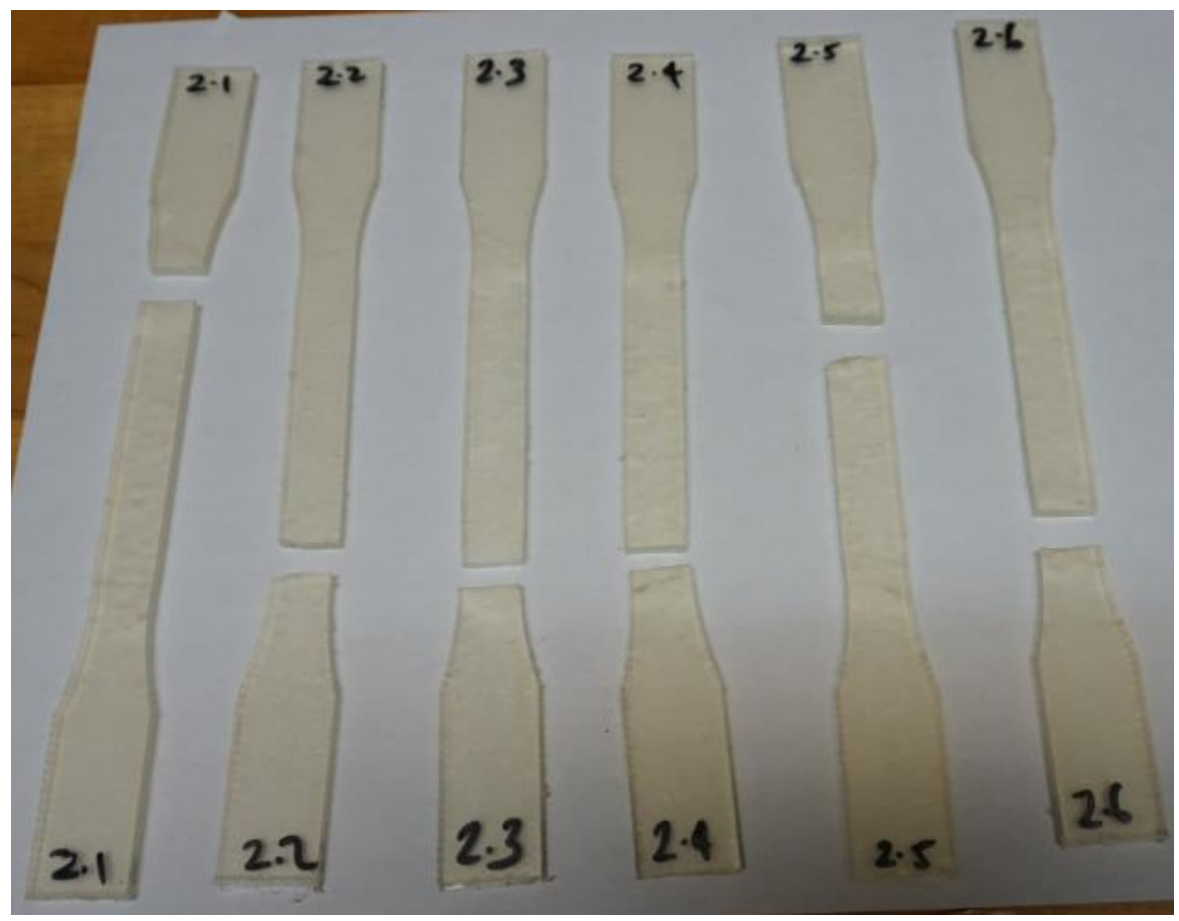

Figure 23: Fractured specimens of Batch-2 after tensile test.

\section{3 ВАТСН-3}

Tensile strength, tensile modulus and failure strain of all specimens of Batch-3 are listed in Table 10 with the resulting stress-strain graph shown in Figure 24 and a picture of coupons after failure are shown in Figure 25. Tensile strength of all specimen in this batch are in the same range with highest tensile strength tensile of 58.9 MPa and the lowest is 56.0 MPa for specimen \#3.2 and \#3.4 respectively. The highest tensile modulus of 3.4 GPa was obtained for \#3.6 and the lowest for \#3.4 with $2.99 \mathrm{GPa}$. Specimen \#3.6 and specimen \#3.1 had the highest failure strain of $2.03 \%$.

Table 10: Tensile strength, modulus and failure strain for Batch-3 specimens.

\begin{tabular}{|c|c|c|c|c|c|c|}
\hline Batch no: & Specimen \# & $\begin{array}{c}\text { Maximum } \\
\text { Force } \\
(\mathbf{l b s})\end{array}$ & $\begin{array}{c}\text { Area } \\
\left(\mathbf{i n}^{2}\right)\end{array}$ & $\begin{array}{c}\text { Strength } \\
(\mathbf{M P a})\end{array}$ & $\begin{array}{c}\text { Tensile } \\
\text { Modulus } \\
(\mathbf{G P a})\end{array}$ & $\begin{array}{c}\text { Failure } \\
\text { Strain } \\
(\boldsymbol{\%})\end{array}$ \\
\hline & 3.1 & 635.3 & 0.08 & 58.2 & 3.32 & 2.03 \\
& 3.2 & 670.9 & 0.08 & 58.9 & 3.39 & 2.15 \\
3 & 3.3 & 647.5 & 0.08 & 56.8 & 3.02 & 1.76 \\
& 3.4 & 545.5 & 0.07 & 56.1 & 2.99 & 2.02 \\
& 3.5 & 672.1 & 0.08 & 58.4 & 3.25 & 1.98 \\
& 3.6 & 598.5 & 0.07 & 58.6 & 3.40 & 2.03 \\
\hline
\end{tabular}




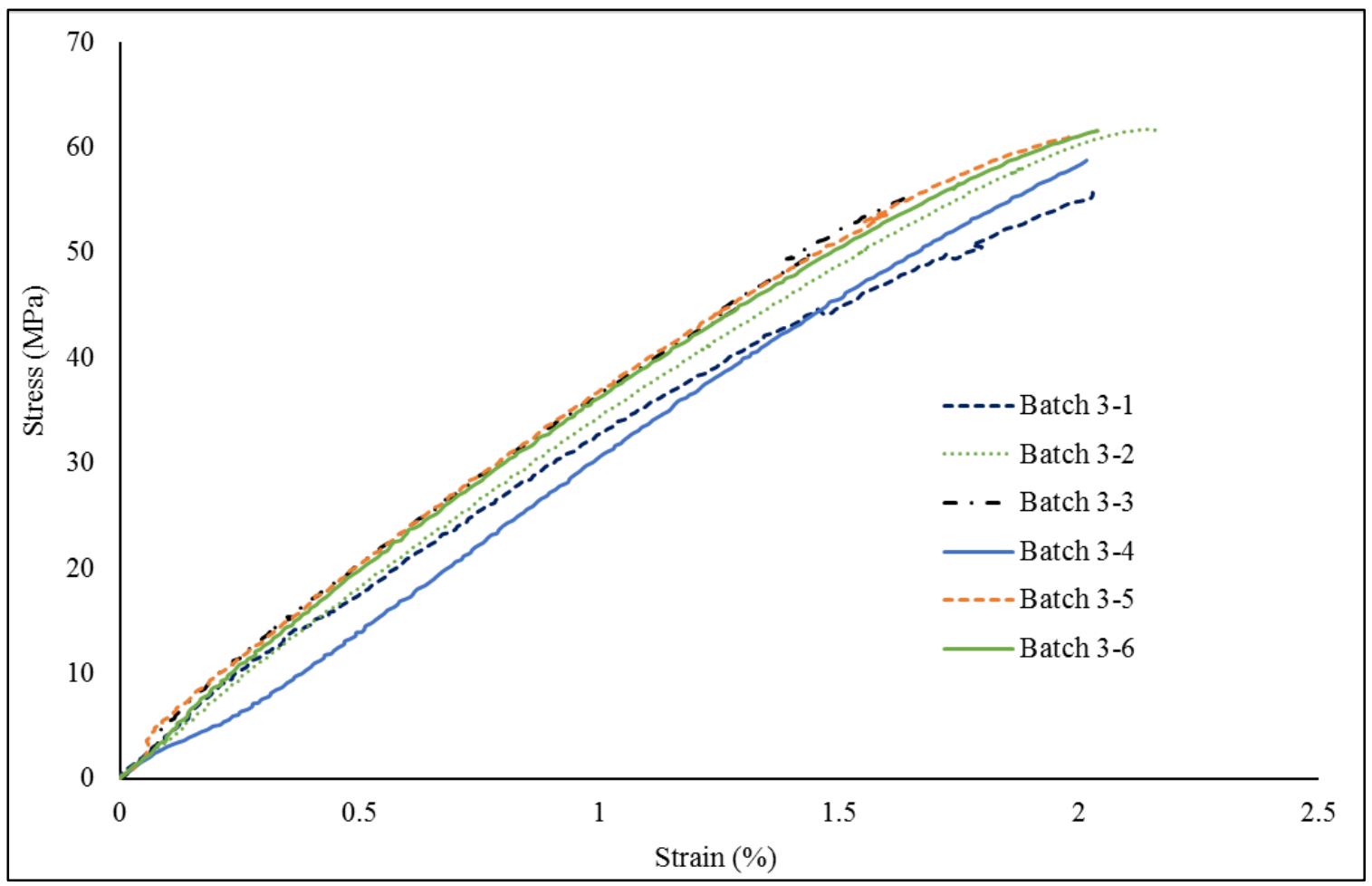

Figure 24: Stress-strain curve of Batch-3 specimens.

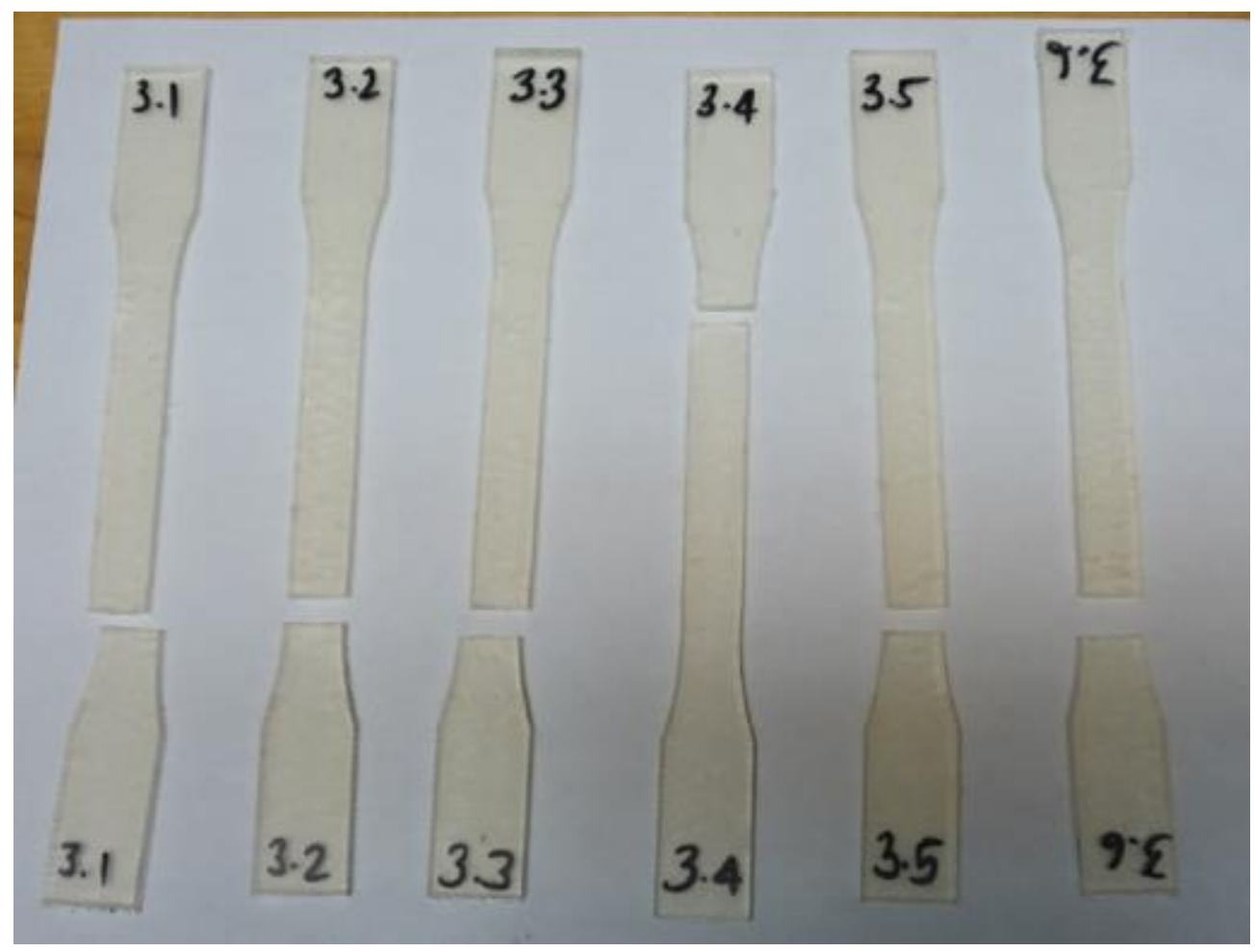

Figure 25: Fractured specimens of Batch-3 after tensile test. 


\section{4 ВАТСН-4}

Tensile strength, tensile modulus and failure strain of all specimens of Batch-4 are listed in Table 11 with the resulting stress-strain graph shown in Figure 26 and a picture of coupons after failure are shown in Figure 27. Tensile strength of all specimen in this batch are in the same range with highest tensile strength tensile of 59.6 MPa and the lowest is 57.0 MPa for specimen \#4.1 and \#4.5 respectively. The highest tensile modulus of $3.44 \mathrm{GPa}$ was obtained for specimen \#4.1 and the lowest for specimen \#4.3 with $2.98 \mathrm{GPa}$. Specimen \#4.2 with $3.36 \mathrm{GPa}$ tensile strength and a modulus of $3.44 \mathrm{GPa}$ had the highest failure strain of $2.16 \%$.

Table 11: Tensile strength, modulus and failure strain for Batch-4 specimens.

\begin{tabular}{|c|c|c|c|c|c|c|}
\hline Batch no: & Specimen \# & $\begin{array}{c}\text { Maximum } \\
\text { Force } \\
(\mathbf{l b s})\end{array}$ & $\begin{array}{c}\text { Area } \\
\left.\mathbf{( i n}^{2}\right)\end{array}$ & $\begin{array}{c}\text { Strength } \\
(\mathbf{M P a})\end{array}$ & $\begin{array}{c}\text { Tensile } \\
\text { Modulus } \\
(\mathbf{G P a})\end{array}$ & $\begin{array}{c}\text { Failure } \\
\text { Strain } \\
(\mathbf{\%})\end{array}$ \\
\hline \multirow{3}{*}{4} & 4.1 & 639.4 & 0.07 & 59.6 & 3.44 & 2.15 \\
& 4.2 & 669.4 & 0.08 & 58.8 & 3.36 & 2.16 \\
& 4.3 & 568.1 & 0.07 & 58.7 & 2.98 & 2.08 \\
& 4.4 & 663.5 & 0.08 & 59.5 & 3.29 & 2.11 \\
& 4.5 & 646.1 & 0.08 & 57.0 & 3.36 & 1.95 \\
\hline
\end{tabular}

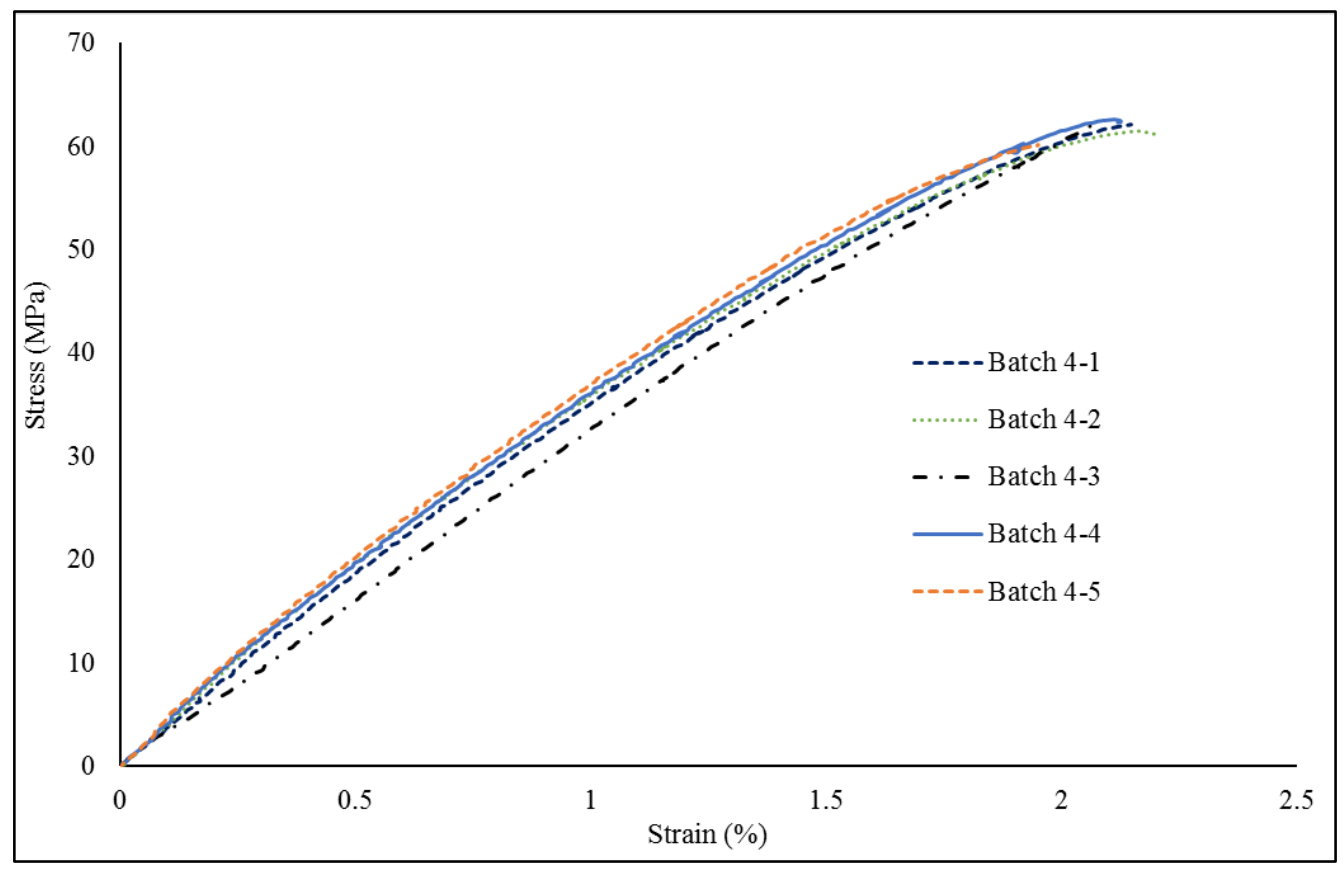

Figure 26: Stress-strain curve of Batch-4 specimens 


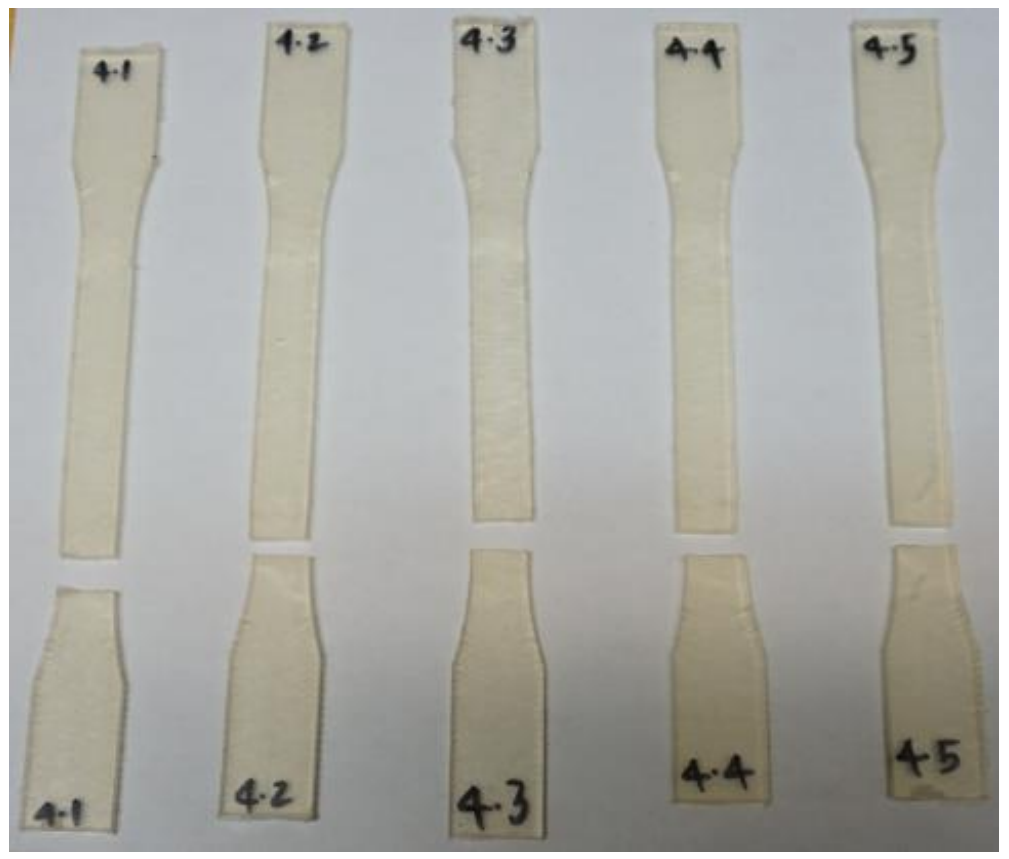

Figure 27: Fractured specimens of Batch-4 after tensile test.

\section{5 ВАТСН-5}

Tensile strength, tensile modulus and failure strain of all specimens of Batch-5 are listed in Table 12 with the resulting stress-strain graph shown in Figure 28 and a picture of coupons after failure are shown in Figure 29. Tensile strength of all specimen in this batch are in the same range with highest tensile strength tensile of 63.2 MPa and the lowest is 55.8 MPa for specimen \#5.2 and \#5.5 respectively. The highest tensile modulus of $3.48 \mathrm{GPa}$ was obtained for \#5.6 and the lowest for \#5.5 with 3.2 GPa. Specimen \#5.2 with highest tensile strength had highest failure strain of 2.19\%.

Table 12: Tensile strength, modulus and failure strain for Batch-5 specimens.

\begin{tabular}{|c|c|c|c|c|c|c|}
\hline Batch no: & Specimen \# & $\begin{array}{c}\text { Maximum } \\
\text { Force } \\
(\mathbf{l b s})\end{array}$ & $\begin{array}{c}\text { Area } \\
\left.\mathbf{( i n}^{2}\right)\end{array}$ & $\begin{array}{c}\text { Strength } \\
(\mathbf{M P a})\end{array}$ & $\begin{array}{c}\text { Tensile } \\
\text { Modulus } \\
(\mathbf{G P a})\end{array}$ & $\begin{array}{c}\text { Failure } \\
\text { Strain } \\
(\boldsymbol{\%})\end{array}$ \\
\hline & 5.2 & 650.0 & 0.07 & 63.2 & 3.27 & 2.19 \\
& 5.3 & 668.6 & 0.08 & 60.3 & 3.43 & 2.15 \\
5 & 5.4 & 655.8 & 0.08 & 57.8 & 3.29 & 2.00 \\
& 5.5 & 601.1 & 0.07 & 55.8 & 3.20 & 2.09 \\
& 5.6 & 664.1 & 0.07 & 62.4 & 3.48 & 2.12 \\
\hline
\end{tabular}




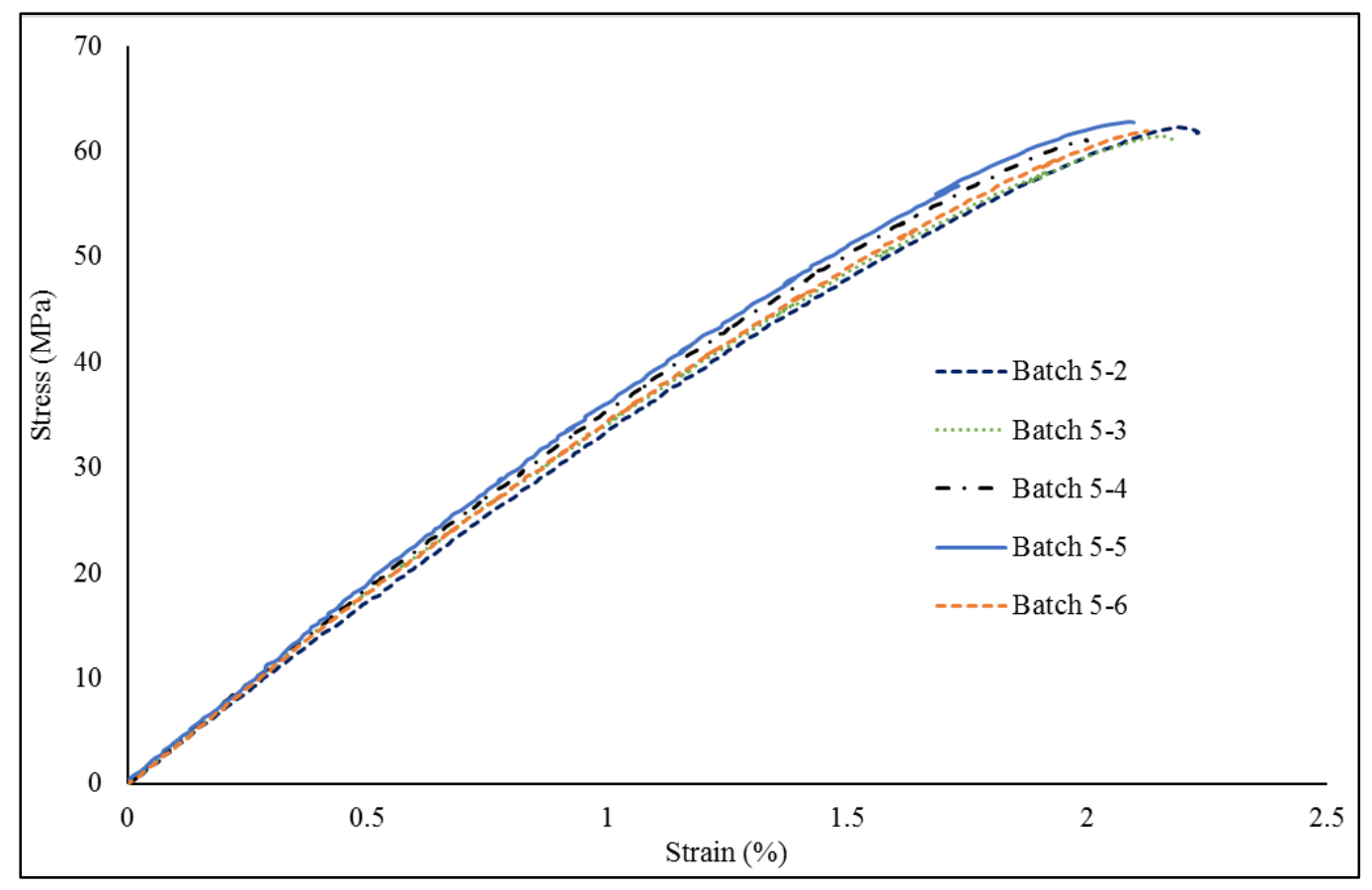

Figure 28: Stress-strain curve of Batch-5 specimens.

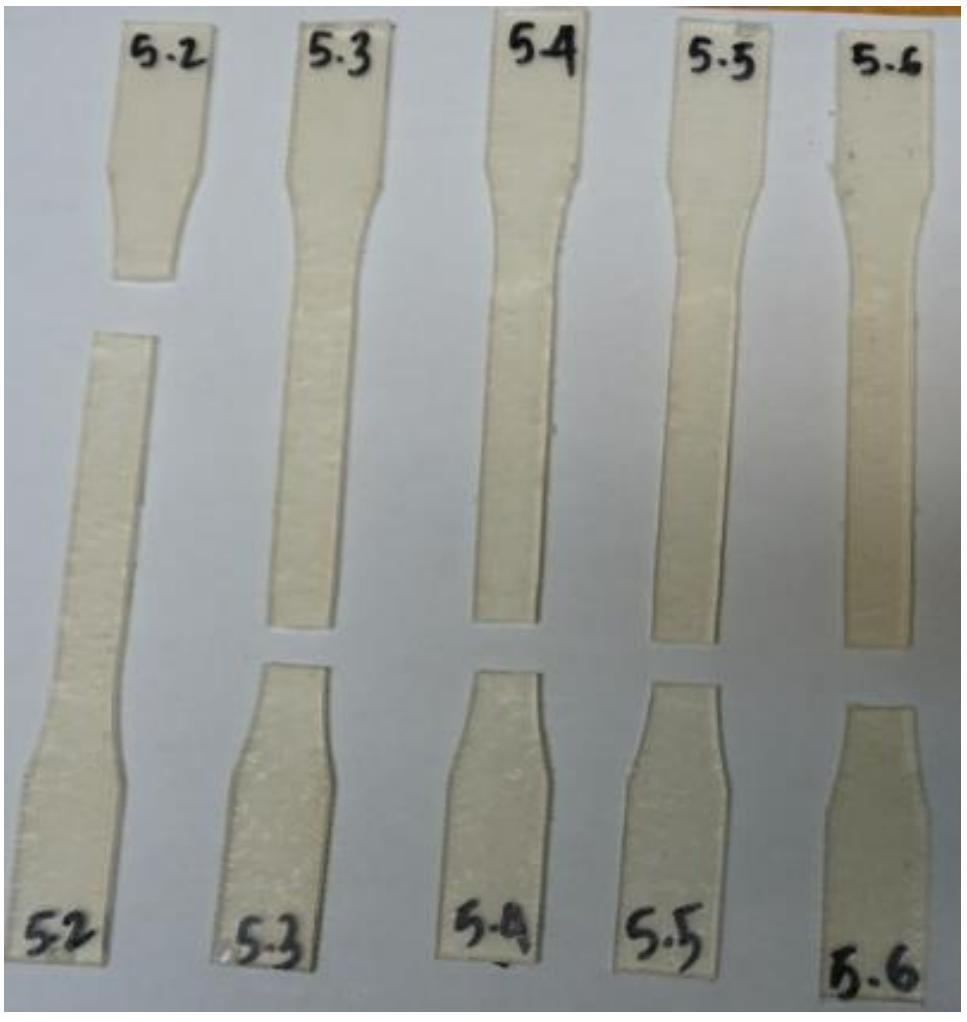

Figure 29: Fractured specimens of Batch-5 after tensile test. 


\section{6 ВАТСН-6}

Tensile strength, tensile modulus and failure strain of all specimens of Batch-6 are listed in Table 13 with the resulting stress-strain graph shown in Figure 30 and a picture of coupons after failure are shown in Figure 31. Tensile strength of all specimen in this batch are in the same range with highest tensile strength tensile of 60.2 MPa and the lowest is 56.7 MPa for specimen \#6.2 and \#6.3 respectively. The highest tensile modulus of $3.41 \mathrm{GPa}$ was obtained for \#6.1 and lowest for \#6.5 with $2.97 \mathrm{GPa}$. Specimen \#6.2 with highest tensile strength also had the highest failure strain of $2.20 \%$.

Table 13: Tensile strength, modulus and failure strain for Batch-6 specimens.

\begin{tabular}{|c|c|c|c|c|c|c|}
\hline Batch no: & Specimen \# & $\begin{array}{c}\text { Maximum } \\
\text { Force } \\
(\mathbf{l b s})\end{array}$ & $\begin{array}{c}\text { Area } \\
\left.\mathbf{( i n}^{2}\right)\end{array}$ & $\begin{array}{c}\text { Strength } \\
(\mathbf{M P a})\end{array}$ & $\begin{array}{c}\text { Tensile } \\
\text { Modulus } \\
(\mathbf{G P a})\end{array}$ & $\begin{array}{c}\text { Failure } \\
\text { Strain } \\
(\%)\end{array}$ \\
\hline & 6.1 & 676.6 & 0.08 & 59.9 & 3.41 & 2.13 \\
& 6.2 & 655.8 & 0.08 & 60.2 & 3.22 & 2.20 \\
6 & 6.3 & 647.8 & 0.08 & 56.7 & 3.09 & 1.95 \\
& 6.4 & 604.2 & 0.07 & 59.2 & 3.17 & 2.12 \\
& 6.5 & 566.3 & 0.07 & 58.6 & 2.97 & 2.11 \\
& 6.6 & 682.2 & 0.08 & 59.7 & 3.12 & 2.06 \\
\hline
\end{tabular}

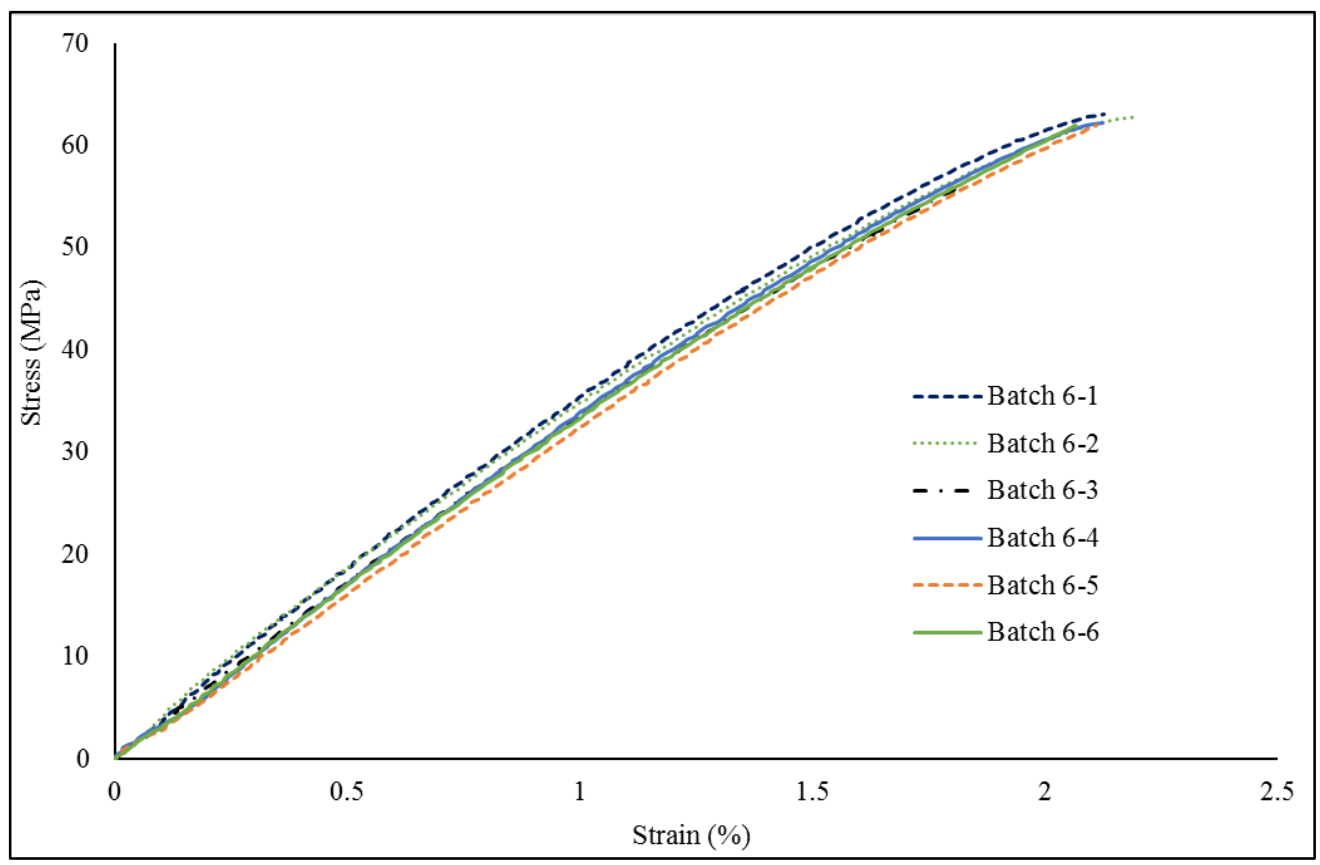

Figure 30: Stress-strain curve of Batch-6 specimens. 


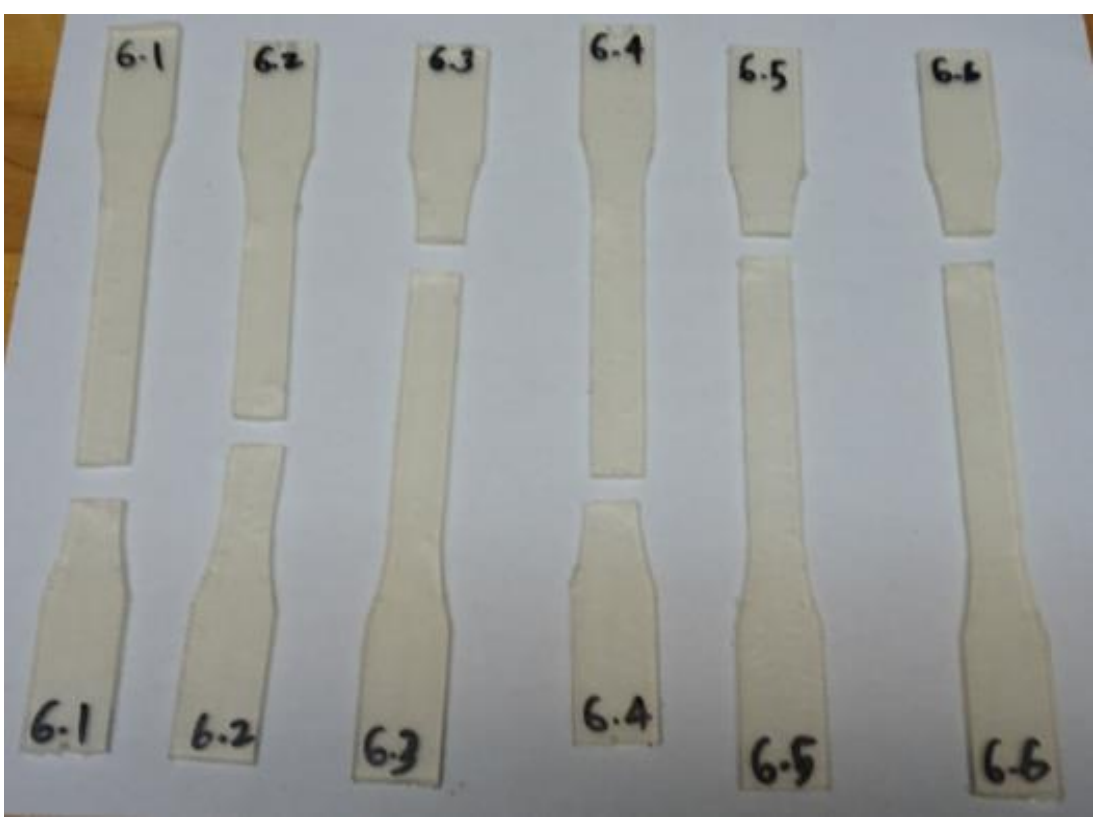

Figure 31: Fractured specimens of Batch-6 after tensile test.

\section{7 ВАТСН-7}

Tensile strength, tensile modulus and failure strain of all specimens of Batch-7 are listed in Table 14 with the resulting stress-strain graph shown in Figure 32 and a picture of coupons after failure are shown in Figure 33. Tensile strength of all specimen in this batch are in the same range with highest tensile strength tensile of $58.9 \mathrm{MPa}$ and the lowest is 55.0 $\mathrm{MPa}$ for specimen \#7.3 and \#7.1 respectively. The highest tensile modulus of $3.43 \mathrm{GPa}$ was obtained for \#7.5 and the lowest for \#7.1 with $2.78 \mathrm{GPa}$. Specimen \#7.5 with highest tensile strength had modulus of $3.43 \mathrm{GPa}$ and elongation of $2.02 \%$.

Table 14: Tensile strength, modulus and failure strain for Batch-7 specimens.

\begin{tabular}{|c|c|c|c|c|c|c|}
\hline Batch no: & Specimen \# & $\begin{array}{c}\text { Maximum } \\
\text { Force } \\
\text { (lbs) }\end{array}$ & $\begin{array}{c}\text { Area } \\
\text { (in2) }\end{array}$ & $\begin{array}{c}\text { Strength } \\
\text { (MPa) }\end{array}$ & $\begin{array}{c}\text { Tensile } \\
\text { Modulus } \\
(\mathbf{G P a})\end{array}$ & $\begin{array}{c}\text { Failure } \\
\text { Strain } \\
(\%)\end{array}$ \\
\hline \multirow{3}{*}{7} & 7.1 & 557.0 & 0.07 & 55.0 & 2.78 & 2.05 \\
& 7.2 & 652.0 & 0.08 & 56.9 & 3.24 & 1.95 \\
& 7.3 & 636.4 & 0.07 & 58.9 & 3.18 & 2.08 \\
& 7.4 & 598.1 & 0.07 & 58.9 & 3.32 & 2.08 \\
& 7.5 & 663.9 & 0.08 & 58.4 & 3.43 & 2.02 \\
\hline
\end{tabular}




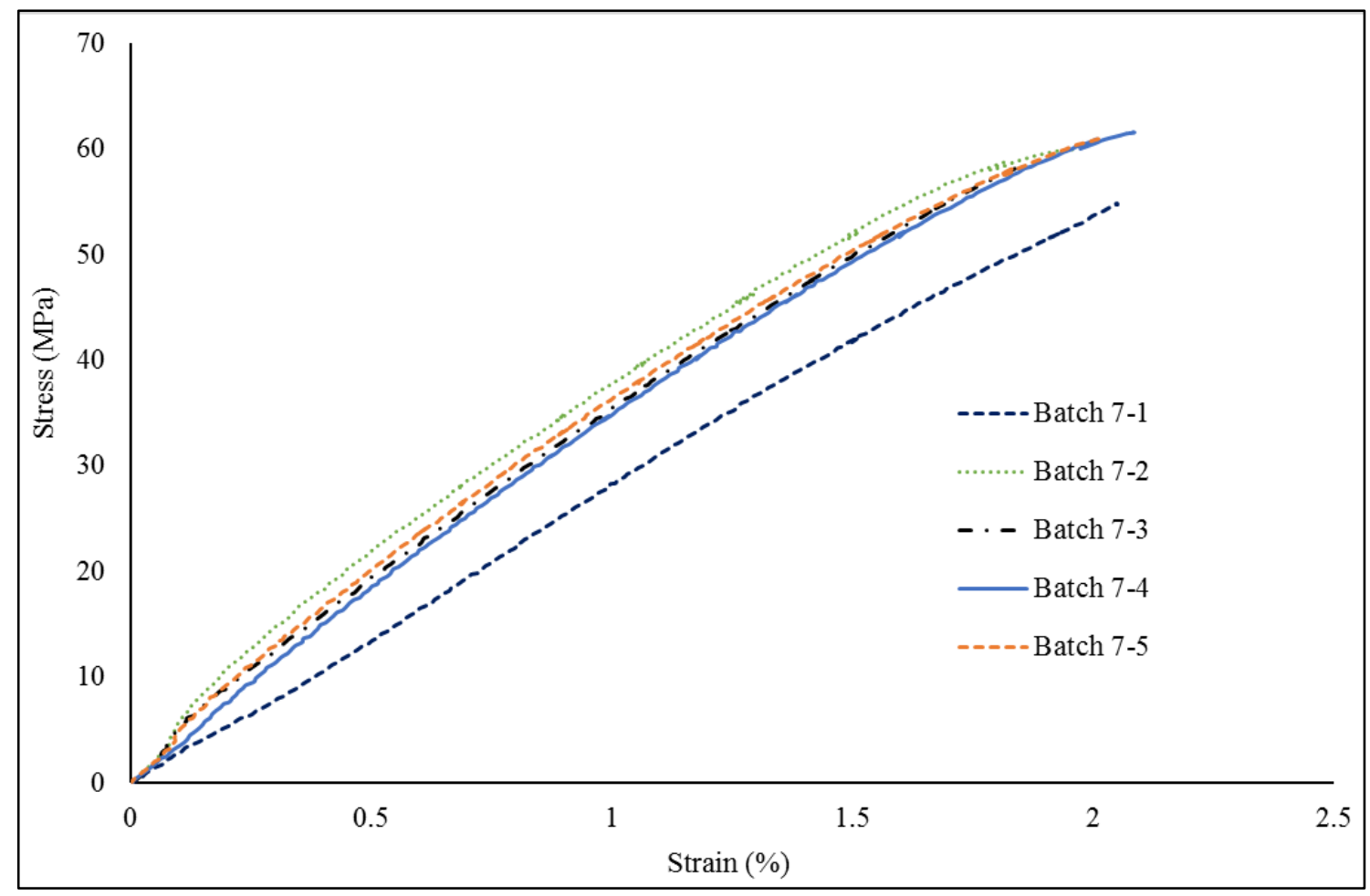

Figure 32: Stress-strain curve of Batch-7 specimens.

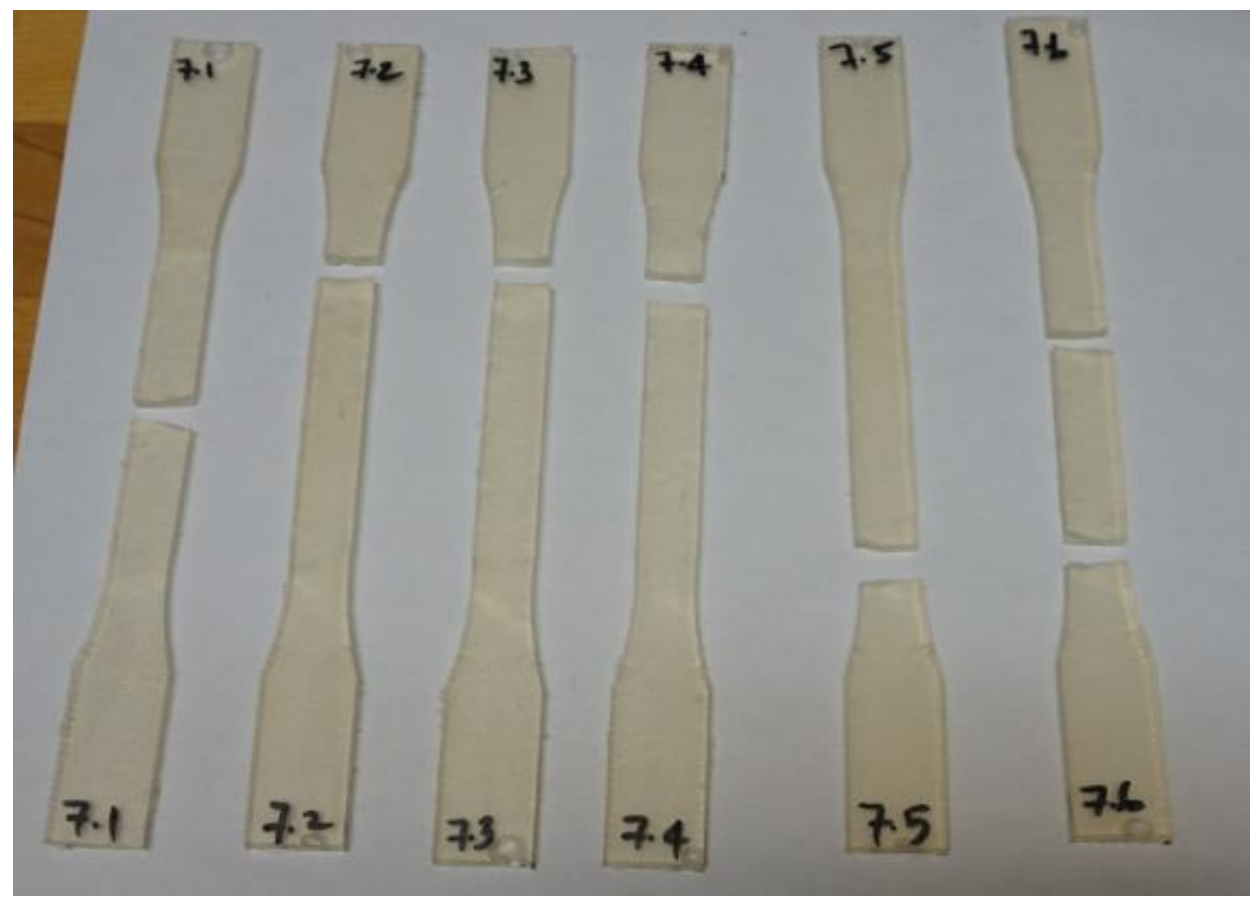

Figure 33: Fractured specimens of Batch-7 after tensile test. 


\section{8 ВАТСН-8}

Tensile strength, tensile modulus and failure strain of all specimens of Batch- 8 are listed in Table 15 with the resulting stress-strain graph shown in Figure 34 and a picture of coupons after failure are shown in Figure 35. Tensile strength of all specimen in this batch are in the same range with highest tensile strength tensile of $60.8 \mathrm{MPa}$ and the lowest is 59.5 MPa for specimen \#8.6 and \#8.5 respectively. The highest tensile modulus of $3.33 \mathrm{GPa}$ was obtained for \#8.1 and the lowest for \#8.5 with $2.94 \mathrm{GPa}$. Specimen \#8.5 with lowest tensile strength and tensile modulus had the highest failure strain of $2.31 \%$.

Table 15: Tensile strength, modulus and failure strain for Batch-8 specimens.

\begin{tabular}{|c|c|c|c|c|c|c|}
\hline Batch no: & Specimen \# & $\begin{array}{c}\text { Maximum } \\
\text { Force } \\
(\mathbf{l b s})\end{array}$ & $\begin{array}{c}\text { Area } \\
\left.\mathbf{( i n}^{2}\right)\end{array}$ & $\begin{array}{c}\text { Strength } \\
(\mathbf{M P a})\end{array}$ & $\begin{array}{c}\text { Tensile } \\
\text { Modulus } \\
(\mathbf{G P a})\end{array}$ & $\begin{array}{c}\text { Failure } \\
\text { Strain } \\
(\boldsymbol{\%})\end{array}$ \\
\hline & 8.1 & 681.5 & 0.08 & 59.9 & 3.33 & 2.19 \\
& 8.2 & 653.5 & 0.07 & 60.5 & 3.14 & 2.22 \\
8 & 8.3 & 614.8 & 0.07 & 60.4 & 3.27 & 2.22 \\
& 8.4 & 677.5 & 0.08 & 59.7 & 3.05 & 2.15 \\
& 8.5 & 568.5 & 0.07 & 59.5 & 2.94 & 2.31 \\
& 8.6 & 681.2 & 0.08 & 60.8 & 3.30 & 2.24 \\
\hline
\end{tabular}




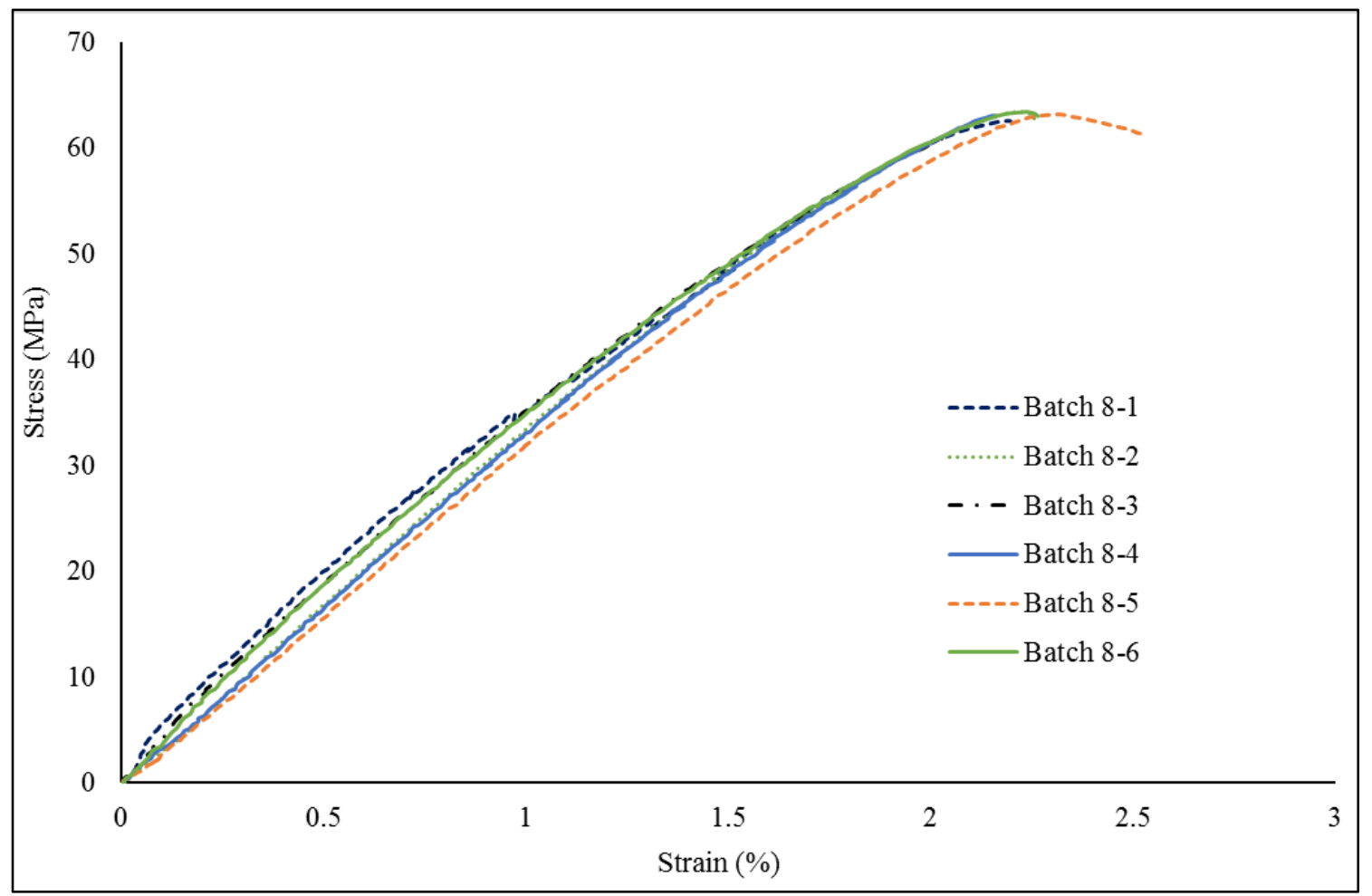

Figure 34: Stress-strain curve of Batch-8 specimens.

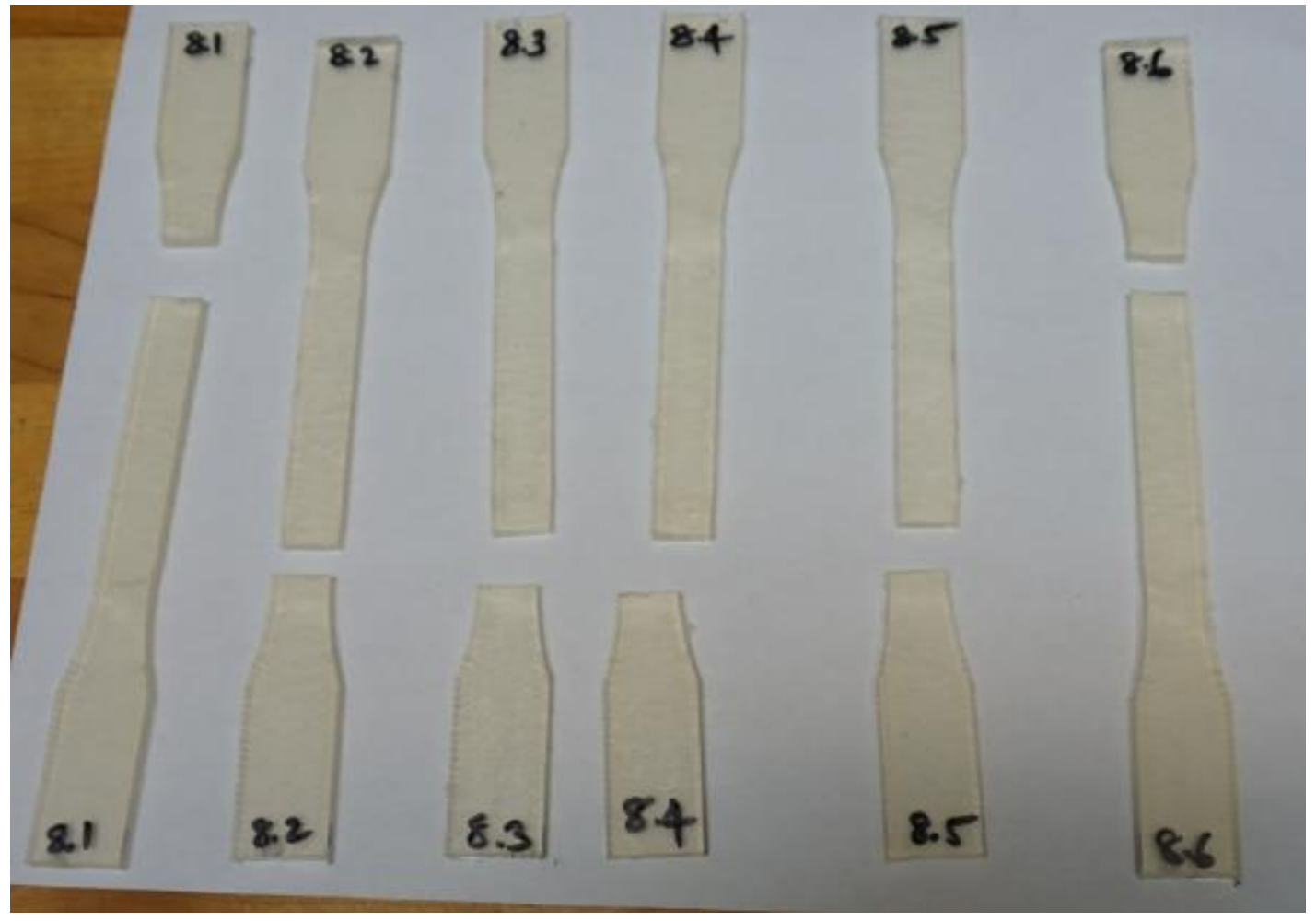

Figure 35: Fractured specimens of Batch-8 after tensile test. 


\section{9 ВАТСН-9}

Tensile strength, tensile modulus and failure strain of all specimens of Batch-9 are listed in Table 16 with the resulting stress-strain graph shown in Figure 36 and a picture of coupons after failure are shown in Figure 37. Tensile strength of all specimen in this batch are in the same range with highest tensile strength tensile of 60.1 MPa and the lowest is $56.5 \mathrm{MPa}$ for specimen \#9.5 and \#9.2 respectively. The highest tensile modulus of $3.46 \mathrm{GPa}$ is obtained for \#9.5 and the lowest for \#9.2 with $3.15 \mathrm{GPa}$. Specimen \#9.1 had the highest failure strain of $2.12 \%$.

Table 16: Tensile strength, modulus and failure strain for Batch-9 specimens.

\begin{tabular}{|c|c|c|c|c|c|c|}
\hline Batch no: & Specimen \# & $\begin{array}{c}\text { Maximum } \\
\text { Force } \\
(\mathbf{l b s})\end{array}$ & $\begin{array}{c}\text { Area } \\
\left(\mathbf{i n}^{2}\right)\end{array}$ & $\begin{array}{c}\text { Strength } \\
(\mathbf{M P a})\end{array}$ & $\begin{array}{c}\text { Tensile } \\
\text { Modulus } \\
(\mathbf{G P a})\end{array}$ & $\begin{array}{c}\text { Failure } \\
\text { Strain } \\
(\boldsymbol{\%})\end{array}$ \\
\hline \multirow{3}{*}{9} & 9.1 & 599.0 & 0.07 & 58.9 & 3.24 & 2.12 \\
& 9.2 & 647.7 & 0.08 & 56.5 & 3.15 & 1.98 \\
& 9.3 & 674.1 & 0.08 & 59.2 & 3.32 & 2.06 \\
& 9.5 & 646.8 & 0.07 & 60.1 & 3.46 & 1.84 \\
& 9.6 & 674.7 & 0.08 & 60.0 & 3.26 & 2.08 \\
\hline
\end{tabular}

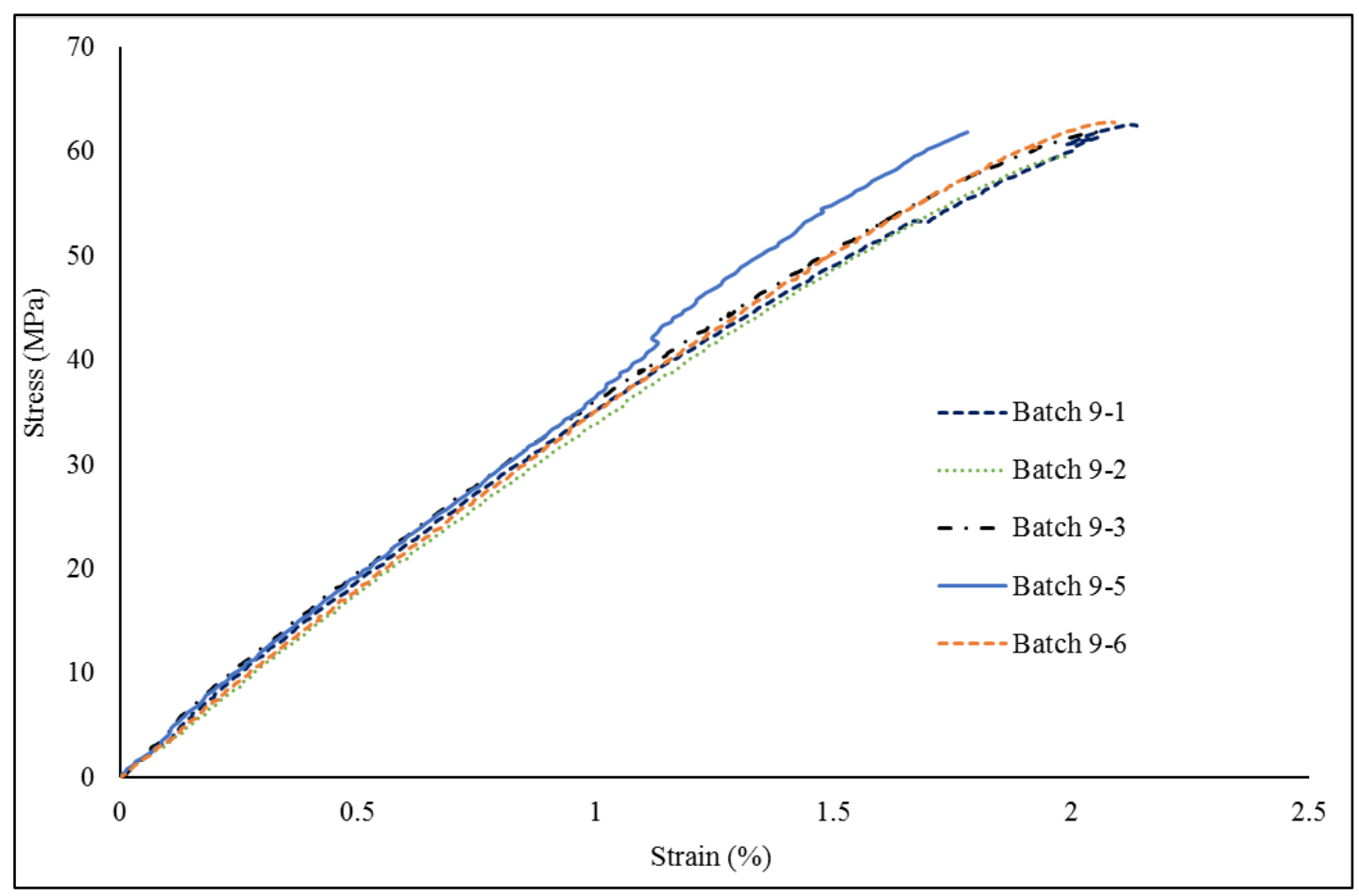

Figure 36: Stress-strain curve of Batch-9 specimens. 


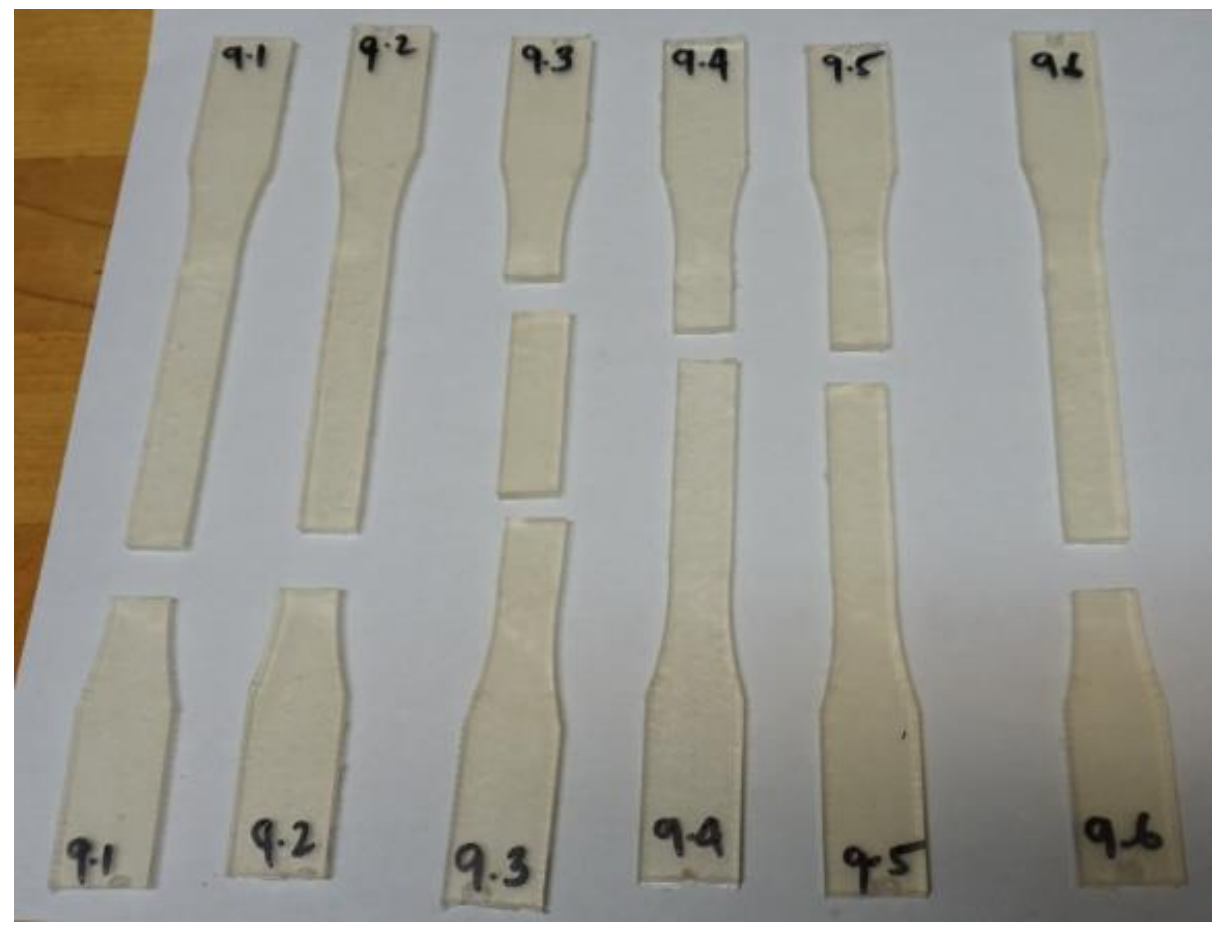

Figure 37: Fractured specimens of Batch-9 after tensile test.

To determine which combination of processing temperature, processing pressure and dwell time produced the best PLA specimen, a comparison of results from different batches is shown table. For each batch the mean tensile strength, tensile modulus and strain failure is tabulated with their respective coefficient of variance.

Table 17: Averaged Tensile strength, Modulus and failure strain values for all batches.

\begin{tabular}{|c|c|c|c|c|c|c|}
\hline Batch no. & $\begin{array}{c}\text { Mean } \\
\text { Strength } \\
(\mathbf{M P a})\end{array}$ & $\begin{array}{c}\mathbf{C V} \\
(\boldsymbol{\%})\end{array}$ & $\begin{array}{c}\text { Mean } \\
\text { Modulus } \\
\mathbf{( G P a )}\end{array}$ & $\begin{array}{c}\mathbf{C V} \\
(\boldsymbol{\%})\end{array}$ & $\begin{array}{c}\text { Mean Failure } \\
\text { Strain } \\
(\boldsymbol{\%})\end{array}$ & $\begin{array}{c}\mathbf{C V} \\
(\boldsymbol{\%})\end{array}$ \\
\hline 1 & 58.4 & 1.4 & 3.22 & 3.9 & 1.99 & 2.9 \\
\hline 2 & 59.5 & 2.9 & 3.27 & 5.3 & 2.15 & 5.0 \\
\hline 3 & 57.8 & 2.0 & 3.23 & 5.6 & 1.99 & 6.3 \\
\hline 4 & 58.7 & 1.7 & 3.28 & 5.5 & 2.09 & 4.1 \\
\hline 5 & 59.9 & 5.2 & 3.33 & 3.5 & 2.11 & 3.5 \\
\hline 6 & 59.0 & 2.1 & 3.16 & 4.6 & 2.10 & 3.9 \\
\hline 7 & 57.6 & 2.9 & 3.19 & 7.8 & 2.04 & 2.6 \\
\hline 8 & 60.1 & 0.8 & 3.17 & 4.8 & 2.22 & 2.3 \\
\hline 9 & 59.0 & 2.5 & 3.29 & 3.4 & 2.02 & 5.4 \\
\hline
\end{tabular}


Graphical comparison of mechanical properties obtained for different batches obtained from the test is showed in Figure 38-Figure 40.

In case of tensile strength, Batch-8 has the highest tensile strength of $60.1 \mathrm{MPa}$, closely followed by Batch-5, 2, and 6 with 59.9 MPa, 59.5 MPa, 59.0 MPa respectively. The lowest tensile strength of 57.60 MPa is obtained for Batch-7.

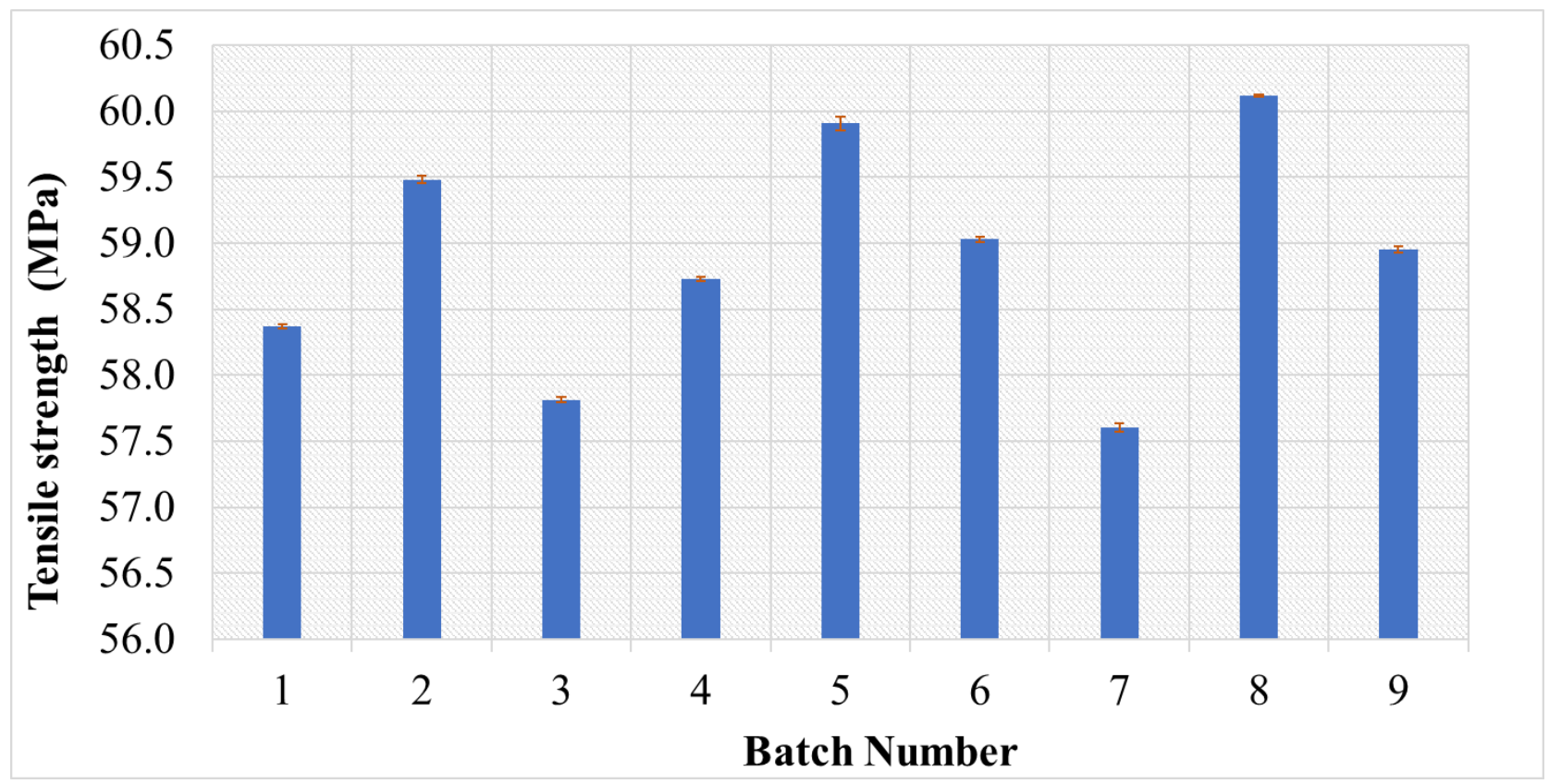

Figure 38: Comparison of averaged tensile strength values for all batches.

Graphical comparison of tensile modulus, Figure 39, of all different batches shows Batch-5 has the highest tensile modulus of 3.33 GPa. 3.29, 3.28, and 3.27 GPa are the next best values obtained for Batch-9, 4, and 2. Batch-8 recorded only 3.17 GPa, although it had the highest tensile strength value. 


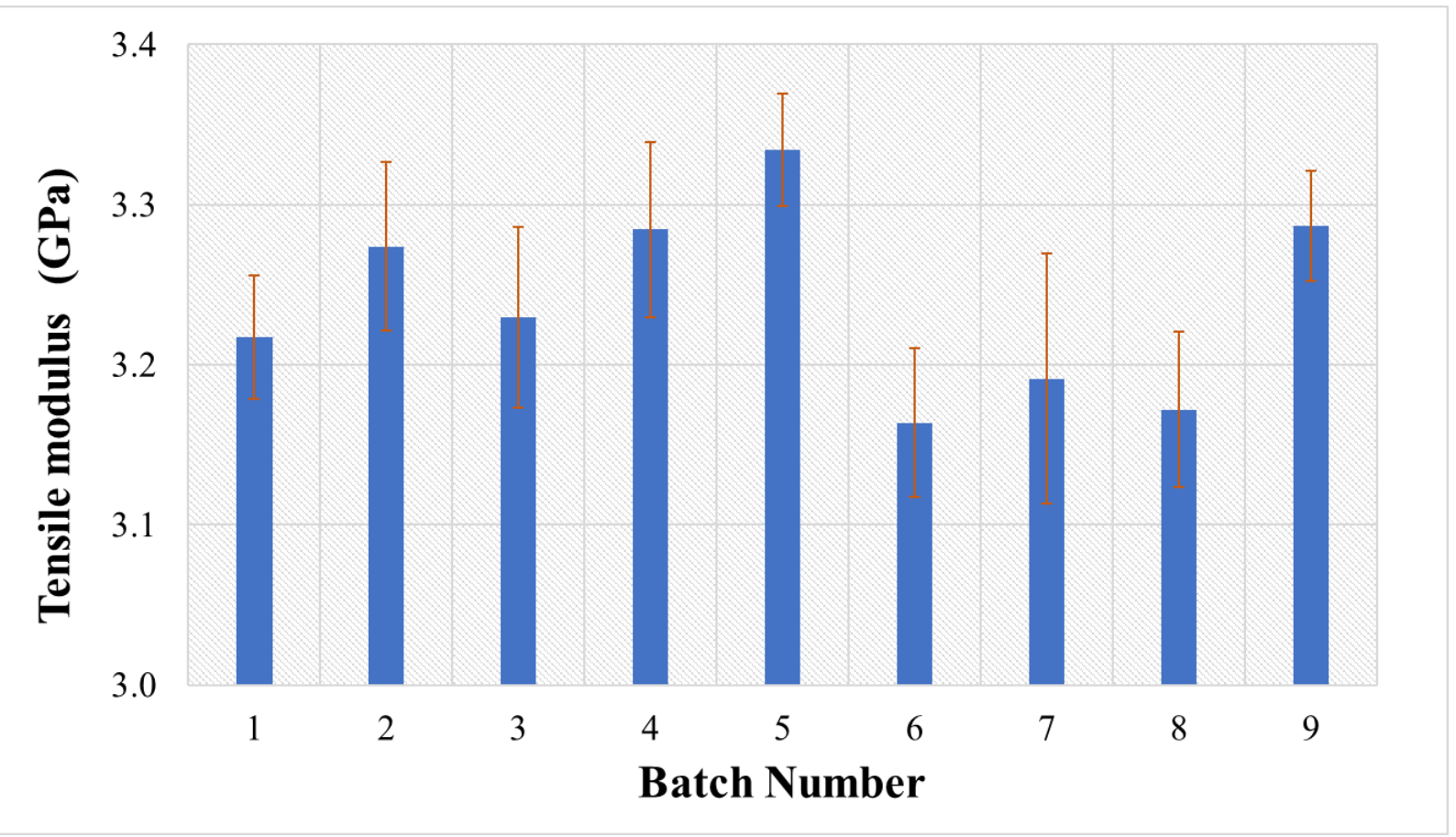

Figure 39: Averaged tensile modulus comparison of all batches.

As shown in Figure 40, percent elongation of Batch-8 is the highest at 2.22\%. The lowest elongation is for Batch-1 and Batch-3 of 1.99\%. Batch-5 with highest tensile modulus has an elongation of $2.11 \%$.

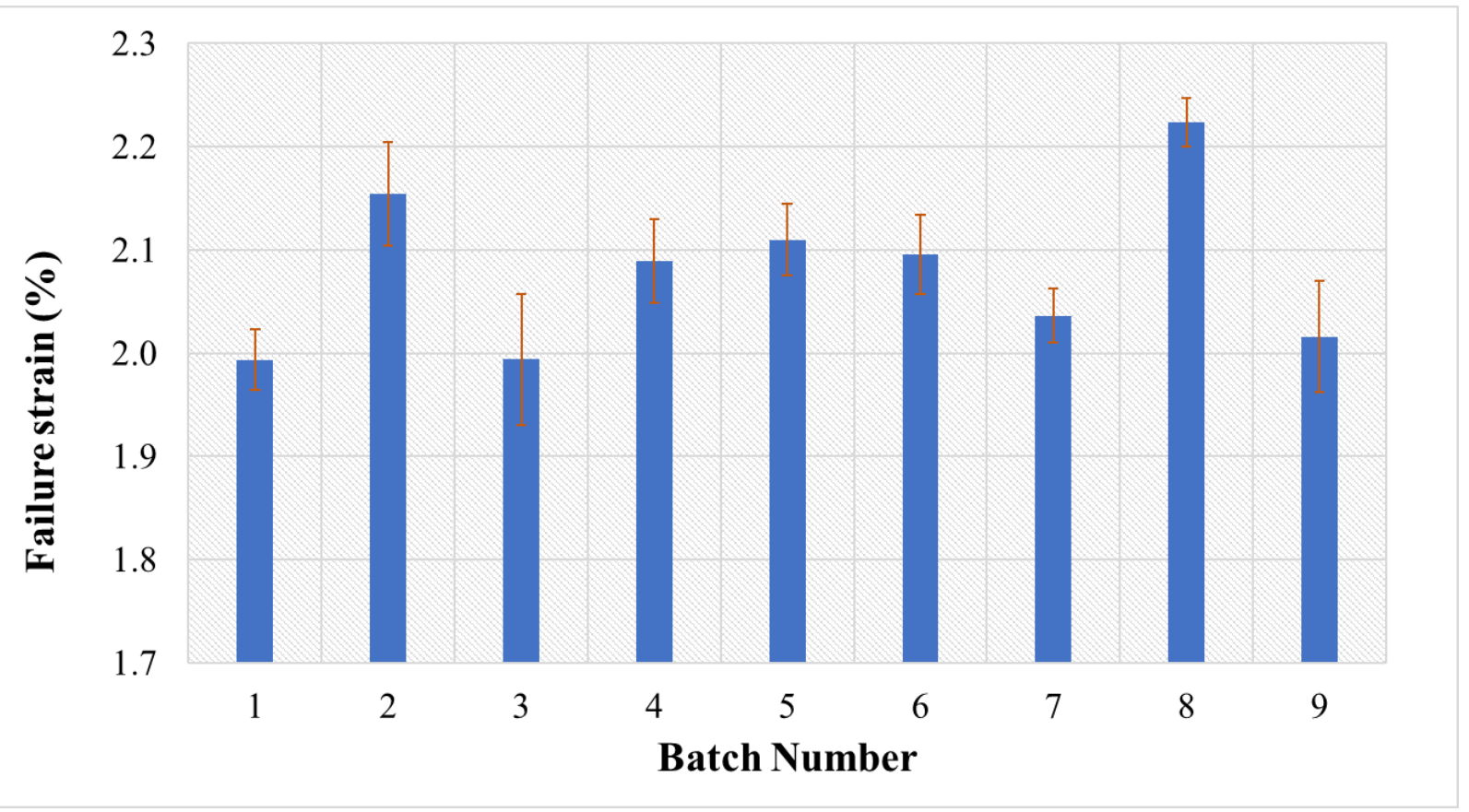

Figure 40: Failure strain of all batches. 


\section{DISCUSSION}

For the ease of analyzing the test results to understand the effect of processing parameters (temperature, pressure and dwell time) on the mechanical properties, batches with same processing temperatures are grouped together. Batch-1, 2, and 3 with temperature $150^{\circ} \mathrm{C}$ form group $\mathrm{A}$, Batches-4, 5, and 6 are group B, and Batches- 7,8 , and 9 with $170{ }^{\circ} \mathrm{C}$ form group C.

In group A, among batches 1,2 and 3 that were processed at a temperature of $150^{\circ} \mathrm{C}, \mathrm{Batch}-2$ has recorded the highest tensile strength and tensile modulus of 59.5 MPa and $3.27 \mathrm{GPa}$, respectively. Since temperature for all three batches were same, it is evident that higher strength property is attributed to varying Pressure and dwell times. Similarly, in group B Batch-5 has the highest tensile strength and modulus of 59.9 MPa and $3.33 \mathrm{GPa}$, respectively and in group C Batch-8 with 60.12 $\mathrm{MPa}$ and $3.17 \mathrm{GPa}$. One thing that is common with batches 2, 5 and 8 is the processing pressure of 2.5 MPa. This indicates that the optimal pressure to process this PLA pellets is $2.5 \mathrm{MPa}$.

Now to analyze the optimal temperature, properties of batches 2,5 , and 8 processed at 150, 160, and $170^{\circ} \mathrm{C}$, respectively can be compared. Batch- 8 has the highest tensile strength of $60.1 \mathrm{MPa}$ followed by Batch-5 with $59.9 \mathrm{MPa}$ and Batch-2 with $59.5 \mathrm{MPa}$. However, Batch-5 and 2 have better tensile modulus of 3.33 and $3.27 \mathrm{GPa}$ than Batch-8 with 3.17GPa. Since tensile modulus of Batch-5 is $4.8 \%$ more than Batch- 8 but tensile strength of 5 is only $0.3 \%$ less, we can decide that Batch-5 has better mechanical properties than Batch- 8 . Similarly, tensile modulus of Batch- 2 is about 3\% more than that of Batch- 8 but only $1 \%$ less in tensile strength of Batch- 8 . Therefore, processing temperatures of 160 and $150{ }^{\circ} \mathrm{C}$ is better than $170{ }^{\circ} \mathrm{C}$. This is in agreement with literature surveys [4] that suggests ideal temperature for compression molding PLA is usually ten degrees more than the melting temperature of PLA pellets, in this case $160{ }^{\circ} \mathrm{C}$ is the ideal temperature.

A weighted average property comparison of all the batches performed and presented in Table 18 to find optimum compression molding process parameters. Weighted average property is calculated by considering the tensile strength and tensile modulus of each batch manufactured by compression molding. 


$$
\text { Weighted Average Property }(W A P)=\frac{X 1}{\max X}+\frac{Y 1}{\max Y}
$$

Where,

$\mathrm{X}_{1 \text { is }}$ tensile strength of a batch.

$\mathrm{Y}_{1}$ is tensile modulus of a batch.

Max $\mathrm{X}$ is the maximum value of tensile strength among all batches.

Max $\mathrm{Y}$ is the maximum value of tensile modulus among all batches.

Table 18: Weighted average property (WAP) comparison of all batches.

\begin{tabular}{|c|c|}
\hline Batch \#: & WAP \\
\hline 1 & 1.94 \\
\hline 2 & 1.97 \\
\hline 3 & 1.93 \\
\hline 4 & 1.96 \\
\hline 5 & 2.00 \\
\hline 6 & 1.93 \\
\hline 7 & 1.92 \\
\hline 8 & 1.95 \\
\hline 9 & 1.97 \\
\hline
\end{tabular}

Based on the weighted average property comparison, Batch-5 has the optimum properties among all the compression molded batches. Properties of Batch-5 are compared in the following sections with properties of $3 \mathrm{D}$ printed specimens. 


\section{COMPARISON WITH 3D PRINTED PLA SPECIMENS}

All 3D specimens have similar dimensions but depending on the amount of deposition, the weight of each specimen vary. The maximum weight is $10.4 \mathrm{~g}$ and the lowest $9.45 \mathrm{~g}$ for specimen \#3 and specimen \#1 respectively. Results of tensile test performed as per ASTM D638-14 and fractured specimens are shown in Figure 41. As shown in Table 19, among the FFF 3D printed specimens the highest tensile strength and tensile modulus value is obtained for specimen \#3, 64.7 MPa and 3.31GPa respectively. This is apparent considering the weight of specimen \#3 which is highest among batch. The lowest strength and modulus values are obtained for specimen \#1 with $55.7 \mathrm{MPa}$ and $2.99 \mathrm{GPa}$ respectively. In terms of failure strain, Specimen \#3 clearly has the highest failure strain of $2.54 \%$ and the lowest is obtained for specimen \#6 with $2.01 \%$.

Table 19: Tensile strength, tensile modulus and failure strain of 3D printed specimens.

\begin{tabular}{|l|c|c|c|c|c|c|c|}
\hline Batch no: & $\begin{array}{c}\text { Specimen } \\
\#\end{array}$ & $\begin{array}{c}\text { Weight } \\
\text { (g) }\end{array}$ & $\begin{array}{c}\text { Maximum } \\
\text { Force } \\
\text { (lbs) }\end{array}$ & $\begin{array}{c}\text { Area } \\
\text { (in2) }\end{array}$ & $\begin{array}{c}\text { Strength } \\
\text { (MPa) }\end{array}$ & $\begin{array}{c}\text { Tensile } \\
\text { Modulus } \\
\text { (GPa) }\end{array}$ & $\begin{array}{c}\text { Failure } \\
\text { Strain } \\
(\boldsymbol{\%})\end{array}$ \\
\hline & 1 & 9.45 & 553.1 & 0.07 & 55.7 & 2.99 & 2.25 \\
& 2 & 9.89 & 574.3 & 0.07 & 58.0 & 3.18 & 2.20 \\
3D Printed & 3 & 10.8 & 655.2 & 0.07 & 64.7 & 3.31 & 2.54 \\
specimens & 4 & 10.4 & 619.3 & 0.07 & 62.1 & 3.26 & 2.34 \\
& 5 & 10.6 & 614.1 & 0.07 & 61.0 & 3.29 & 2.17 \\
& 6 & 10.2 & 580.1 & 0.07 & 58.2 & 3.28 & 2.01 \\
\hline
\end{tabular}




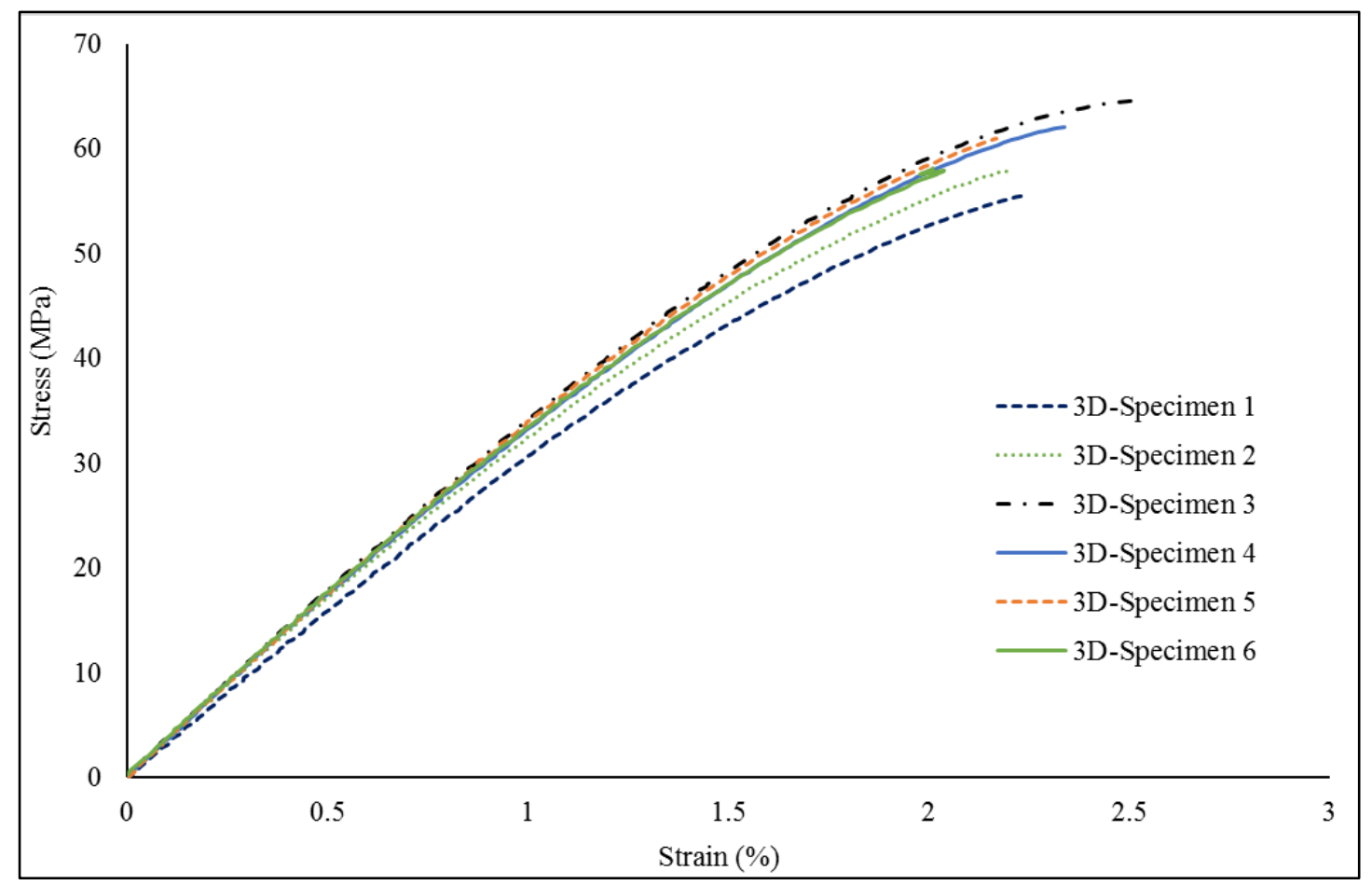

Figure 41: Stress- Strain graph of 3D printed specimens.

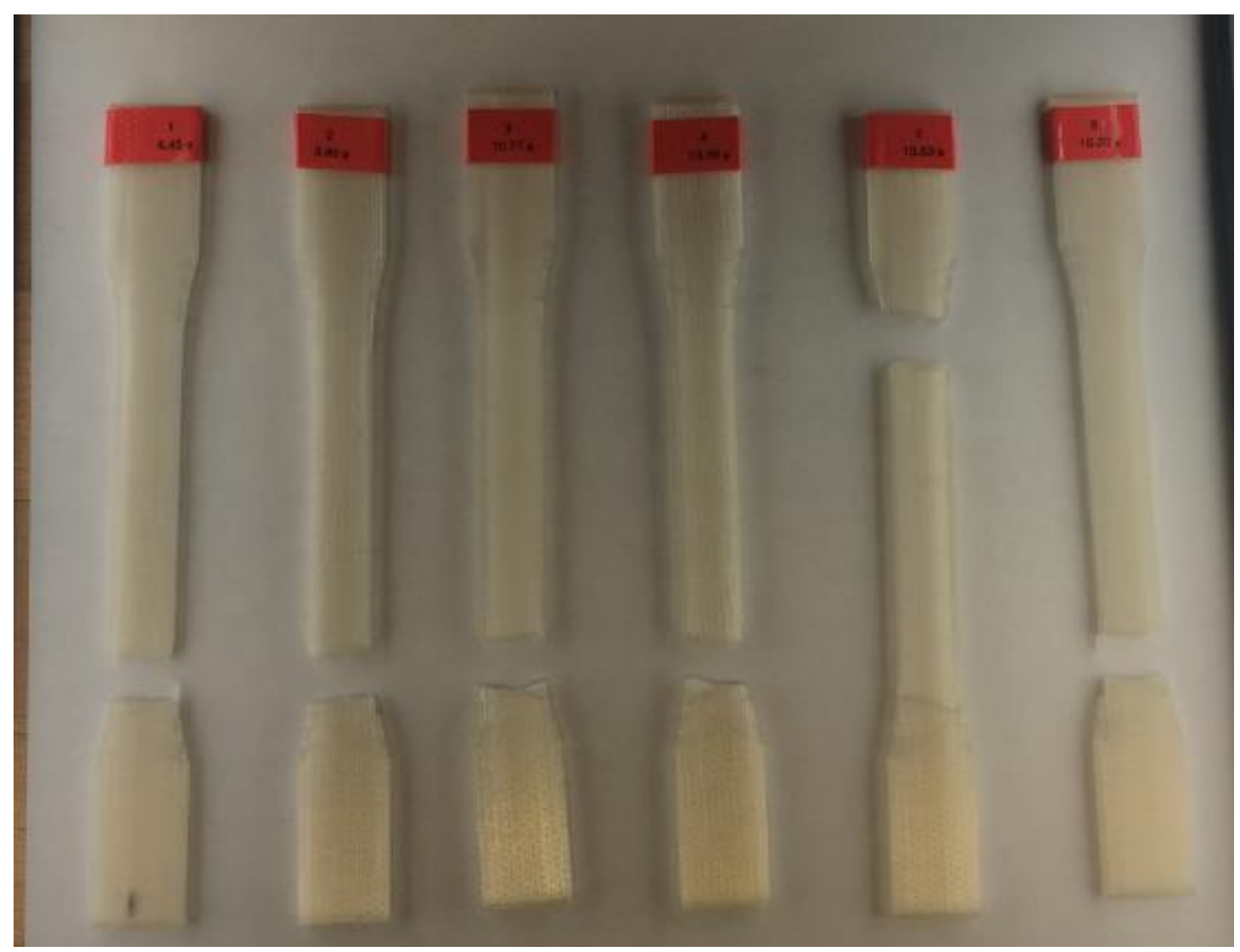

Figure 42: Fractured 3D printed specimens. 
To understand the influence of manufacturing process on the strength properties, a comparison of compression molded PLA results against the mean value of the 3D printed specimens are considered and shown in Table 20.

Table 20: Comparison of Compression molded PLA specimen and 3D printed PLA specimen.

\begin{tabular}{|c|c|c|c|}
\hline Batch no: & $\begin{array}{c}\text { Mean Strength } \\
\text { (MPa) }\end{array}$ & $\begin{array}{c}\text { Mean Modulus } \\
(\mathbf{G P a})\end{array}$ & $\begin{array}{c}\text { Mean Failure } \\
\text { Strain } \\
(\boldsymbol{\%})\end{array}$ \\
\hline 1 & 58.37 & 3.22 & 1.99 \\
\hline 2 & 59.48 & 3.27 & 2.15 \\
\hline 3 & 57.81 & 3.23 & 1.99 \\
\hline 4 & 58.73 & 3.28 & 2.09 \\
\hline 5 & 59.91 & 3.33 & 2.11 \\
\hline 6 & 59.03 & 3.16 & 2.10 \\
\hline 7 & 57.60 & 3.19 & 2.04 \\
\hline 8 & 60.12 & 3.17 & 2.22 \\
\hline 9 & 58.95 & 3.29 & 2.02 \\
\hline 3D-Specimen & 59.94 & 3.22 & 2.25 \\
\hline
\end{tabular}

Tensile properties obtained for 3D printed specimen is comparable and very close to the values obtained for compression molded PLA specimens. Comparing Batch-5, the best properties among the compression molded, with 3D specimen its evident that the tensile strength of both specimens are same but tensile modulus of compression molded specimen is slightly higher at $3.33 \mathrm{GPa}$ against 3.22GPa of 3D printed specimen.

A Stress- strain curve comparison of both compression mold (Batch-5) and 3D printed specimens is shown Figure 43. 


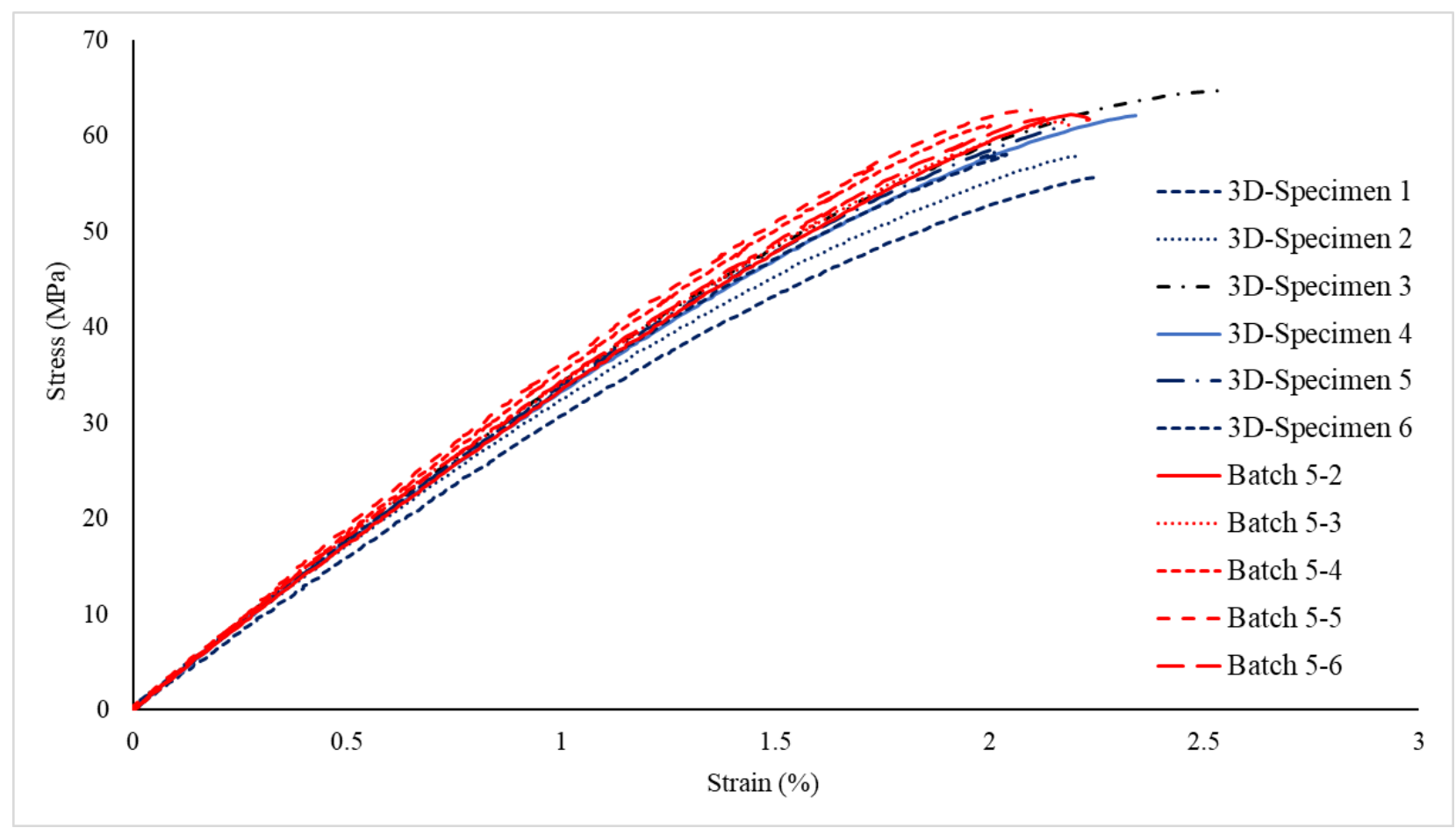

Figure 43 : Stress- Strain curve comparison of CM specimen vs 3D specimen.

The stress- strain curve suggest a similar trend between the compression molded PLA specimens and 3D printed PLA specimens. The rate of strain for both specimens are similar; however, the area under compression molded specimens is marginally higher suggesting capable of absorbing more energy before failure than the 3D printed specimens.

In order to assess better, a weighted average of properties is performed to determine which specimens have better properties. An average of tensile strength and tensile modulus of all compression molded batches and 3D printed specimen as shown in Table 21. The Batch-5 has a weighted average of 2 (1.997) against 1.96 weighted average of 3D printed specimen. 
Table 21: Weighted average property comparison of compression molded specimens and 3D specimen.

\begin{tabular}{|c|c|}
\hline Batch no: & WAP \\
\hline 1 & 1.94 \\
\hline 2 & 1.97 \\
\hline 3 & 1.93 \\
\hline 4 & 1.96 \\
\hline 5 & 2.00 \\
\hline 6 & 1.93 \\
\hline 7 & 1.92 \\
\hline 8 & 1.95 \\
\hline 9 & 1.97 \\
\hline 3D printed Specimen & 1.96 \\
\hline
\end{tabular}

The mechanical properties obtained for 3D printing are very similar to the properties obtained for compression molding. However, compression molded specimens have marginally better stiffness than 3D printed specimen. In compression molding the PLA material is processed under pressure and temperature which allows consolidation of molecules in to a compact part with better stiffness. The cooling cycle and process cycle are processed under pressure unlike in 3D printing methodology. This clearly can be attributed to this advantage for obtaining better samples. 


\section{CONCLUSION AND FUTURE SCOPE}

This study compared the performance of compression-molded and 3D printed PLA. First, the compression molding manufacturing parameters were optimized to obtain higher mechanical properties. This study investigates the effect of each parameters including processing temperature, processing pressure, and dwell times on strength properties of a pure PLA parts as per ASTM D638-14. The entire study is conducted on a total of 54 specimens manufactured in a set of 9 batches for various combination of process parameters. To understand the effect of each manufacturing process, a comprehensive comparison of the properties of the compression-molded PLA with 3D printed PLA specimens is presented.

The effects of the processing parameters on the strength of the material have been analyzed and optimum conditions for manufacturing PLA samples with better tensile properties are determined. For various temperature conditions, the optimum processing pressure is $2.5 \mathrm{MPa}$. Furthermore, the optimum processing temperature is $160^{\circ} \mathrm{C}$, which is ten degrees more than the melting temperature of PLA $\left(150{ }^{\circ} \mathrm{C}\right)$ and is in good agreement with the literature survey [4]. On the comparison of tensile strength and tensile modulus of all 54 specimens manufactured in 9 batches, the optimum values were $59.9 \mathrm{MPa}$ and $3.33 \mathrm{GPa}$, respectively.

Optimized 3D printing process parameters were found in previous studies and were used here to manufacture specimens per ASTM D638. The tensile strength and tensile modulus values of 3D specimen obtained (i.e., 59.9 MPa and 3.2 GPa) are very close and comparable to those obtained using compression molded specimens. In terms of tensile strength, both processes yield very similar results, but specimens that are compression molded had a slightly better stiffness and this could be attributed to the fact that in compression molding molecules are incorporated better under pressure and temperature than in 3D printing. Although, not very significant improvement yet its agreeable to conclude that compression molding thermoplastics provides slightly better strength properties. However, with right manufacturing parameters 3D printing is capable of competing with conventional methods and produce components with equivalent properties. 
Future Scope of study:

- The optimized parameters for compression molding a pure PLA sample for enhanced tensile strength is established. Determining other mechanical properties like flexural property, impact strength, etc. can be studied.

- Addition of fibers: With pure PLA, compression molding has a slight advantage over 3D printing, but with addition of fibers this might change. A future study can work on the new optimized processing parameters for the addition of fibers.

- Moisture absorption in PLA: A study to understand how moisture impacts strength of a PLA specimen and whether there is a strength deterioration can also be considered.

- A comparison between compression molded and 3D printed specimens in terms of moisture absorption and its impact on the mechanical performance. 


\section{REFERENCES}

1. Gupta, A. P., \& Kumar, V. (2007). New emerging trends in synthetic biodegradable polymers-Polylactide: A critique. European polymer journal, 43(10), 4053-4074.

2. Shibata, M., Oyamada, S., Kobayashi, S., \& Yaginuma, D. (2004). Mechanical properties and biodegradability of green composites based on biodegradable polyesters and lyocell fabric. Journal of Applied Polymer Science, 92(6), 3857-3863.

3. Srebrenkoska, V., \& Bogoeva-Gaceva, G. (2014). Preparation and recycling of polymer ecocomposites. Macedonian Journal of Chemistry and Chemical Engineering, 28, 99-109

4. Kakar.A, Jayamani.E, Heng, S.K., Bakri, M. K., Hamdan, S. (2015). Optimization of Hot Press Compression Molding and Fabrication of Poly Lactic Acid (PLA) Luffa Biocomposites for Biomedical Applications. Australian Journal of Basic and Applied Sciences, 9(8), 105112.

5. Rubio-López, A., Olmedo, A., Díaz-Álvarez, A., \& Santiuste, C. (2015). Manufacture of compression moulded PLA based biocomposites: A parametric study. Composite Structures, $131,995-1000$.

6. Memon, A., \& Nakai, A. (2013). Fabrication and mechanical properties of jute spun yarn/PLA unidirection composite by compression molding. Energy Procedia, 34, 830-838.

7. Blok, L. G., Longana, M. L., Yu, H., \& Woods, B. K. S. (2018). An investigation into 3D printing of fibre reinforced thermoplastic composites. Additive Manufacturing, 22, 176-186.

8. Shikamoto, N., Wongsriraksa, P., Ohtani, A., Wei, L. Y., \& Nakai, A. (2008). Processing and mechanical properties of biodegradable composites. In The 9th International Conference on Flow Processes in Composite Materials.

9. Graupner, N., Herrmann, A. S., \& Müssig, J. (2009). Natural and man-made cellulose fibrereinforced poly (lactic acid)(PLA) composites: An overview about mechanical characteristics and application areas. Composites Part A: Applied Science and Manufacturing, 40(6-7), 810-821

10. Graupner, N., Ziegmann, G., Wilde, F., Beckmann, F., \& Müssig, J. (2016). Procedural influences on compression and injection moulded cellulose fibre-reinforced polylactide (PLA) composites: Influence of fibre loading, fibre length, fibre orientation and voids. Composites Part A: Applied Science and Manufacturing, 81, 158-171. 
11. Graupner, N., \& Müssig, J. (2011). A comparison of the mechanical characteristics of kenaf and lyocell fibre reinforced poly (lactic acid)(PLA) and poly (3-hydroxybutyrate)(PHB) composites. Composites Part A: Applied Science and Manufacturing, 42(12), 2010-2019.

12. Carneiro, O. S., Silva, A. F., \& Gomes, R. (2015). Fused deposition modeling with polypropylene. Materials \& Design, 83, 768-776.

13. Bajpai, P. K., Singh, I., \& Madaan, J. (2014). Development and characterization of PLAbased green composites: A review. Journal of Thermoplastic Composite Materials, 27(1), 52-81.

14. Raj, S. A., Muthukumaran, E., \& Jayakrishna, K. (2018). A Case Study of 3D Printed PLA and Its Mechanical Properties. Materials Today: Proceedings, 5(5), 11219-11226.

15. Ochi, S. (2008). Mechanical properties of kenaf fibers and kenaf/PLA composites. Mechanics of materials, 40(4-5), 446-452.

16. Tao, Y. U., Yan, L. I., \& Jie, R. E. N. (2009). Preparation and properties of short natural fiber reinforced poly (lactic acid) composites. Transactions of Nonferrous Metals Society of China, 19, 651-655.

17. UMassAmherst. (2018, Dec 17). UMassAmherst. Retrieved from Introduction to Taguchi Method: http://www.ecs.umass.edu/mie/labs/mda/fea/sankar/chap2.html

18. Fayazbakhsh, K., Movahedi, M., \& Kalman, J. (2019). The impact of defects on tensile properties of 3D printed parts manufactured by fused filament fabrication. Materials Today Communications, 18, 140-148.

19. ASTM D4703-16 Standard Practice for Compression Molding Thermoplastic Materials into Test Specimens, Plaques, or Sheets.,1-17.

20. ASTM D638-14 Standard Test Method for Tensile Properties of Plastics.,1-13. 